\title{
Interstellar Mapping and Acceleration Probe (IMAP): A New NASA Mission
}

D.J. McComas' ${ }^{1}$ - E.R. Christian ${ }^{2}$ - N.A. Schwadron ${ }^{3}$ N. Fox ${ }^{4}$ J. Westlake ${ }^{4}$. F. Allegrini ${ }^{5,6}$ - D.N. Baker ${ }^{7}$ D. Biesecker ${ }^{8}$ - M. Bzowski ${ }^{9}$. G. Clark $^{4}$ - C.M.S. Cohen ${ }^{10}$. I. Cohen ${ }^{4}$ - M.A. Dayeh ${ }^{5,6} \cdot$ R. Decker $^{4}$ - G.A. de Nolfo ${ }^{2}$ - M.I. Desai ${ }^{5,6} \cdot$ R.W. Ebert ${ }^{5}$. H.A. Elliott ${ }^{5}$ - H. Fahr ${ }^{11}$ - P.C. Frisch ${ }^{12}$ - H.O. Funsten ${ }^{13}$ - S.A. Fuselier F, $^{5,6}$. A. Galli ${ }^{14}$. A.B. Galvin ${ }^{3}$. J. Giacalone ${ }^{15}$ - M. Gkioulidou ${ }^{4}$. F. Guo ${ }^{13}$ - M. Horanyi ${ }^{7}$ - P. Isenberg $^{3}$. P. Janzen ${ }^{16}$ - L.M. Kistler ${ }^{3}$ K. Korreck ${ }^{17}$ - M.A. Kubiak ${ }^{9}$ H. Kucharek ${ }^{3}$. B.A. Larsen ${ }^{13}$ - R.A. Leske ${ }^{10}$ • N. Lugaz ${ }^{3}$ J. Luhmann ${ }^{18}$ - W. Matthaeus ${ }^{19}$. D. Mitchell ${ }^{4}$ - E. Moebius ${ }^{3}$ - K. Ogasawara ${ }^{5}$ - D.B. Reisenfeld ${ }^{13}$ - J.D. Richardson ${ }^{20}$. C.T. Russell ${ }^{21}$ - J.M. Sokót ${ }^{9}$ - H.E. Spence ${ }^{3}$ - R. Skoug ${ }^{13}$ • Z. Sternovsky ${ }^{7}$. P. Swaczyna ${ }^{1}$ - J.R. Szalay ${ }^{1}$ - M. Tokumaru ${ }^{22}$ - M.E. Wiedenbeck ${ }^{23}$ • P. Wurz ${ }^{14}$. G.P. Zank ${ }^{24,25}$ - E.J. Zirnstein ${ }^{1}$

Received: 11 September 2018 / Accepted: 5 October 2018 / Published online: 22 October 2018 (C) The Author(s) 2018

Abstract The Interstellar Mapping and Acceleration Probe (IMAP) is a revolutionary mission that simultaneously investigates two of the most important overarching issues in

Electronic supplementary material The online version of this article (https://doi.org/10.1007/s11214-018-0550-1) contains supplementary material, which is available to authorized users.

D.J. McComas

dmccomas@princeton.edu

1 Department of Astrophysical Sciences, Princeton University, Princeton, NJ 08544, USA

2 Heliophysics Science Division, NASA Goddard Space Flight Center, Greenbelt, MD 20771, USA

3 Space Science Center, University of New Hampshire, Durham, NH 03824, USA

4 Johns Hopkins University Applied Physics Laboratory, Laurel, MD 20723, USA

5 Southwest Research Institute, San Antonio, TX 78228, USA

6 Department of Physics and Astronomy, University of Texas at San Antonio, San Antonio, TX 78249, USA

7 Laboratory for Atmospheric and Space Physics, University of Colorado Boulder, Boulder, CO 80303, USA

8 Space Weather Prediction Center, National Oceanic and Atmospheric Administration, Boulder, CO 80305, USA

9 Space Research Centre, Polish Academy of Sciences (CBK PAN), Bartycka 18A, Warsaw, Poland

10 California Institute of Technology, Pasadena, CA 91125, USA

11 Argelander Institut für Astronomie, Universität Bonn, Bonn, Germany

12 Department of Astronomy and Astrophysics, University of Chicago, Chicago, IL 60637, USA

13 Los Alamos National Laboratory, Los Alamos, NM 87545, USA 
Heliophysics today: the acceleration of energetic particles and interaction of the solar wind with the local interstellar medium. While seemingly disparate, these are intimately coupled because particles accelerated in the inner heliosphere play critical roles in the outer heliospheric interaction. Selected by NASA in 2018, IMAP is planned to launch in 2024. The IMAP spacecraft is a simple sun-pointed spinner in orbit about the Sun-Earth L1 point. IMAP's ten instruments provide a complete and synergistic set of observations to simultaneously dissect the particle injection and acceleration processes at $1 \mathrm{AU}$ while remotely probing the global heliospheric interaction and its response to particle populations generated by these processes. In situ at 1 AU, IMAP provides detailed observations of solar wind electrons and ions; suprathermal, pickup, and energetic ions; and the interplanetary magnetic field. For the outer heliosphere interaction, IMAP provides advanced global observations of the remote plasma and energetic ions over a broad energy range via energetic neutral atom imaging, and precise observations of interstellar neutral atoms penetrating the heliosphere. Complementary observations of interstellar dust and the ultraviolet glow of interstellar neutrals further deepen the physical understanding from IMAP. IMAP also continuously broadcasts vital real-time space weather observations. Finally, IMAP engages the broader Heliophysics community through a variety of innovative opportunities. This paper summarizes the IMAP mission at the start of Phase A development.

Keywords Heliosphere · Interstellar medium · IBEX · IMAP · ENA · Energetic particle

$\begin{array}{ll}\text { Abbreviations } \\ \text { ACE } & \text { Advanced Composition Explorer (mission) } \\ \text { ACR } & \text { Anomalous cosmic ray } \\ \text { AMPTE } & \text { Active Magnetospheric Particle Tracer Explorers (mission) } \\ \text { AO } & \text { Announcement of Opportunity } \\ \text { APD } & \text { Avalanche Photodiode } \\ \text { AU } & \text { Astronomical Unit } \\ \text { CDA } & \text { Cosmic Dust Analyzer (on Cassini) }\end{array}$

14 Physics Institute, University of Bern, Bern, 3012, Switzerland

15 Department of Planetary Sciences, University of Arizona, Tucson, AZ 85721, USA

16 Department of Physics and Astronomy, University of Montana, Missoula, MT 59812, USA

17 Smithsonian Astrophysical Observatory, Harvard-Smithsonian Center for Astrophysics, Cambridge, MA 02138, USA

18 Space Sciences Laboratory, University of California, Berkeley, CA 94720, USA

19 Department of Physics and Astronomy, University of Delaware, Newark, DE 19716, USA

20 Kavli Center for Astrophysics and Space Science, Massachusetts Institute of Technology, Cambridge, MA 02139, USA

21 Department of Earth, Planetary, and Space Sciences, University of California, Los Angeles, CA 90095, USA

22 Institute for Space-Earth Environmental Research, Nagoya University, Nagoya 464-8601, Japan

23 Jet Propulsion Laboratory, California Institute of Technology, Pasadena, CA 91109, USA

24 Department of Space Science, University of Alabama in Huntsville, Huntsville, AL 35899, USA

25 Center for Space Plasma and Aeronomic Research (CSPAR), University of Alabama in Huntsville, Huntsville, AL 35805, USA 


$\begin{array}{ll}\text { CDF } & \text { Computable Document Format } \\ \text { CIR } & \text { Corotating interaction region } \\ \text { CME } & \text { Coronal mass ejection }\end{array}$

CoDICE Compact Dual Ion Composition Experiment (on IMAP)

CS Conversion surface

DSCOVR Deep Space Climate Observatory

DSN Deep Space Network

ENA Energetic neutral atom

EP Energetic particle

ESA Electrostatic analyzer

EUV Extreme ultraviolet

FITS Flexible Image Transport System

FOV Field-of-view

FWHM Full-width at half-maximum

GCR Galactic cosmic ray

GEM Genesis Electron Monitor (on Genesis)

GF Geometric factor

GLOWS Global Solar Wind Structure (on IMAP)

HFL Heliophysics Future Leaders

HIT High-energy Ion Telescope (on IMAP)

HOPE Helium, Oxygen, Proton, and Electron (on Van Allen Probes)

HSO Heliophysics System Observatory

HV High voltage

I-ALiRT IMAP Active Link for Real-Time

IBEX Interstellar Boundary Explorer (mission)

ICME Interplanetary coronal mass ejection

IDEX Interstellar Dust Experiment (on IMAP)

IMAP Interstellar Mapping and Acceleration Probe

IMC IMAP Magnetospheric CubeSat

INCA Ion and Neutral Camera (on Cassini)

ISD Interstellar dust

ISEE International Sun-Earth Explorer (mission)

ISN Interstellar neutral

JENI Jupiter Energetic Neutral Imager (on JUICE)

L1 Earth-Sun Lagrange Point 1

LET Low Energy Telescope (on STEREO)

LISM Local interstellar medium

MAG Fluxgate magnetometer (on IMAP)

MCP Microchannel plate

MMS Magnetospheric Multiscale (mission)

MOC Mission Operations Center

NASA National Aeronautics and Space Administration (agency)

NH New Horizons (mission)

NOAA National Oceanic and Atmospheric Administration (agency)

PA Pivot angle

PUI Pickup ion

RTSW Real-Time Space Weather

SEP Solar energetic particle

SNR Signal-to-noise ratio 


$\begin{array}{ll}\text { SOC } & \text { Science Operations Center } \\ \text { SPDF } & \text { Space Physics Data Facility } \\ \text { SSD } & \text { Solid state detector } \\ \text { ST } & \text { Suprathermal Ions } \\ \text { STEREO } & \text { Solar Terrestrial Relations Observatory (mission) } \\ \text { STP } & \text { Solar Terrestrial Probe } \\ \text { SWAP } & \text { Solar Wind Around Pluto (on NH) } \\ \text { SWAPI } & \text { Solar Wind and Pickup Ion (on IMAP) } \\ \text { SWE } & \text { Solar Wind Electron (on IMAP) } \\ \text { SWEPAM } & \text { Solar Wind Electron, Proton, and Alpha Monitor (on ACE) } \\ \text { SWOOPS } & \text { Solar Wind Observations Over the Poles of the Sun (on Ulysses) } \\ \text { SWPC } & \text { Space Weather Prediction Center (at NOAA) } \\ \text { TDO } & \text { Technology Demonstration Opportunity } \\ \text { TOF } & \text { Time-of-flight } \\ \text { TWINS } & \text { Two Wide-Angle Imaging Neutral-Atom Spectrometers (mission) } \\ \text { UV } & \text { Ultraviolet } \\ \text { VA } & \text { Variable aperture } \\ \text { VDF } & \text { Velocity distribution function }\end{array}$

\section{Introduction and Overview}

The Interstellar Mapping and Acceleration Probe (IMAP) mission had its genesis in the National Academy of Science's 2012 Heliophysics Decadal Survey (National Research Council 2013), where the Decadal Survey team merged two of the proposed mission concepts into a moderate-scale, PI-led mission for NASA's Solar Terrestrial Probes (STP) line. This community-consensus prioritization led to the STP-5 IMAP mission Announcement of Opportunity (AO) in 2017. On 1 June 2018, NASA announced its selection; this paper describes the selected IMAP science and measurements for the broader Heliophysics community and provides the initial publication and citable reference for this mission.

IMAP investigates two critical and interrelated frontiers of research in Heliophysics, the acceleration of energetic particles (EPs) in interplanetary space and the interaction of the solar wind with the local interstellar medium. We organize the IMAP mission science around the four science objectives specified in the IMAP AO (from the outside in):

(1) improve understanding of the composition and properties of the local interstellar medium (LISM),

(2) advance understanding of the temporal and spatial evolution of the boundary region in which the solar wind and the interstellar medium interact,

(3) identify and advance the understanding of processes related to the interactions of the magnetic field of the Sun and the LISM, and

(4) identify and advance understanding of particle injection and acceleration processes near the Sun, in the heliosphere and heliosheath.

These IMAP science objectives address numerous fundamental and outstanding problems in Heliophysics research, including the

- origin of particle distributions with suprathermal tails and the relative role of discrete episodic events and continuous processes in the production of these tails;

- role of shocks and turbulence in energizing various particle populations; 
Table 1 IMAP Instrument Suite

\begin{tabular}{ll}
\hline IMAP-Lo & $10-1000 \mathrm{eV}$ ISN/ENA fluxes \& composition \\
IMAP-Hi & $0.4-15.6 \mathrm{keV}$ ENA fluxes \& composition \\
IMAP-Ultra & $3-300 \mathrm{keV}$ ENA fluxes \& composition \\
MAG & $2 \mathrm{~Hz}(128 \mathrm{~Hz}$ intervals $) \mathrm{w} / 10 \mathrm{pT}$ resolution \\
SWE & $1-5000 \mathrm{eV}$ electron distribution at $15 \mathrm{~s}$ resolution \\
SWAPI & $0.1-20 \mathrm{keV} / \mathrm{q}$ solar wind ions \& PUIs at $15 \mathrm{~s}$ resolution \\
CoDICE-Lo & Solar Wind/PUI/ST ions $3-60 \mathrm{amu},<0.5-80 \mathrm{keV} / \mathrm{q}$ \\
CoDICE-Hi & $\mathrm{ST} / \mathrm{EP}$ ions 1 to $>60 \mathrm{amu}, 0.03-5 \mathrm{MeV} / \mathrm{nuc} \& 20-600 \mathrm{keV}$ electrons \\
HIT & EP dE/dX vs. E H-Fe ions $2-40 \mathrm{MeV} / \mathrm{nuc} \&$ electrons \\
IDEX & Dust composition $1-500 \mathrm{amu}, \mathrm{m} / \Delta \mathrm{m}>200$ \\
GLOWS & $\mathrm{H}(\mathrm{Ly}-\alpha) \& \mathrm{He}(584 \AA$ glow-global solar wind, ionization, radiation pressure \\
\hline
\end{tabular}

- importance of interstellar pickup ions (PUIs) in the heliosphere and its interstellar interaction;

- source of the enigmatic "Ribbon" of enhanced energetic neutral atoms (ENAs) discovered by the Interstellar Boundary Explorer (IBEX) and its relationship to the higher energy "Belt" seen by the Ion and Neutral Camera (INCA) on Cassini;

- role of the local interstellar magnetic field in shaping the heliosphere;

- structure of the heliotail;

- sources of the lowest-energy ENAs;

- resolution of whether there is a bow shock or wave ahead of the heliosphere;

- composition and properties of the LISM;

- understanding of the highly moderated termination shock.

IMAP creates a complete and synergistic set of observations to address the intimately coupled objectives of particle acceleration and heliospheric interactions based on a complete and synergistic set of observations. These observations simultaneously dissect the particle injection and acceleration processes at 1 AU while remotely probing the global heliospheric interaction and its response to particle populations generated by these processes. In situ at $1 \mathrm{AU}$, this requires detailed observations of the solar wind electrons and ions; EPs, suprathermal ions (STs), and PUIs; and magnetic field. For the outer heliosphere interaction, it requires advanced global observations of the remote plasma and energetic ions over a broad energy range via ENA imaging, and precise observation of interstellar neutral (ISN) atoms penetrating the heliosphere. Complementary observations of interstellar dust (ISD) and ultraviolet (UV) glow of ISNs deepen physical understanding in our baseline mission.

IMAP provides an integrated and coordinated suite of 10 instruments to create the in situ and remote imaging observations of the investigation (Table 1). For each, we chose highheritage instrumentation and made judicious enhancements only where needed to achieve measurement requirements. For the critical heliospheric ENA imaging and ISN atom measurements, our IMAP team also led the IBEX-Lo, IBEX-Hi, and Cassini/INCA instruments; these three instruments effectively define the current state of the art for the required observations of neutrals from $10 \mathrm{eV}$ to $70 \mathrm{keV}$. Direct cross-calibration of IMAP with IBEX, if it is still returning data in 2024, will enable creation of the IBEX-to-IMAP dataset to continue the baseline of time variations in heliospheric ENAs and extend more than 15 years of global ENA imaging.

The IMAP spacecraft and mission implementation are robust and low-risk. The spacecraft is a simple $4 \mathrm{rpm}$ Sun-pointed spinner like IBEX, but in orbit about the Sun-Earth L1 
point, where the terrestrial-related backgrounds are far lower for the ENA measurements and the spacecraft is continuously in the solar wind. The spin axis is re-pointed daily toward the incoming solar wind. Although the baseline mission is accomplished in 2 years, the spacecraft has consumables for at least 5 years, allowing for an extended mission and even larger science returns.

The IMAP science team (Table 2) is drawn from 24 institutions from the US, Germany, Poland, Switzerland, and Japan. The IMAP team seeks to engage the rest of the Heliophysics community in the broadest possible ways. These include (1) welcoming the broad community into IMAP science from the very beginning through joint IBEX/IMAP Science Team meetings that are open to the community, (2) providing \$2M, set-aside from our cost cap, for NASA-selected Guest Investigators who participate as full IMAP team members, as they did on IBEX, (3) providing significant volume, mass, power, and telemetry to accommodate a TDO selected by NASA for flight on our spacecraft, (4) an inclusive Student Collaboration, and (5) an innovative Heliophysics Future Leaders program that engages a set of graduate students and early career scientists and mentors them through the IMAP mission development and science as a way to promote a diverse set of leaders in Heliophysics for the future. We also include an IMAP Active Link for Real-Time (I-ALiRT) real-time data capability, based on the Advanced Composition Explorer (ACE) Real-Time Space Weather that provides critical space weather observations for the community.

\section{Scientific Background}

The Sun's heliosphere, the domain of all human existence, is an astrophysical case history of the development of a habitable star system and an archetype for distant astrospheres that shelter uncounted worlds. Interactions of the heliosphere with the interstellar medium lead to the compression of surrounding magnetic fields and plasmas. The compressed magnetic fields, in turn, lead to the deflection of the majority of galactic cosmic rays (GCRs) around the heliosphere (Zank and Frisch 1999; Scherer et al. 2002; Schwadron et al. 2011). Further study of our global heliosphere, and its myriad interactions with the interstellar medium, is critical for deepening our understanding of the radiation and space environment throughout our inner solar system and understanding our solar system's home within the galaxy.

Similarly, particle acceleration in the heliosphere is a fundamental problem with implications for the future and history of life in the solar system and beyond. Accelerated EPs deposit energy in Earth's, planetary, and exoplanetary atmospheres, and materials from tissue to regolith. It is theorized that chemical changes induced by particle radiation are important to forming the building blocks of life (Todd 1994; Shaviv and Veizer 2003; Rhode and Muller 2005). Yet, for astronauts on future long flights in deep space, high-energy radiation can induce cancer and, at high intensity, lead to radiation sickness (Schwadron et al. 2014a). While particle acceleration occurs throughout the cosmos, the details of the acceleration process remain difficult to discern because it is challenging to directly observe. The heliosphere presents a uniquely accessible environment to study the problem. Highenergy particle acceleration occurs not only at the heliosphere's termination shock, but also at the plethora of shocks and disturbances that travel past Earth with the solar wind.

A remarkable discovery from IBEX (McComas et al. 2009a, 2009b; Schwadron et al. 2009; Fuselier et al. 2009a; Funsten et al. 2009b) and INCA on Cassini (Krimigis et al. 2004,2009 ) is that at the heart of our heliosphere's interstellar interaction lies a population of suprathermal particles that exert enormous pressures, channeling much of the energy from the solar wind into particle acceleration (McComas et al. 2009b; Schwadron et al. 2009; 


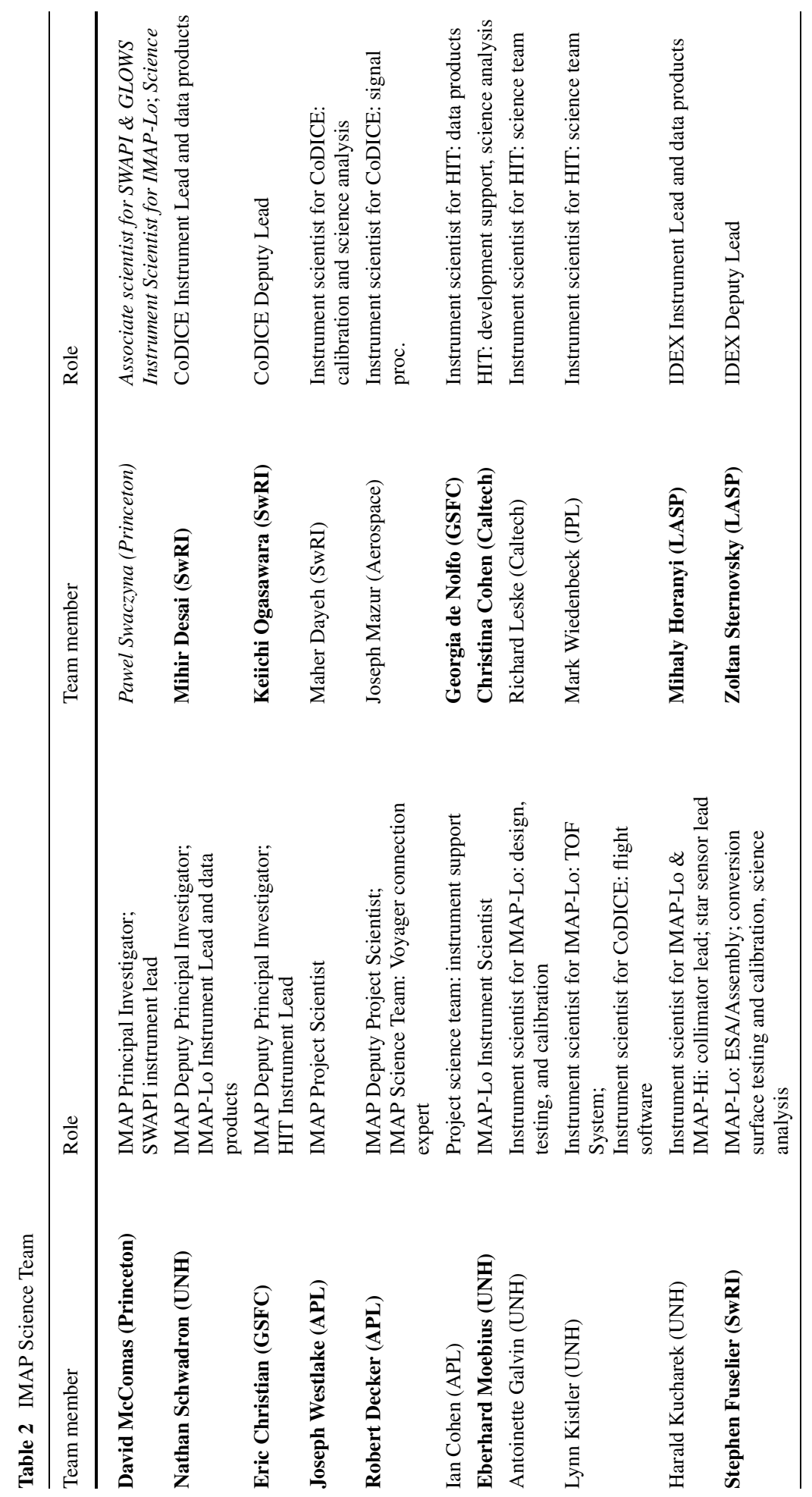




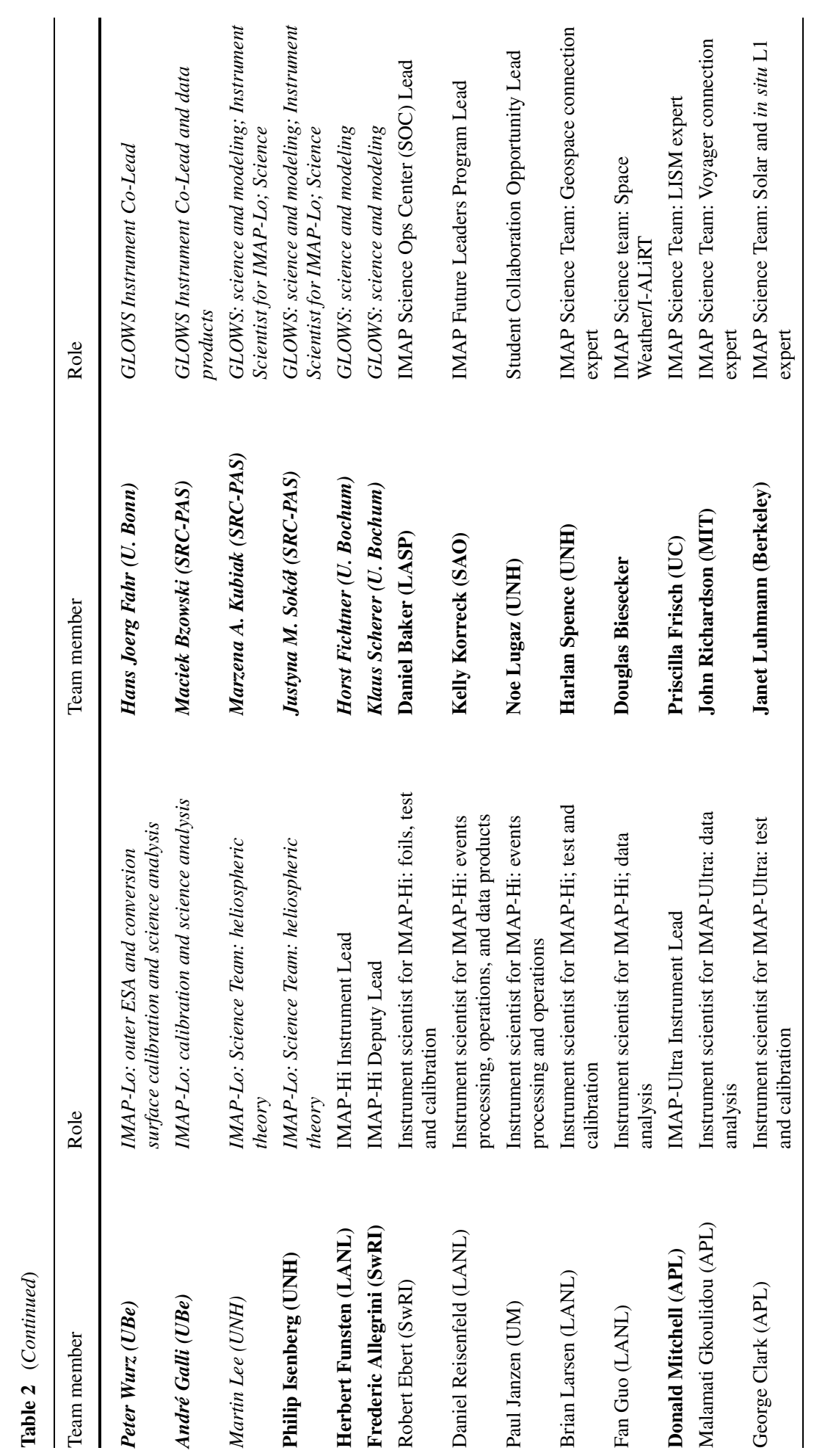




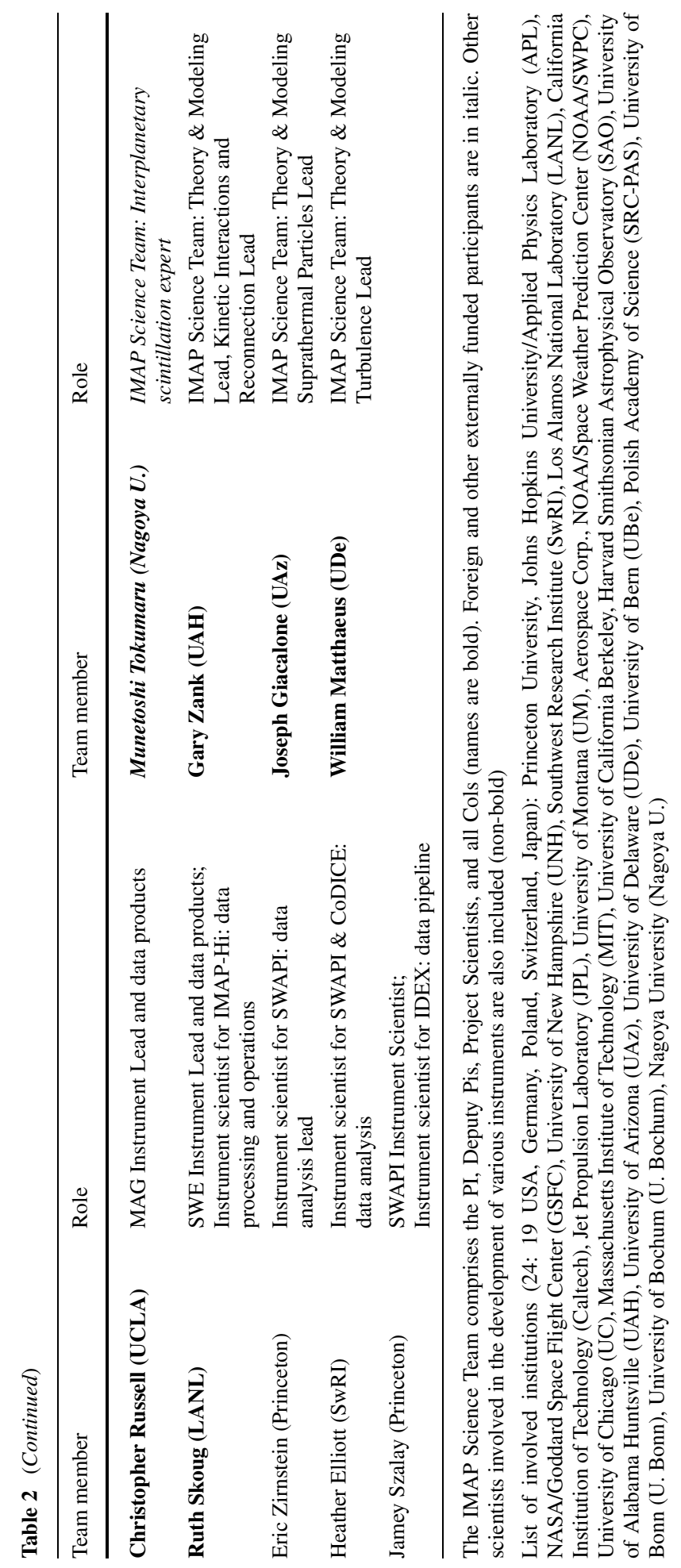




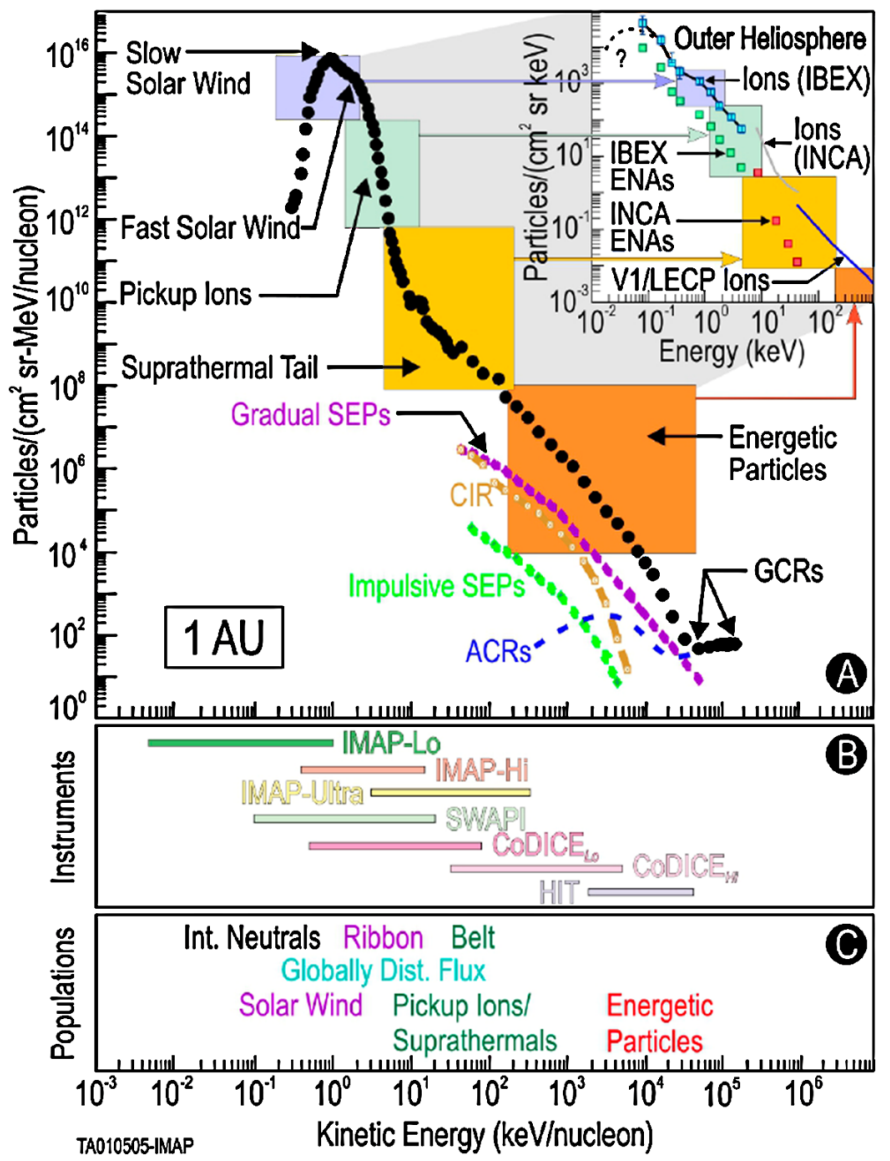

Fig. 1 Measured energy distributions of ENAs, STs, PUIs, and EPs disclose the physical processes that control the acceleration of suprathermal particles at $1 \mathrm{AU}$ and within the heliosheath. Shown here (top panel) are oxygen fluences measured at $1 \mathrm{AU}$ by several instruments onboard ACE during a 3-year period, with representative particle spectra obtained for gradual and impulsive SEPs, corotating interaction regions (CIRs), anomalous cosmic rays (ACRs), and GCRs (adapted from Mewaldt et al. 2001), and (top panel inset) ion fluxes in the Voyager 1 direction using in situ observations from Voyager and remote ENA observations from Cassini and IBEX (Fuselier et al. 2012). (Middle panel) On IMAP, SWAPI, CoDICE, and HIT provide comprehensive composition, energy, and angular distributions for all major solar wind species (core and halo), interstellar and inner source PUIs, suprathermal, energetic, and accelerated ions from SEPs, CME-driven and CIR-associated interplanetary shocks, as well as ACRs. SWE, CoDICE and HIT also provide energy and angular distributions of the solar wind ion and electron core, halo, strahl, as well as energetic and relativistic electrons up to $1 \mathrm{MeV}$

Krimigis et al. 2009). The suprathermal ion population (Fig. 1) extends above 1.5 times the solar wind (or plasma) energy, distinct from the thermal population at lower energies containing the majority of particles. Suprathermal ions, due to their large speed, are preferentially injected into rapid particle acceleration (e.g., Giacalone et al. 1993) and provide the seed populations for EPs.

The IBEX and INCA discovery of large suprathermal populations linked to global interstellar interactions pointed toward what is likely the key to understanding the origins of particle acceleration throughout the cosmos. Similar particle acceleration processes take place 


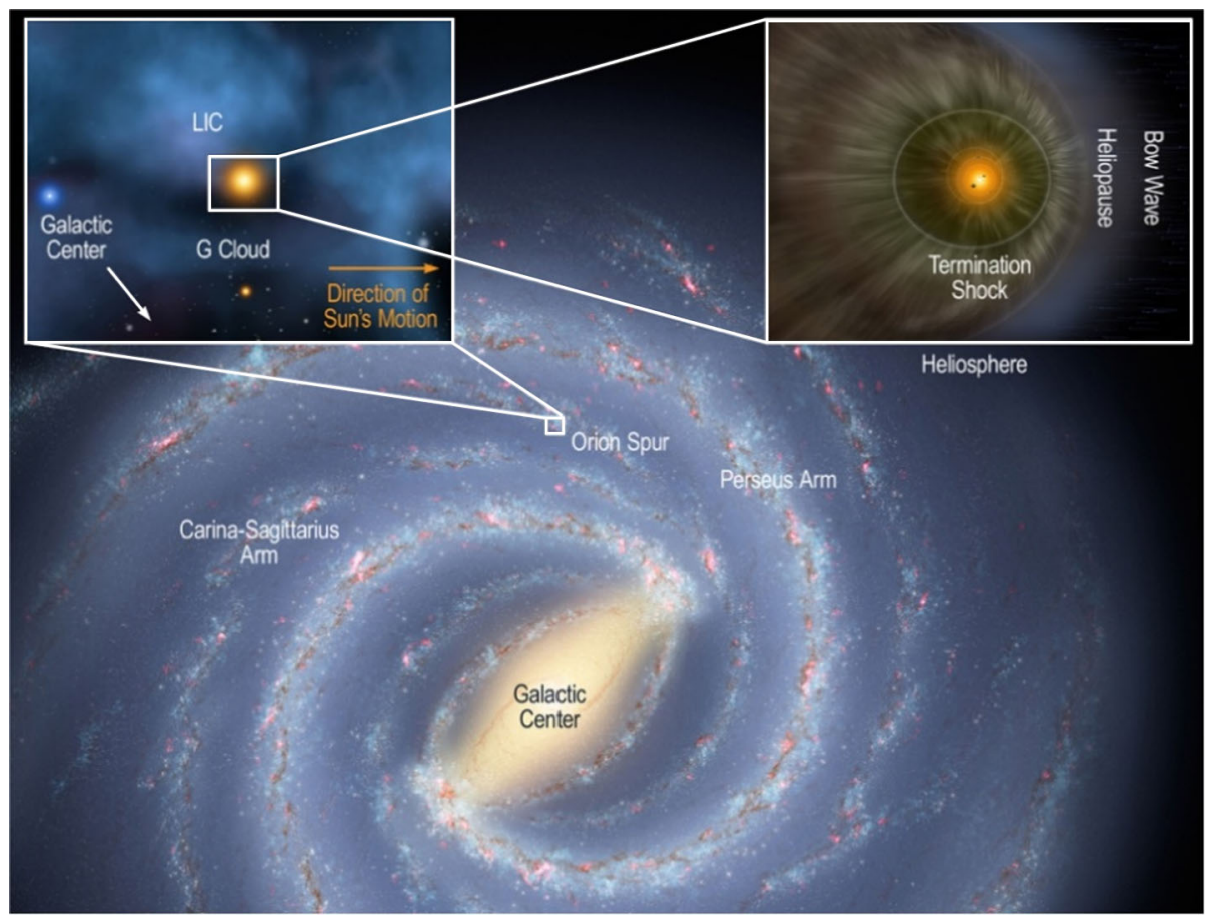

Fig. 2 The galactic environment of the heliosphere. IBEX suggests that the heliosphere is inside the local interstellar cloud (LIC) (McComas et al. 2012, 2015a; Möbius et al. 2012; Bzowski et al. 2012, 2015; Schwadron et al. 2015)

across our solar system, from the shocks and compressional waves ahead of coronal mass ejections (CMEs) that accelerate solar EPs (SEPs) to the populations of accelerated particles near Earth, and in other planetary magnetospheres. Further, these acceleration processes likely extend all the way to a wide variety of astrophysical objects including supernova shocks, the shocks surrounding black holes, and the space environments of exoplanetary worlds. The unified observations and analyses provided by the IMAP mission are crucial to understanding the inextricable link between the origins of particle acceleration and the global interactions of our heliosphere with the LISM.

The solar wind - the supersonic outflow of plasma from the Sun-inflates a bubble in the dilute interstellar medium, the heliosphere. As the Sun travels on its quarter billion-year journey around the galactic center, the size of this bubble has changed in response to the varying interstellar environments (e.g., Frisch 2006) (Fig. 2). The heliosphere, this solarwind-dominated cavity (Online Resource 1), has been an object of study since its existence was first predicted in the 1950s (Parker 1958, 1961).

In October 2008, the tiny $\left(<0.5 \mathrm{~m}^{3}\right.$ and $\left.\sim 100 \mathrm{~kg}\right)$ IBEX spacecraft was launched from Kwajalein Atoll on a Pegasus rocket and began gathering ENA data for the first-ever global maps of the outer heliosphere (McComas et al. 2009a). What the IBEX team found was a feature fundamentally different from anything their prelaunch models of the "ENA sky" or any of the other models of the solar wind/LISM interaction had led anyone to expect: a "Ribbon" of intense ENA emissions nearly encircling the sky and apparently aligned perpendicular to the external interstellar magnetic field (Fig. 3; McComas et al. 2009b; 
Fig. 3 IBEX discovered a "Ribbon" of intense ENA emissions roughly perpendicular to the interstellar magnetic field draped around the heliosphere (McComas et al. 2009b;

Schwadron et al. 2009), which was not anticipated by any prior model or theory

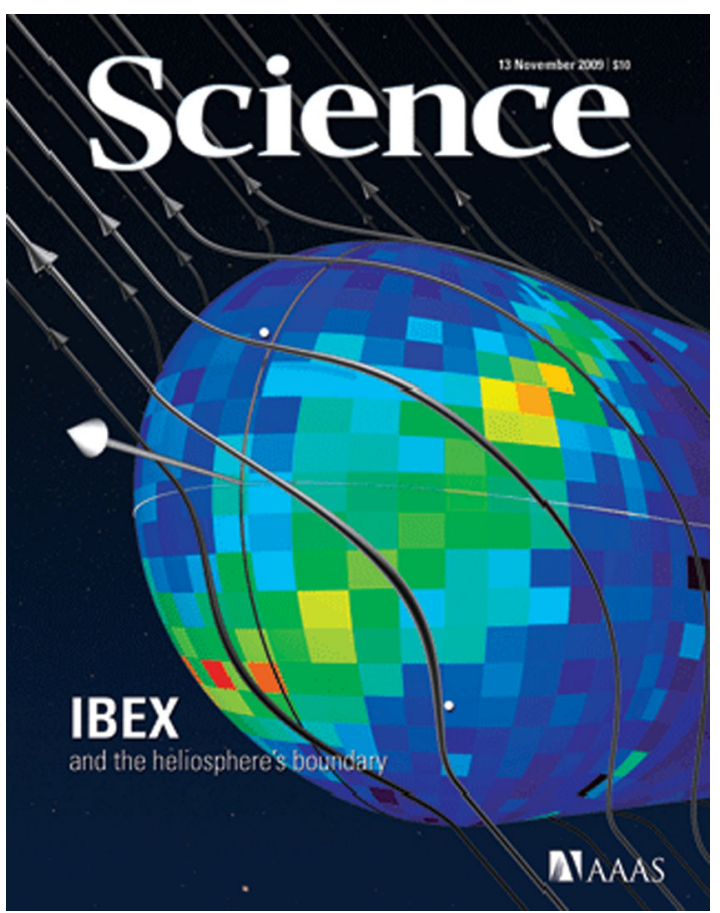

Schwadron et al. 2009). The Cassini spacecraft in orbit about Saturn made similar measurements at higher energies using the INCA instrument and reported a broader "Belt" of enhanced ENA emissions with a spatial distribution similar to, but not the same as, that of the IBEX "Ribbon" (Krimigis et al. 2009).

Our limited understanding of interstellar interactions so far has been developed from: (1) the clever use of limited observations of ENAs from IBEX and INCA (McComas et al. 2009b; Krimigis et al. 2009); (2) the direct in situ data from Voyager 1 and 2 (Zank 2015); (3) observations of ISNs on IBEX (Möbius et al. 2009) and previously on Ulysses (Witte 2004); (4) observations of PUIs on AMPTE, ACE, Ulysses, STEREO, and New Horizons (NH) (Möbius et al. 1985, 1988, 2015b; Gloeckler et al. 1993, 2004; McComas et al. 2017b); (5) ACR observations on ACE, Ulysses, Voyager 1 and 2, and other spacecraft, and (6) observations of solar UV backscattered off the interstellar neutral gas inside the heliosphere (Bertaux and Blamont 1971; Thomas and Krassa 1971; Weller and Meier 1974; Chassefière et al. 1986; Lallement et al. 2005). These observations have also been used in combination with sophisticated 3D global heliosphere models (e.g., Pogorelov et al. 2017b; Zank 1999a). The various observations and models have solved some of the basic questions about the outer heliosphere while simultaneously exposing even more critical knowledge gaps (see Table 3).

IMAP not only sheds new light on PUIs, particle injection, and acceleration, but also provides the $1 \mathrm{AU}$ baseline for the particle input that drives the dynamics of the termination shock, heliosheath, and nearby interstellar medium where ENAs are produced. IMAP is critical for the Heliophysics System Observatory (HSO) by providing comprehensive observational inputs to the geospace environment from orbit around the Sun-Earth L1 point (Fig. 4). 
Table 3 Knowledge gaps and opportunities on the road to interstellar exploration

The IBEX Ribbon (1) remains an enigma. Widely varying sources have been proposed (1-13).

The Ribbon $(1,14,15)$ and Belt $(16,17)$ require conflicting properties for heliosphere structure.

IBEX provided first direct ISN flow measurements of $\mathrm{H}, \mathrm{O}$, $\mathrm{Ne}$ (18), D (19), and Ne/O (20, 21). Precise flow parameters $(\mathrm{H}, \mathrm{O}, \mathrm{Ne})$ and $\mathrm{Ne} / \mathrm{O}$ and $\mathrm{D} / \mathrm{H}$ abundances are unknown.

First radiation pressure from direct ISN H (22) from IBEX conflicts with that from Ly- $\alpha$ measurements.

IBEX observations show a substantially higher LIC temperature than obtained previously (23-25).

IBEX discovered the secondary neutral He component $(26,27)$, but its precise properties remain unknown.

New LISM flow parameters, LISM magnetic field, and PUI modulation suggest no heliospheric bow shock (28-31).

IBEX-derived plasma pressures reveal an asymmetric heliosheath from LISM magnetic pressure $(32,33)$.

The heliosphere is believed to be an "intermediate" case for the LISM flow (34-37). The heliotail may split into two jets $(38,39)$ or is dominated by a strong LISM magnetic field $(40,41)$.

Source of low-energy ENAs $(\sim 0.05-0.5 \mathrm{keV})$ is unknown; competing theories for its origin (42-44).

Co-temporal observations from IMAP and Voyager, IMAP and IBEX fundamentally enable understanding, and provide ground truth for observations, highlighting IMAP's urgency (45).
1. McComas et al. (2009b)

2. Heerikhuisen et al. (2010)

3. Grzedzielski et al. (2010)

4. Chalov et al. (2010)

5. Gamayunov et al. $(2010,2017)$

6. Fahr et al. (2011)

7. Siewert et al. $(2012,2013)$

8. Schwadron and McComas (2013)

9. Kucharek et al. (2013)

10. McComas et al. (2014)

11. Isenberg (2014)

12. Fichtner et al. (2014)

13. Giacalone and Jokipii (2015)

14. Schwadron et al. (2009)

15. Zirnstein et al. (2016b)

16. Krimigis et al. (2009)

17. Dialynas et al. (2017a)

18. Möbius et al. (2009)

19. Rodríguez Moreno et al. (2013, 2014)

20. Bochsler et al. (2012)

21. Park et al. (2014)

22. Schwadron et al. (2013)

23. McComas et al. (2015a, 2015b)

24. Schwadron et al. (2015)

25. Bzowski et al. (2015)

26. Kubiak et al. (2014, 2016)

27. Bzowski et al. (2017)

28. McComas et al. (2012)

29. Pogorelov et al. (2017b)

30. Zank et al. (2013)

31. Heerikhuisen et al. (2014)

32. McComas et al. (2013)

33. Schwadron et al. (2014b)

34. McComas et al. (2013)

35. Pogorelov et al. (2015)

36. Zirnstein et al. (2016a)

37. Zirnstein et al. (2017)

38. Opher et al. (2015)

39. Drake et al. (2015)

40. Krimigis et al. (2009)

41. Dialynas et al. (2017a, 2017b)

42. Desai et al. (2014)

43. Zirnstein et al. (2014, 2018b)

44. Gloeckler and Fisk (2015)

45. Schwadron et al. (2016b) 
Table 3 (Continued)

The origin of suprathermal tails commonly observed in the solar wind (46) remains unknown: suprathermal acceleration has been proposed from a broad array of competing mechanisms (47-50).
46. Fisk and Gloeckler $(2006,2014)$

47. Schwadron et al. (1996, 2010)

48. Livadiotis and McComas $(2009,2013)$

49. Desai et al. (2006)

50. Dayeh et al. $(2009,2017)$

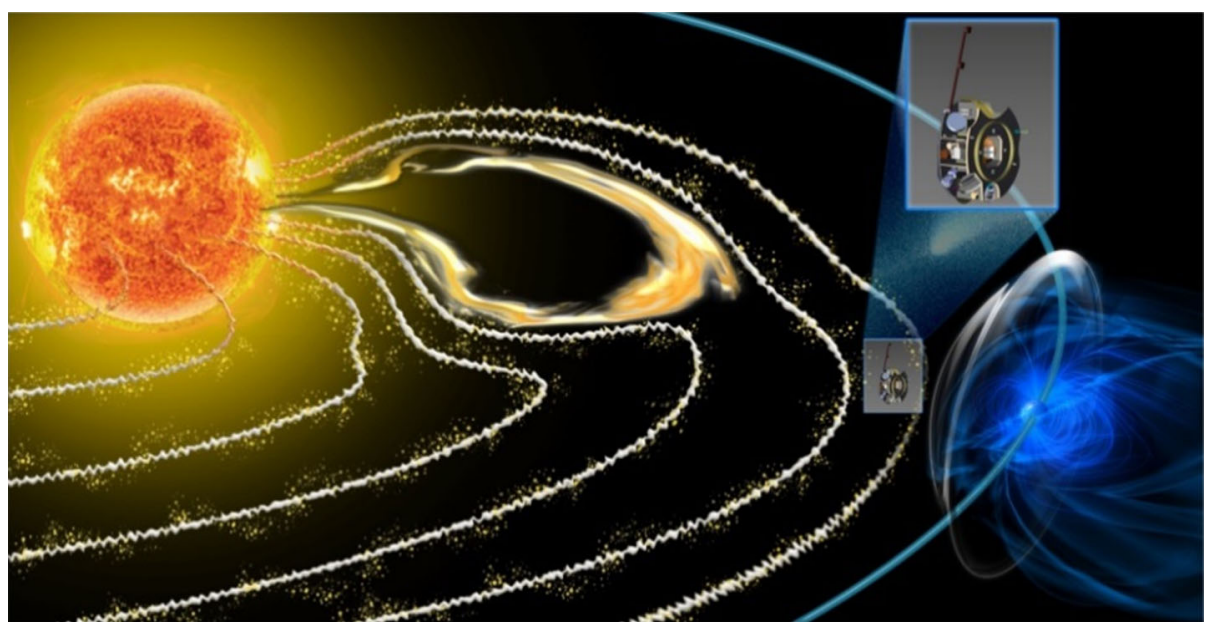

Fig. 4 In orbit around the Sun-Earth L1 point, IMAP is ideally situated and provides comprehensive in situ observations for particle acceleration and L1 science, while providing real-time solar wind observations like that of ACE, but at higher cadences and additional observations needed to enhance real-time predictions

\section{Science Objectives, Goals, and Observations}

IMAP directly addresses and provides closure on four critical science objectives, ordered here from outside the heliosphere inward (without priority):

(O1) Improve understanding of the composition and properties of the LISM.

(O2) Advance understanding of the temporal and spatial evolution of the boundary region in which the solar wind and the interstellar medium interact.

(O3) Identify and advance understanding of processes related to the interactions of the magnetic field of the Sun and the LISM.

(O4) Identify and advance understanding of particle injection and acceleration processes near the Sun, in the heliosphere and heliosheath.

The complete and coordinated observations from the IMAP suite enable fundamental insights through three levels of study (Table 4). First, IMAP allows us to discover global properties of the interstellar interaction as a coupled system, including the origins of particle acceleration directly from IMAP data products. Second, IMAP helps us explore global fundamental properties of the interstellar interactions and origins of particle acceleration using IMAP data products, simple physics-based calculations, theory, and limited 2D and 3D modeling. Finally, IMAP lets us understand in depth the global properties of the interstellar interactions and the origin of particle acceleration through interactive analyses using IMAP data products to define and refine advanced models and simulations. 


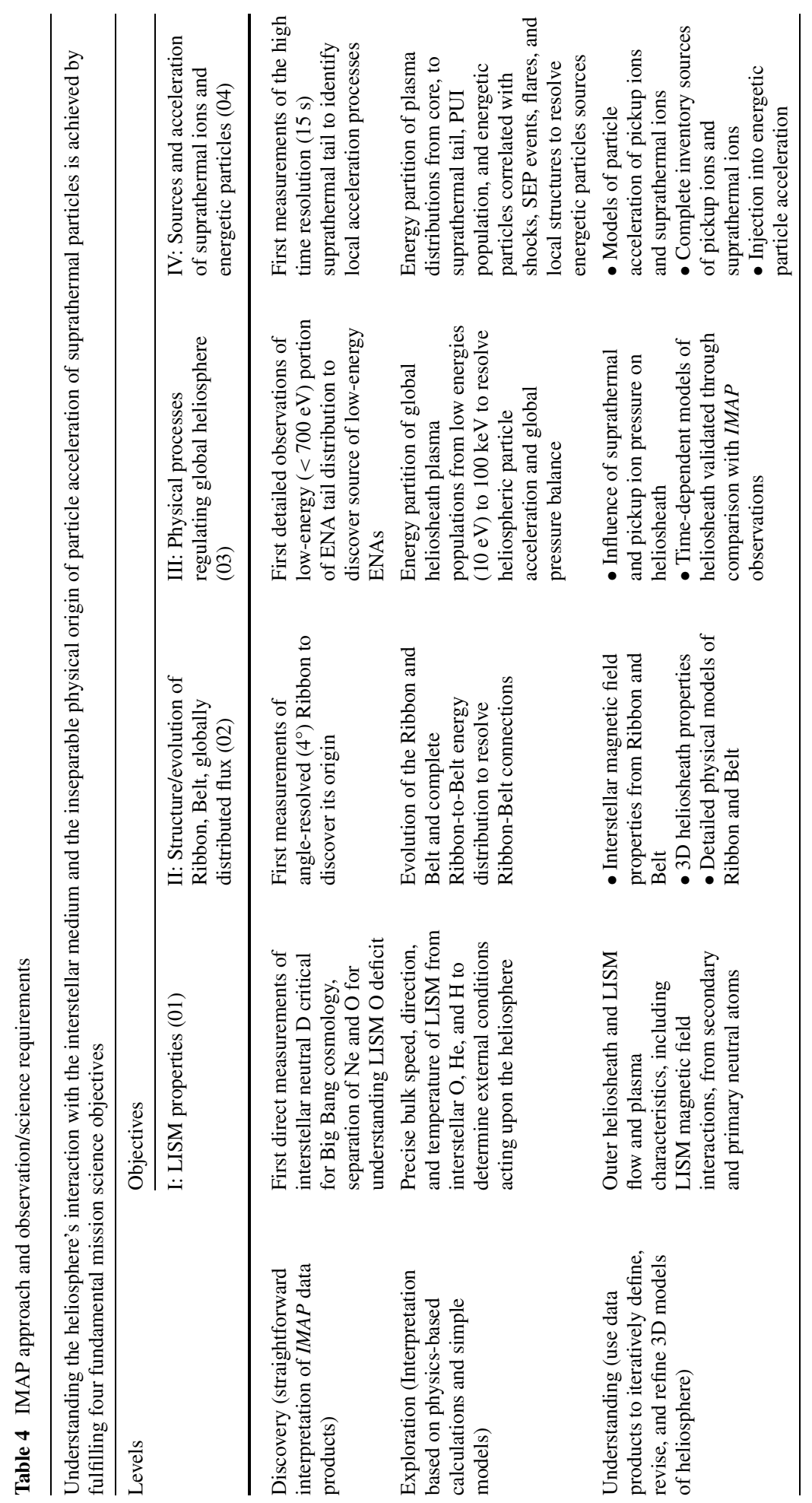




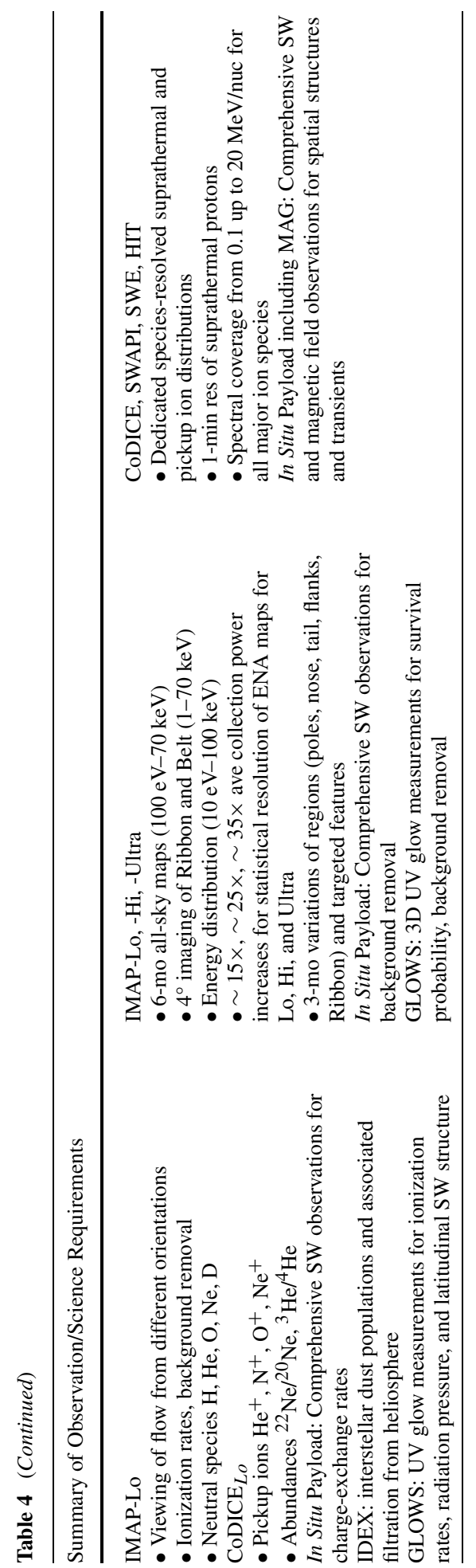


Our IMAP investigation also incorporates robust theory and modeling (T\&M) to gain detailed insight into the global properties of the heliosphere, the Ribbon and Belt, and particle acceleration. The IMAP T\&M team includes Co-Is specifically tasked to ensure deep investigation of (1) the origins of particle acceleration in suprathermal populations, (2) the roles of turbulence in microphysical processes, and (3) kinetic interactions across scales in shocks, interaction and reconnection regions at $1 \mathrm{AU}$, out through the solar wind and into the heliosheath. These T\&M activities connect IMAP's in situ and remote observations, enabling tests of wide-ranging theories through the heliosphere, and closure for open questions at the heart of IMAP. IMAP measurements and T\&M span poorly understood suprathermal particle populations, complementing other HSO missions without expending significant resources to duplicate HSO measurements, as described below.

The four IMAP science objectives lead to five overarching observational drivers:

(1) High-sensitivity global heliospheric imaging;

(2) ENA energy spectra covering core solar wind through EPs;

(3) Neutral $\mathrm{H}, \mathrm{He}, \mathrm{O}, \mathrm{Ne}$, and $\mathrm{D}$ fluxes and flow direction as a function of time of year;

(4) High-time-resolution ( $<1 \mathrm{~min}$ ) PUI, STs, and energetic ion measurements;

(5) Complete solar wind electron, proton, heavy ion, and magnetic field measurements to resolve solar wind structures, CMEs, shocks, and kinetic substructures.

Measurements of ISD, UV glow maps reflecting combined primary and secondary populations of $\mathrm{H}$ and $\mathrm{He}$, and the latitudinal structure of solar wind provide supporting context. These complementary and synergetic observations are required to address fully the four mission science objectives. The summary in Table 4 highlights critical observations required for complete and synergistic IMAP observations, as well as the mapping from the IMAP science Objectives (O1-O4). The following four subsections describe our IMAP mission's approach to achieving each of the four science Objectives in more detail.

\subsection{LISM Properties and Composition (01)}

IMAP observations will allow us to precisely derive the interstellar flow parameters (i.e., interstellar He flow speed and temperature better than $\sim 2 \%$ accuracy, as well as the flow direction) to determine the external boundary condition of the global heliosphere. IMAP provides precise measurements of ISN He, $\mathrm{O}$, and $\mathrm{H}$ to specify the ram pressure of interstellar flow, its Mach number, to meaningfully differentiate the flow parameters of $\mathrm{O}, \mathrm{He}, \mathrm{Ne}$, and $\mathrm{H}$, and to define accurately the critical $\mathbf{B}_{\text {IS }}-\mathbf{V}_{\text {ISN }}$ plane, which organizes the heliosphere structure and topology. Precise measurements of LISM flow properties from IMAP make it possible to provide:

(1) the first determination of outer heliosheath properties that influence our understanding of all secondary neutral populations,

(2) the fundamental physical mechanisms by which the interstellar medium interacts with the solar wind outflow including interstellar flow around the heliopause (Schwadron et al. 2016a),

(3) the charge-exchange coupling with neutral populations within the outer heliosheath (Bzowski et al. 2017), and

(4) the physical processes controlling interactions with the interstellar magnetic field (Pogorelov et al. 2017b; Zirnstein et al. 2016b; Schwadron et al. 2016a).

Ultimately, IMAP's precise measurements are critical for establishing how the interstellar flow interacts with and influences global heliospheric structure, which feeds into objectives $\mathrm{O} 1$ and $\mathrm{O} 2$. 
Current uncertainties prevent accurate determination of the interstellar boundaries. For instance, $\sim 10 \%$ uncertainty in speed translates to $\sim 20 \%$ in interstellar ram pressure. Thus, it is not certain if there is a bow wave or shock (McComas et al. 2012; Bzowski et al. 2015; Kubiak et al. 2016; Zank et al. 2013; Möbius et al. 2015a) ahead of the heliosphere and there are even wide error bars on the orientation of the heliospheric symmetry plane (e.g., Schwadron et al. 2016a). When viewing the interstellar flow from a fixed vantage point (e.g., IBEX-Lo field-of-view (FOV) body fixed perpendicular to spin axis), there is a fixed peak longitude of the observed distribution, which produces an analytic relationship between interstellar flow speed and interstellar ecliptic longitude (referred to as a parameter tube). On IMAP-Lo, the pivot platform allows for viewing from widely separated vantage points (across $>180^{\circ}$ in ecliptic longitude) and therefore produces an array of intersecting parameter tubes, which determine precisely the interstellar speed and flow longitude. The width of the ISN spin-phase distribution is used to determine the interstellar temperature while the peak of the spin-phase distribution also determines the interstellar ecliptic inflow latitude.

Improvements in these observations are essential because, prior to IMAP, direct measurement of ISN atoms by IBEX (McComas et al. 2012; Möbius et al. 2009, 2012; Bzowski et al. 2012) and from Ulysses (Witte 2004) along with direction determinations by PUIs (Drews et al. 2012) presented flow speed differences up to $\sim 10 \%$ and directional differences up to several degrees. While some of these were resolved by invoking a substantially higher interstellar temperature (McComas et al. 2015a; Schwadron et al. 2015; Bzowski et al. 2014, 2015; Möbius et al. 2015a; Wood et al. 2015), systematic effects were uncovered that impact the observations in different ways, including the role of latitudinal structure of the ionization field in the solar wind on the PUI cone location (Sokól et al. 2016) and the discovery of a secondary neutral population (Kubiak et al. 2014, 2016; Park et al. 2016).

IMAP will derive the $\mathrm{O}$ and $\mathrm{H}$ flow speed to better than 5\% accuracy, a significant improvement over the $\sim 20 \%$ accuracy provided by IBEX-Lo (e.g., Schwadron et al. 2016a). The accurate determination of $\mathrm{H}, \mathrm{He}$, and $\mathrm{O}$ flow properties is fundamental for IMAP to resolve the effects of filtration (Schwadron et al. 2016a) and whether the interstellar medium is isothermal or instead strongly influenced by turbulence (e.g., Minter and Spangler 1996; Zank et al. 2017). This advance is essential for understanding the kinetic properties of the local interstellar flow that controls how the interstellar medium physically interacts with the solar wind at the heliopause. IMAP enables a more detailed understanding of the interstellar conditions, which is equally critical in determining how the Ribbon and Belt form in the LISM (e.g., McComas et al. 2014; Heerikhuisen et al. 2010) and is associated with objective O3. Precise determination of the ISN parameters and the secondary components provides tight constraints on the ionization state of the LISM (e.g., Frisch et al. 2011).

The LISM is the only locally accessible sample of extrasolar material and thus provides the state of galactic matter today in juxtaposition to solar material from 4.5 billion years ago. It therefore provides us with critical insight into Big Bang nucleosynthesis and the galactic evolution in our neighborhood, which is representative of the galactic habitable zone. Measurements of ${ }^{3} \mathrm{He}^{+} /{ }^{4} \mathrm{He}^{+}$from PUIs and D/H from ISN atoms are key observations for Big Bang nucleosynthesis, with ${ }^{3} \mathrm{He}^{+} /{ }^{4} \mathrm{He}^{+}$thus far observed to only $\pm 20 \%$ (Gloeckler and Geiss 1998) and there are no in situ $\mathrm{D} / \mathrm{H}$ observations. However, a first determination of D/H in the LISM was accomplished with IBEX (Rodríguez Moreno et al. 2013, 2014). Interstellar $\mathrm{D} / \mathrm{H}$ is a powerful probe of nucleosynthesis and the chemical evolution of the Milky Way galaxy (e.g., Linsky 2003, 2007), yet observations in the interstellar medium are scarce and line-of-sight integrated (Linsky et al. 2006) and ionization levels of interstellar atoms may vary significantly between the heliosphere edge and interior of the surrounding interstellar 
cloud (Slavin and Frisch 2008). The D/H ratio provides input on stellar evolution, and its contribution to the local matter inventory and the $\mathrm{Ne} / \mathrm{O}$ ratio has ramifications on the balance between volatile and refractory elements (e.g., Jenkins 2009), which requires observation of gas phase and dust composition in the LISM.

IMAP will provide the LISM composition measurements needed to move beyond qualitative constraints (Bochsler et al. 2012; Bzowski et al. 2013a; Park et al. 2014; Gloeckler and Geiss 1998; Frisch and Slavin 2002; Linsky 2003, 2007; Slavin and Frisch 2008) and include the first detailed abundance information from direct ISN matter. For $\mathrm{D} / \mathrm{H}$, this will be to better than $20 \%$ accuracy, ISN Ne/O to better than $10 \%$ accuracy, ${ }^{3} \mathrm{He}^{+} /{ }^{4} \mathrm{He}^{+}$PUI abundance to better than $10 \%$ accuracy, and ${ }^{22} \mathrm{Ne}^{+} /{ }^{20} \mathrm{Ne}^{+}$PUI abundance to better than $30 \%$ accuracy. The first accurate interstellar $\mathrm{Ne} / \mathrm{O}$ abundance ratio from IMAP will test the existence of an oxygen deficit in the local galactic medium due to preferential processing of $\mathrm{O}$ on grains (Schwadron et al. 2016a; Slavin and Frisch 2008; Altobelli et al. 2016). IMAP PUI abundance information (i.e., ${ }^{22} \mathrm{Ne}^{+} /{ }^{20} \mathrm{Ne}^{+},{ }^{3} \mathrm{He}^{+} /{ }^{4} \mathrm{He}^{+}$) specifies LISM isotopic properties key to galactic evolution and nucleosynthesis models.

IMAP also provides the first accurate in situ measurements of the flux, size distribution, and composition of ISD particles flowing through our solar system. IMAP will provide detailed composition measurements (mass resolution $\mathrm{m} / \Delta \mathrm{m}>200$ ) of 100 particles per year based on Ulysses-derived fluxes (Landgraf et al. 2003) to fill a significant knowledge gap in our current limited compositional measurements of ISD particles. The Cassini Cosmic Dust Analyzer (CDA) orbiting Saturn found 36 ISD particles all with similar chemical makeup of magnesium rich silicate and oxide composition with iron inclusions (Altobelli et al. 2016). The Stardust mission returned even fewer (only 7) candidate ISD particles with diverse chemical composition, including sulfides in some of the particles that contradict the CDA results (Westphal et al. 2014). The much higher IMAP observation rate provides an unparalleled opportunity to directly sample and thereby discover the chemical makeup of solid matter in our galactic environment with unmatched resolution and move beyond the limitations of these prior measurements.

Finally, IMAP incorporates detailed information of the UV glow from inflowing $\mathrm{H}$ and He. These observations provide complementary ionization and radiation pressure measurements (amplitude variations better than 5\% accuracy) and solar wind measurements (resolving latitude variations better than $15^{\circ}$ accuracy) needed to interpret the directly sampled ISN H, Ne, and O. Observations of the backscatter He glow informs on the solar wind electron temperature inside $1 \mathrm{AU}$ at all heliolatitudes and constrains the electron ionization rate of ISN gas. These measurements provide complementary knowledge of ionization rates, which cull ISN atoms, thereby influencing the observed spatial and velocity distributions of ISN H, O, He, Ne, and D. Direct ionization rate measurements significantly improve our understanding of the ISN flow properties (Sokół et al. 2015b, 2016). Current knowledge of electron ionization rate inside $1 \mathrm{AU}$ is only thought to be accurate to within an order of magnitude (Lallement et al. 2004a), but likely significantly attenuates the flux of the indirect beams of ISN atoms (Ruciński and Fahr 1991). The pivot platform will enable IMAP-Lo to observe also the indirect beam and thereby provide an independent measurement of the total ionization rates at least for $\mathrm{He}$ and $\mathrm{Ne}$.

\subsection{Structure and Evolution of Ribbon, Belt, Globally Distributed Flux (O2)}

IBEX's ENA sensors provided novel measurements that shaped our understanding of the heliosphere, while Cassini/INCA provided further insight with higher-energy ENA measurements. However, both IBEX and INCA face challenges resolving the faint ENA emissions from the outer heliosphere. For example, Cassini observations were limited by Saturn's 

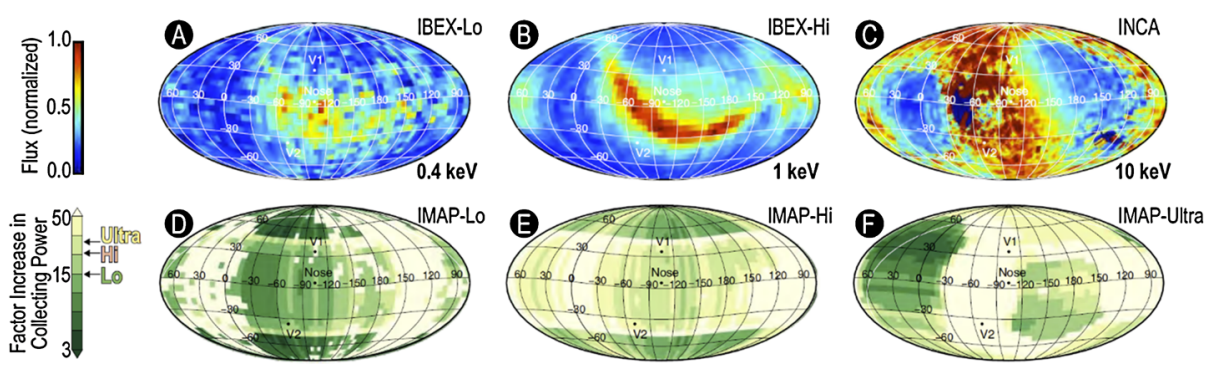

Fig. 5 (A-C) IBEX-Lo, -Hi, and Cassini/INCA produced the first maps of the global ENA distributions. (D-F) IMAP-Lo, -Hi, and -Ultra provide much larger collecting power (CP) than their predecessors that enables all-sky maps and complete energy spectra. CP shown in $(\mathbf{D})$ is based on the IMAP-Lo pivot platform pointing $50 \%$ of the time at $0^{\circ}$ and $50 \%$ at $45^{\circ}$; however, smaller regions can be imaged with even higher CPs

large ENA source region and the small amount of time spent outside of Saturn's magnetosphere. IMAP incorporates major advancements to overcome the challenges faced by IBEX and INCA, including an average improvement in collection power of $\sim 15 \times, \sim 25 \times$, and $\sim 35 \times$ for IMAP-Lo, IMAP-Hi, and IMAP-Ultra, respectively; introduction of a pivot platform to optimize sky viewing and measure the interstellar inflow for most of the year; and the ability to resolve temporal changes down to 3 months (IMAP-Lo and IMAP-Hi) and 1 week (IMAP-Ultra). These leaps in spatial and temporal resolution, and precise determination of interstellar properties, yield unprecedented resolution of interstellar interactions.

IMAP resolves the temporal and spatial evolution of the Ribbon, the Belt, and interstellar boundary regions. IMAP provides high angular resolution $\left(4^{\circ}\right)$ global ENA maps from 1 to $70 \mathrm{keV}$ (for estimated counting statistics) to resolve Ribbon-Belt spatial structure. IMAP also produces global maps extending down to $100 \mathrm{eV}$ to cover the complete Ribbon-Belt ENA energy range, albeit at somewhat lower angular resolution. Finally, IMAP measures the complete ENA energy spectra $(10 \mathrm{eV}-100 \mathrm{keV})$ from larger, but distinct regions (e.g., nose, tail, flanks, poles, Ribbon, Belt) to define the morphology and evolution of heliosheath populations from the plasma core, to STs and EPs. Observations spanning this complete energy range are critical for establishing not only the origin of the Ribbon but also its relationship to the Belt.

IMAP also probes the evolution of the heliosphere on short, 3-month scales, to test for the existence of instabilities near the nose and heliopause (Pogorelov et al. 2017a, 2017b; Zank 1999b; Borovikov and Pogorelov 2014), and possible rapid evolution of the Ribbon and Belt (McComas et al. 2017a). These time-resolved measurements of the Ribbon, Belt, and other structures probe from the Ribbon and PUI populations up through suprathermal and EP energies. IMAP provides much higher collection power than previous observations, with $\sim 15 \times, \sim 25 \times, \sim 35 \times$ that of IBEX-Lo, IBEX-Hi, and INCA, for IMAP-Lo, IMAP-Hi, and IMAP-Ultra, respectively, on average (Fig. 5).

Different Ribbon origin hypotheses predict different key observables, such as the global spatial geometry, global spectral properties, location relative to the Sun's motion through the LISM, and variability of the ENA emission intensity over space and time (McComas et al. 2014). In particular, for hypotheses in which the ENAs originate as solar wind ions, the temporal variability of ENA flux and its association with solar wind variation is emerging as a definitive test (e.g., Zirnstein et al. 2015b). A recent model highlights the need for high-angular-resolution measurements of the Ribbon. That model suggests that the Ribbon forms from the interaction of PUIs beyond the heliopause with preexisting interstellar 


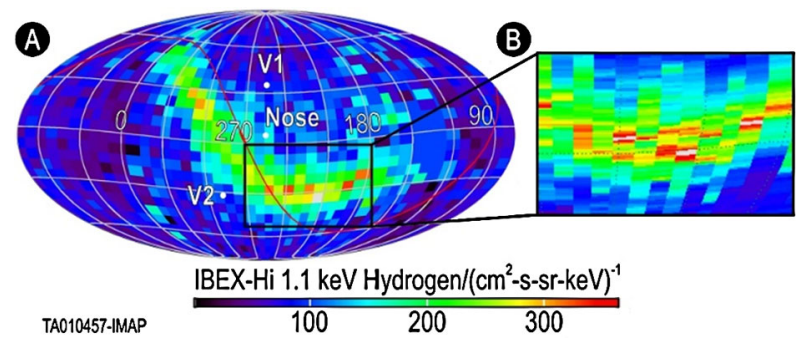

Fig. 6 Mollweide projection showing IBEX "Ribbon" of enhanced ENA emissions from McComas et al. (2009b). Prime motivations for IMAP are the resolution of possible fine structure to discover the origin of the Ribbon, and its connection to the Belt. (A) All-sky ENA map showing the IBEX Ribbon at $1.1 \mathrm{keV}$, including locations of the Nose and Voyager. (B) Detail of a segment showing apparent fine structure

turbulence via magnetic mirroring near $\mathbf{B}_{\text {IS }} \cdot \mathbf{r} \sim 0$ (Giacalone and Jokipii 2015). This mechanism predicts that the Ribbon is double-peaked due to particle-wave interactions; this may be reflected in the possible fine structure seen in early IBEX observations (Fig. 6). The existence of Ribbon fine structure (or a double-peak) is unconfirmed. The improved IMAP angular resolution (down to $4^{\circ}$ for energies $>1 \mathrm{keV}$ ) is sufficient to discover fine structure associated with instabilities in ribbon and Belt formation, and potentially other heliosphere structures.

\subsection{Physical Processes Regulating the Global Heliosphere (O3)}

IMAP resolves how physical processes within the heliosheath control global heliospheric structures as well as the interactions among the solar wind, the local interstellar flow, and local interstellar magnetic field. The approach to this objective involves six synergistic sets of observations as follows.

ISN atom populations and PUIs reflect the external properties of the interstellar medium imposed as boundary conditions on the global heliosphere. With IMAP, we observe the primary ISN populations $(\mathrm{H}, \mathrm{He}, \mathrm{O})$ to determine precise interstellar flow properties. These observations set the outer boundary conditions on the global heliosphere and regulate the heliosphere's interstellar interaction.

Energy partition between the various ENA populations is critical to determining the physical processes that regulate the heliosphere. With IMAP, we resolve the complete ENA energy spectrum by measuring ENA populations from core solar wind through PUI, STs, and EPs with statistical accuracy across the full energy range from $10 \mathrm{eV}$ to $100 \mathrm{keV}$.

Heliospheric structures, and the resulting distributions of ENA emissions, evolve over time on various characteristic timescales (e.g., McComas et al. 2018; Zirnstein et al. 2018a). IMAP resolves the evolution of ENA fluxes down to six-month timescales to track the temporal evolution of the Ribbon, Belt, and heliospheric regions at energies down to $100 \mathrm{eV}$. These observations allow us to understand the temporal variability of global heliospheric structures.

Observations available from IBEX and INCA have not been sufficient to fully resolve the differences between the Ribbon and Belt and understand the connection between them. This lack of understanding further clouds the relationship between the different orientations of these two structures and how one or both reflect the external direction and strength of the interstellar magnetic field. On IMAP we resolve the Ribbon and Belt connection and direction and the strength of the interstellar magnetic field (e.g., Zirnstein et al. 2016b) 
with a combination of high-angular-resolution ENA measurements $\left(4^{\circ}\right)$ spanning $100 \mathrm{eV}$ to $70 \mathrm{keV}$ and ENA energy distributions with lower angular resolution (to increase counting statistics) up to $\sim 100 \mathrm{keV}$. These observations are taken by a suit of three instruments that are properly intercalibrated over overlapping energy ranges.

The charge-exchange process depletes the energetic protons in the heliotail and makes the interpretation of the heliotail structure difficult (e.g., Schwadron et al. 2014b; Schwadron and Bzowski 2018). With the ENA composition measurement from IMAP-Hi, we also observe ENAs of elements heavier than $\mathrm{H}$. Lower charge-exchange rates for energetic He ions allows their penetration into more distant regions of the heliotail and the production of $\mathrm{He}$ ENAs will help expose the global heliotail structure (Swaczyna et al. 2017; Swaczyna and Bzowski 2017).

Secondary neutral atom populations arise out of the interaction of the heliosphere with the LISM. As the interstellar material flows around the heliosphere, it is heated and deflected. With IMAP-Lo, we accurately resolve secondary ISN populations (of $\mathrm{H}, \mathrm{He}$, and $\mathrm{O}$ ) to derive interstellar flow deflection and heating around the heliopause. The counting rates of secondary atoms are typically $\sim 10 \%$ of their primary populations (e.g., Park et al. 2016). IMAP observes these secondary species from multiple vantage points over the year, as needed to understand the 3D spatial structure of the interstellar flow near the nose (e.g., Bzowski et al. 2017) and the distribution function of interstellar neutrals in the LISM (Sokól et al. 2015a).

Finally, on IMAP we relate in situ observations of the origin of suprathermal populations and energetic populations at $1 \mathrm{AU}$ to the ENA energy distributions. This connection lets us determine the physical processes mediating the plasma balance within the heliosheath. We do this by understanding the origin of suprathermal particles from highly variable in situ measurements ( $\sim 1 \mathrm{~min}$ resolution) at $1 \mathrm{AU}$ covering populations from solar wind core protons through suprathermal and energetic protons $(\mathrm{O} 4)$.

\subsection{Sources and Acceleration of Suprathermal Ions and Energetic Particles (O4)}

To discover the origins of accelerated particles near the Sun, in the heliosphere and heliosheath, IMAP provides:

(1) high-time-resolution ( $\sim 1 \mathrm{~min}$ ) observations of solar wind protons, suprathermal proton tails, and accelerated protons $(0.2 \mathrm{keV}-20 \mathrm{MeV}$ protons);

(2) energy coverage $(0.7 \mathrm{keV} / \mathrm{q}-20 \mathrm{MeV} / \mathrm{nuc})$ of heavy ions (He-Fe) at 1 -hour resolution for resolving EP source populations and injection into acceleration with adequate energy resolution $(\Delta \mathrm{E} / \mathrm{E})$ to resolve energy spectral features and slopes within suprathermal tails;

(3) angular distributions ( $15^{\circ}$ resolution, $0.7-70 \mathrm{keV} / \mathrm{q}$ at 1 hour for $\mathrm{He}^{+}$) for PUI ring, shell distributions and scattering, and angle-averaged energy distributions $\left(0.7-70 \mathrm{keV} / \mathrm{q}, \mathrm{O}^{+}\right.$, $\mathrm{N}^{+}, \mathrm{Ne}^{+}$at 12 hours and $\mathrm{He}^{+}$at 10 minutes) for interstellar PUIs throughout the year and through the gravitational focusing cone for independent interstellar He flow direction derivation (Möbius et al. 2015b) from PUIs;

(4) anisotropies (0.05-20 MeV/nuc ions, 30-500 keV electrons) at 1-minute resolution for protons, He, and electrons in directions sunward, anti-sunward, and perpendicular to the magnetic field to identify transport effects in remote acceleration events; and

(5) ion composition (0.05-20 MeV/nuc) ${ }^{3} \mathrm{He} /{ }^{4} \mathrm{He}<10 \%$ level, $\mathrm{C} / \mathrm{O}<50 \%$ level, $\mathrm{Fe} / \mathrm{O}<$ $10 \%$ at 1 -hour resolution for identifying seed populations and distinguishing impulsive and gradual SEPs. 
IMAP also leverages results from HSO missions (e.g., MMS, ISEE, Cluster, ACE, Wind, etc.) and focuses IMAP on science targets that fill gaps in our existing knowledge and capabilities, i.e., simultaneous measurements of the magnetic field and the poorly measured suprathermal ion populations. There are only a few observations of ion acceleration in solar wind shocks at suprathermal energies (e.g., Bamert et al. 2002) and for solar wind turbulence (Bamert et al. 2008; Kallenbach et al. 2006). More recently, energization by turbulence and magnetic reconnection are studied in unprecedented detail by MMS (Burch et al. 2016a, 2016b; Chasapis et al. 2017), while acceleration at the Earth's bow shock at ion kinetic timescales is studied by ISEE, Cluster, MMS (Russell et al. 1982; Gosling et al. 1978; Scholer et al. 2005; Mazelle et al. 2003; Johlander et al. 2016; Meziane et al. 2007; Escoubet et al. 2013; Mauk et al. 2016). In concert, the IMAP observations represent the complete in situ measurements needed to discover the origin of suprathermal tails from particle acceleration. The $\sim 1$-minute timescale extends into the dissipation range of turbulence (e.g., Matthaeus and Velli 2011) and is needed to separate discrete episodic events in contrast to continuously active processes in the production of suprathermal tails (e.g., Fisk and Gloeckler 2014; Schwadron et al. 1996).

On IMAP, identifying the sources and mechanisms of particle acceleration near interplanetary shocks imposes the most stringent requirements because they move rapidly past IMAP. IMAP MAG transmits magnetic field vectors at $2 \mathrm{~Hz}$, capturing the entire inertial range of ambient solar wind turbulence (Bruno and Carbone 2016). For 15 minutes each day MAG transmits in high rate mode at $128 \mathrm{~Hz}$, capturing kinetic range samples over a wide range of solar wind conditions. This unbiased high rate mode eliminates the need for a burst mode that would create biased samples, associating suprathermal transients only with prescribed trigger conditions. ACE and Wind data show that $>45 \mathrm{keV}$ suprathermal ion intensities above $45 \mathrm{keV}$ exhibit dynamic changes at time scales of $\sim 10$ min upstream of interplanetary shocks (Giacalone 2012), whereas simulations show shorter timescales (Giacalone et al. 1993; Giacalone 2004). On IMAP, we identify particle acceleration upstream of shocks from the intensities of $\sim 4-200 \mathrm{keV} / \mathrm{n}$ suprathermal ions at high cadence of $15 \mathrm{~s}$ to 1 minute. To identify the EP sources, we observe high-energy resolution $(\Delta \mathrm{E} / \mathrm{E}<15 \%$ at $10 \mathrm{keV}$ and $<40 \%$ at $200 \mathrm{keV}$ ) and temporal variations in spectral features near shocks. Sub-minute cadences enable detailed studies of potential trapping and reacceleration regions downstream of shocks (Tessein et al. 2013) and secondary islands (Khabarova and Zank 2017). Other acceleration mechanisms, e.g., solar wind velocity fluctuations, compressional turbulence, the so-called pump mechanism, and reconnection islands (Fisk and Gloeckler 2012; Khabarova et al. 2016, 2017), have longer timescales and are easily tracked with cadences of $15 \mathrm{~s}$ to 1 minute.

The suprathermal observations required for IMAP are made in conjunction with solar wind ion measurements to resolve the relationship between accelerated PUIs and suprathermal particles with solar wind structures; fast, slow, and transitional flows; turbulence; transients; and CMEs. The necessary solar wind observations on IMAP include solar wind heavy ion composition measurements of $\mathrm{C} / \mathrm{O}, \mathrm{Ne} / \mathrm{O}$, and $\mathrm{Fe} / \mathrm{O}$ at better than $30 \%$ accuracy and 1 hour resolution (Aellig et al. 1998a), charge state measurements of $\mathrm{C6}^{+} / \mathrm{C}^{+}, \mathrm{O}^{+} / \mathrm{O6}^{+}$, and $\mathrm{Fe}(\mathrm{low}) / \mathrm{Fe}$ (high) at better than $30 \%$ accuracy and 1 hour resolution (Aellig et al. 1998b; Hefti et al. 1998), solar wind ion and electron moments (speed and density determinations of better than $10 \%$ accuracy at $15 \mathrm{~s}$ resolution), and magnetic field observations of resolution $10 \mathrm{pT}$ up to $128 \mathrm{~Hz}$ cadence for resolving shocks and turbulence.

IMAP continuously observes ENA energy spectra covering the energy range of particle injection (100 eV to $100 \mathrm{keV}$ ) into diffusive shock acceleration (e.g., Zank et al. 2007) and stochastic particle acceleration from the outer heliosphere (e.g., Zirnstein et al. 2018b). By examining the variability of these ENA energy spectra as a function of location within 


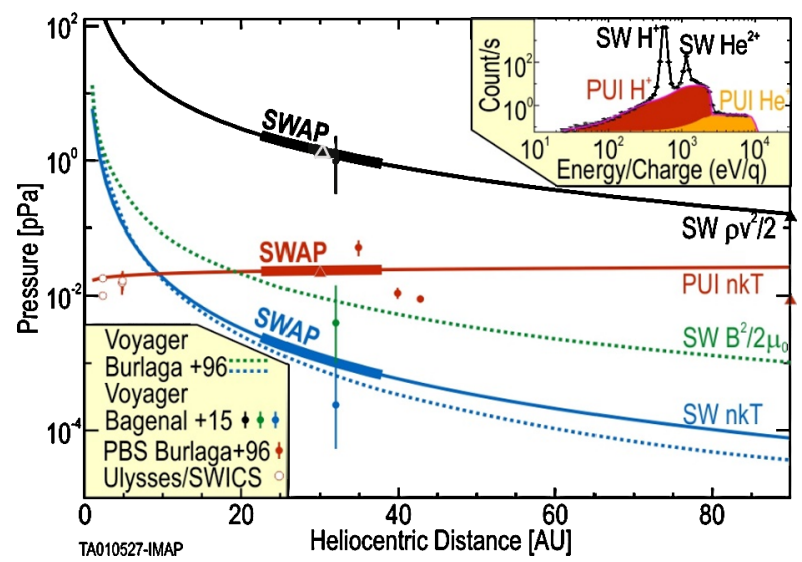

Fig. 7 SWAP energy spectrum (inset) and radial trends from McComas et al. (2017b). Observations (thick lines) from 22 to $38 \mathrm{AU}$ and extrapolated radial trends (thin lines) of the solar wind dynamic pressure (black) and solar wind thermal pressure (blue) as well as the pickup $\mathrm{H}^{+}$pressure (red). Magnetic pressure (green dashed) and another measure of solar wind particle pressure (blue dashed) are from Voyager (Burlaga et al. 1996). Averaged values and ranges at 33 AU come from Bagenal et al. (2015). Additional measurements of the PUI pressure from the Ulysses SWICS instrument out to $\sim 5 \mathrm{AU}$ and inferred values from pressure balance structures (PBSs; Burlaga et al. 1996) are plotted in red

the heliosheath (nose, flanks, tail, poles, latitude structure; e.g., Desai et al. 2016a), IMAP provides global measurements to explore how PUIs and suprathermal ions are injected and accelerated across the termination shock, within the heliosheath, and in the Ribbon and Belt.

The populations observed at 1 AU including PUIs from ISNs, suprathermal ions, and EPs are also present in the heliosheath globally distributed flux (e.g., Schwadron et al. 2014b; Zirnstein et al. 2017) and likely in the Ribbon and Belt (e.g., Schwadron and McComas 2013; Zirnstein et al. 2015a, 2016b), suggesting that the physical understanding of particle acceleration and suprathermal ions at $1 \mathrm{AU}$ should feed directly into our understanding of these processes active in the outer heliosphere and LISM. It must be emphasized, however, that the solar wind at $1 \mathrm{AU}$ is quite different from the regimes of the outer heliosphere. For example, PUIs become the dominant source of internal pressure in the outer heliosphere (McComas et al. 2017b; Zirnstein et al. 2018c) and therefore participate in the heliosheath dynamics to a much larger extent there than at 1 AU (e.g., Zank et al. 1996, 2014; Zank 2016). Thus, a central opportunity with IMAP is to develop the physical understanding of suprathermal particles so that it can be applied across the broad regimes from $1 \mathrm{AU}$ out through the heliosphere.

Lastly, it is critical for IMAP to "connect the dots" between 1 AU in situ observations of particle acceleration and PUIs with the populations observed in the distant solar wind (but still inside the termination shock). This connection is essential because these populations feed into the termination shock, emerging downstream in the inner heliosheath as the shocked, heated, and slowed populations that become PUIs and suprathermal ENAs observed directly by IMAP (e.g., Kumar et al. 2018; Zirnstein et al. 2018c). Closing the observational triad between 1 AU (IMAP in situ data), the outer heliosphere supersonic solar wind - from complementary NH SWAP data (Fig. 7) —and the heated and slowed plasma of the inner heliosheath (from IMAP ENA data) is essential to fully resolving and connecting the origin of particle acceleration with global heliospheric properties and evolution (O2-O4). Global outer heliospheric modeling (e.g., Zirnstein et al. 2017, 2018a; McComas et al. 2018) provide essential tools for closing this observational triad. 
Table 5 IMAP-Lo instrument performance

\begin{tabular}{ll}
\hline Parameter & Performance \\
\hline Instrument Type & Single-Pixel ENA Imager \\
FOV & $9^{\circ} \times 9^{\circ}$, pivoted FOV $60^{\circ}-180^{\circ}$ to spin axis \\
Energy Range & $5-1000 \mathrm{eV}$ \\
Pointing Knowledge & $0.1^{\circ}$ \\
GE $\left(\mathrm{cm}^{2}\right.$ sreV/eV) & $1.9 \times 10^{-4} \mathrm{He} ; 2.2 \times 10^{-4} \mathrm{O} ; 7.8 \times 10^{-5} \mathrm{H}$ at $100 \mathrm{eV}$ \\
Angular Resolution & $9^{\circ} \mathrm{FWHM}$ \\
Dynamic Range & $10^{-4} \mathrm{~s}^{-1}$ to $80 \mathrm{~s}^{-1}$ (for Triple TOF Counts) \\
Time Resolution & Maps: $6 \mathrm{mo}$, features in ENA maps 3 mo \\
& ISN Flows: 1 year \\
SNR & ISN He $>10,000$, ISN H $>1000$, ISN O $>100$, ISN Ne, D $>10$, ISN Secondary \\
& He $>100$, Secondary O $>10$, ENA H (per pixel) $>10$, Expected average ENA H \\
\hline
\end{tabular}

\section{Instruments and Measurements}

IMAP provides comprehensive instrumentation to image the solar system's global interstellar boundaries and investigate the origins of particle acceleration (Table 1). This includes ENA instruments with average collection power increased by $\sim 15 \times, \sim 25 \times, \sim 35 \times$ for IMAP-Lo, IMAP-Hi, and IMAP-Ultra compared to IBEX-Lo, IBEX-Hi, and INCA (Fig. 5), improved, $4^{\circ}$, angular resolution, and the ability to resolve temporal changes down to 3 months. It also includes instruments optimized to measure interstellar atoms, suprathermal ions, energetic particles, and PUIs; a complete complement of solar wind proton, alpha, electron, and magnetic field measurements necessary for the next L1 real-time solar wind mission; interstellar UV glow maps from interstellar H and He; and ISD measurements. This section briefly summarizes the planned performance characteristics and designs of each IMAP instrument.

\subsection{IMAP-Lo}

IMAP-Lo, a single-pixel neutral atom imager mounted on a pivot platform, delivers (1) energy and angle-resolved measurements of ISN atoms $(\mathrm{H}, \mathrm{He}, \mathrm{O}, \mathrm{Ne}$, and $\mathrm{D})$ tracked over $>180^{\circ}$ in ecliptic longitude and (2) energy resolved global maps of ENA H and O. IMAPLo builds on the IBEX-Lo heritage (Fuselier et al. 2009b) but provides much larger collection power due to its position at L1 (average increased viewing time $4 \times$ due to low backgrounds) and increases in geometric factor $(3.2 \times)$, duty cycle, and efficiency. This maximizes the weak heliospheric neutral signal, while eliminating ion, electron, and UV background sources. Combined with the pivoting FOV, IMAP-Lo improves the angular resolution of ISN flow parameters. IMAP-Lo can track the ISN flow over almost the entire year, overcoming the serious limitations facing body-fixed sensors. IMAP-Lo's instrument performance parameters are summarized in Table 5.

IMAP-Lo, shown in Fig. 8, comprises collimation, conversion surface (CS), and time-offlight (TOF) subsystems; an electrostatic analyzer (ESA); electronics; a pivot platform; and a star sensor. With the pivot platform in its default position directing the FOV $90^{\circ}$ relative to the spin axis (pivot angle, $\mathrm{PA}=0^{\circ}$ ), IMAP-Lo views both ecliptic poles every spin, and completes a full-sky map every 6 months, as on IBEX. With PA $>0^{\circ}$, IMAP-Lo views a circular band with increased exposure at the equator and to maximal latitudes of $90^{\circ}-\mathrm{PA}$. 


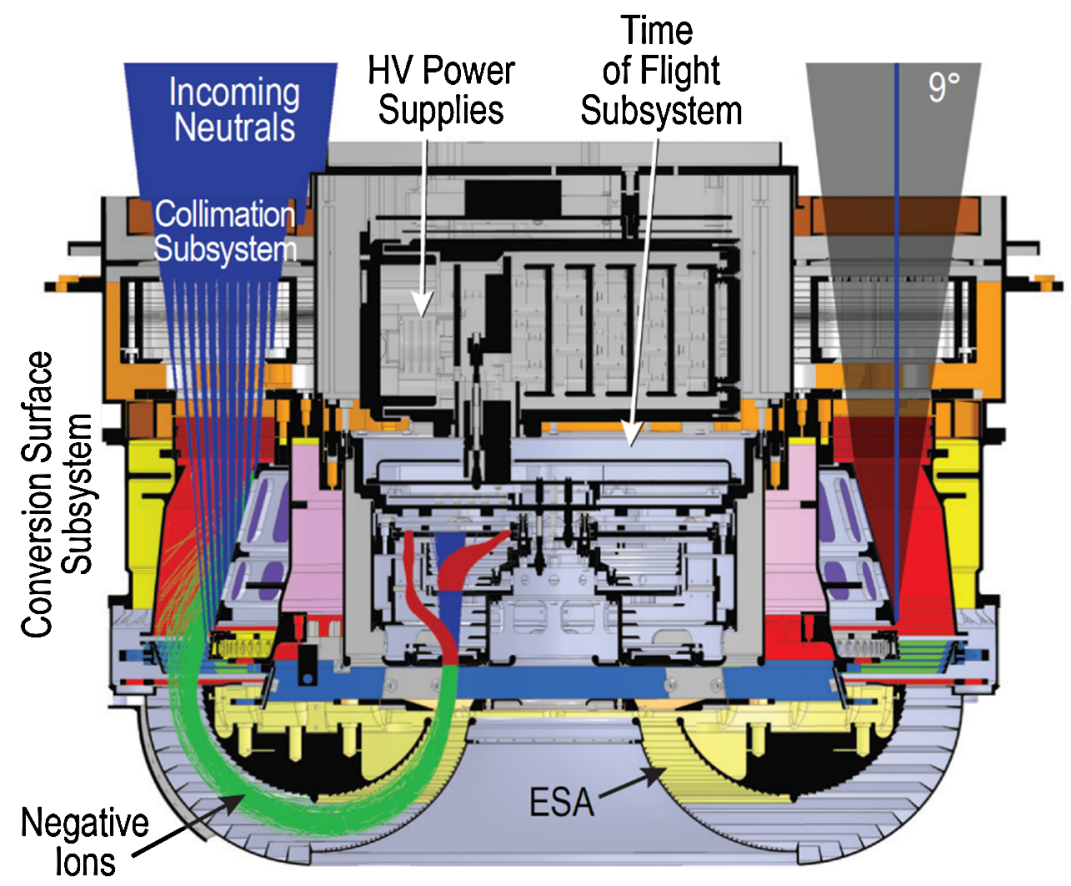

Fig. 8 IMAP-Lo cutaway drawing (right side), overlaid with SIMION ray-tracing (left side) of ENAs (blue), negative ions after conversion (green), neutrals and ions after passing the $\mathrm{C}$ foil(s) in the TOF section (blue), and secondary electrons emitted at the foils (red)

The pivoting FOV also enables IMAP-Lo to track the interstellar flow throughout the year and simultaneously optimize observations for global heliospheric imaging and observing the flow of ISN atoms.

ENAs enter through a collimator with a $9^{\circ}$ FWHM FOV (selected to resolve interstellar $\mathrm{H}, \mathrm{D}, \mathrm{He}, \mathrm{O}$, and $\mathrm{Ne}$ angular distributions with good statistics). Electrons $<600 \mathrm{eV}$ and ions $<3 \mathrm{keV}$ are rejected by a $4 \mathrm{keV}$ rejection electrode and the $+3 \mathrm{keV}$ collimator, respectively. ENAs strike a $15^{\circ}$ inclined diamond-like carbon CS on a Si wafer converting ENAs into negative ions (Wurz et al. 1997, 2006; Scheer et al. 2005, 2006; Wieser et al. 2007; Fuselier et al. 2009b; Bzowski et al. 2012; Möbius et al. 2009; Bochsler et al. 2012; Park et al. 2014; Funsten et al. 2009a). Resulting negative ions are pre-accelerated into a toroidal ESA, while secondary and photo-electrons are removed by a ring of magnets near the entrance of the ESA. The ESA's energy passband $(\Delta \mathrm{E} / \mathrm{E}=1)$, along with the wider FOV, yields a $3.2 \times$ increase of the geometric factor over IBEX-Lo including the energy passband. UV and scattered particles are rejected using a $\mathrm{Cu}$ black surface and 3-bounce design. After emerging from the ESA, ions are post-accelerated by $+16 \mathrm{kV}$ into the cylindrical triple-coincidence TOF subsystem. The first and second $\mathrm{C}$ foils $\left(\leq 1 \mu \mathrm{g} / \mathrm{cm}^{2}\right)$ produce secondary electrons, which are guided to the outer or inner annular start sections of the chevron configuration microchannel plate (MCP) detector. The ions continue to the central stop section of the MCP detector. Combining three independent TOF measurements reduces dark count rates to $\leq 1$ count/day (Möbius et al. 2008; Wurz et al. 2009).

Absolute pointing knowledge of IMAP-Lo to $\leq 0.1^{\circ}$ accuracy is achieved by a co-located star sensor identical in design to that on IBEX-Lo (Hlond et al. 2012; Fuselier et al. 2009b) but using a photomultiplier (PMT) with a cathode less sensitive in IR to reduce sensitivity 


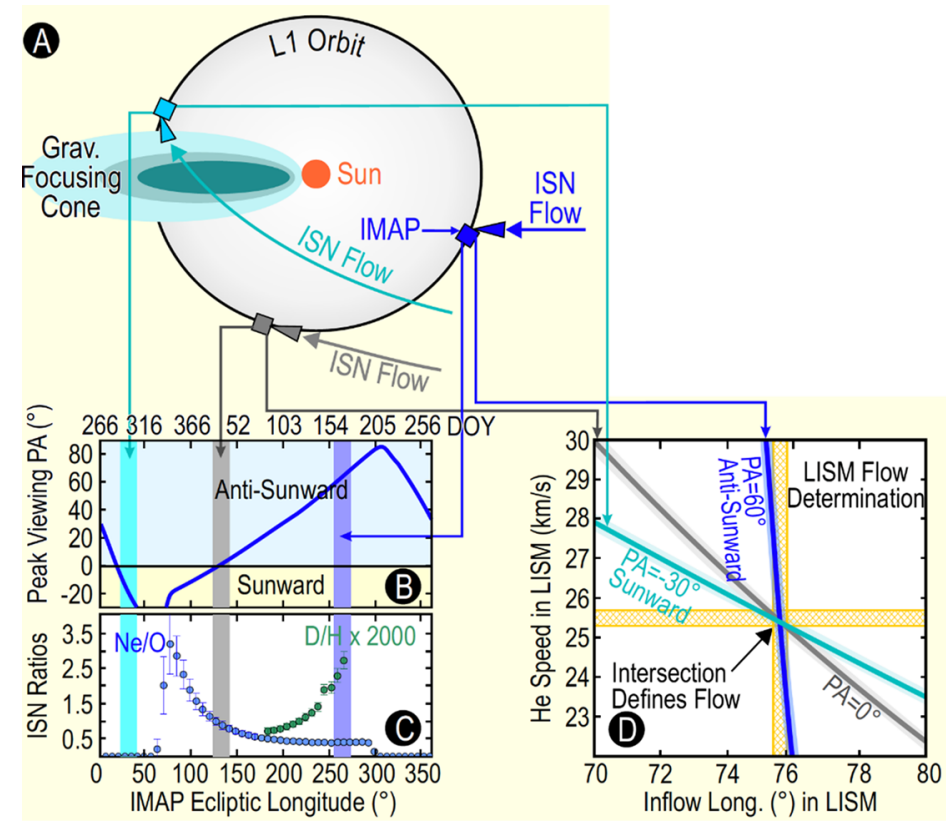

Fig. 9 IMAP-Lo tracks the interstellar flow to precisely determine the species-dependent flow speed, temperature, and direction of the LISM that surrounds, interacts with, and determines the outer boundaries of the global heliosphere. IMAP-Lo returns detailed abundance information defining composition of the LISM. The ecliptic longitude ( 3 cases shown along IMAP L1 orbit in top schematic), arrival direction relative to the Sun, and hyperbolic trajectory of the ISN bulk flow determine a characteristic relation between inflow longitude and speed at infinity. Panel $\mathbf{B}$ shows expected ISN flow direction relative to the Sun versus ecliptic longitude and IMAP Day of Year (DOY), including aberration due to orbital motion around the Sun, and panel $\mathbf{C}$ the expected $\mathrm{Ne} / \mathrm{O}$ and $\mathrm{D} / \mathrm{H}$ with statistical uncertainties for one orbit. IMAP-Lo uses the pivoting FOV (pivot angle, PA, with FOV $=90^{\circ}+\mathrm{PA}$ to spin-axis) to pinpoint the intersection in the relation between the ISN inflow speed and longitude to uniquely determine the LISM flow vector (panel D)

to Milky Way and Zodiacal Light background. The pivot platform orients the IMAP-Lo boresight up to $180^{\circ}$ from the spin axis, which is more than adequate to follow the interstellar flow through more than 180 days of the year (see Fig. 9). This also enables high counting statistics measurements of $\mathrm{Ne} / \mathrm{O}$ and $\mathrm{D} / \mathrm{H}$ (Fig. 9, panels B and $\mathrm{C}$ ). The varied orientation of the IMAP-Lo instrument also breaks an observational degeneracy due to the viewing restrictions for the interstellar flow with the fixed orientation of the IBEX-Lo FOV. This implies that each interstellar species detected by IBEX-Lo could be viewed at essentially the same time of year (see the grey point in panel A) or at essentially a single position along their trajectory, which results in a degenerate observation as a function of longitude. This produces a four-dimensional "parameter tube" (McComas et al. 2015a, 2015b; Swaczyna et al. 2015), where interstellar temperature, speed and latitude can all be expressed as a function of the interstellar inflow longitude (panel D). With IMAP-Lo, we measure the ISNs throughout the year using the pivot platform to view the bulk of inflowing neutral atoms (light blue, grey and dark blue points in panel A). At each position throughout the year, there is a distinct orientation of the parameter tube (light blue, grey and dark blue parameter tube in panel D). The intersection of these parameter tubes defines the inflow longitude of primary neutral atoms from the interstellar medium, and thereby breaks the measurement degeneracy faced by IBEX. 
Table 6 IMAP-Hi instrument performance

\begin{tabular}{ll}
\hline Parameter & Performance \\
\hline Instrument Type & 2 Independent Single-Pixel ENA Imagers \\
FOV & two $4^{\circ}$ FWHM, conical \\
& Hi-90_FOV centered $90^{\circ}$ to spin axis \\
& $4^{\circ}$ FWHM - FOV centered $45^{\circ}$ from spin axis \\
Angular Resolution & $0.41-15.6 \mathrm{keV}$ \\
Energy Range & $\leq 0.45\left(\mathrm{E}_{\mathrm{FWHM} / \mathrm{E})}\right.$ \\
Energy Resolution & 0.0008 at $0.5 \mathrm{keV}$ \\
GE $\left(\mathrm{cm}^{2}\right.$ sreV/eV) & 0.0024 at $1 \mathrm{keV}$ \\
& 0.04 at $10 \mathrm{keV}$ \\
SNR & $>100$ \\
Duty Cycle & 0.94 \\
Mass Resolution & $\mathrm{M} / \Delta \mathrm{M}=4(\mathrm{H}, \mathrm{He}, \mathrm{C} / \mathrm{N} / \mathrm{O} / \mathrm{Ne})$ \\
\hline
\end{tabular}

\subsection{IMAP-Hi}

IMAP-Hi consists of two identical, single-pixel high energy ENA Imagers that measure $\mathrm{H}$, $\mathrm{He}$, and heavier ENAs from the outer heliosphere. Each IMAP-Hi Imager is very similar in design to the IBEX-Hi ENA Imager (Funsten et al. 2009a) but incorporate key modifications that enable substantially improved (1) spectral, spatial, and temporal resolution, (2) spectral range, and (3) collection power. The instrument also incorporates time-of-flight (TOF) for identification of ENA species. These performance enhancements, yielding $\sim 25$ times the collection power of IBEX-Hi, enable unprecedented, detailed studies of structure and evolution of source plasmas in the heliosphere-LISM interaction region. The IMAP-Hi performance parameters are summarized in Table 6.

Each of the two identical IMAP-Hi imagers (Fig. 10) has four subsystems. An ENA first encounters the Entrance Subsystem, which consists of a pair of cylindrical deflection plates that reject ambient charged particles, and a grounded collimator, which consists of a series of parallel grids with co-aligned hexagonal apertures that defines the imager's $4^{\circ}$ FWHM near-conical FOV with a solid angle $\Omega_{\mathrm{FOV}} \sim 0.0038$ sr. The ENA then passes through the Charge Conversion Subsystem, which consists of over $150 \mathrm{~cm}^{2}$ of nominal $1 \mu \mathrm{g} / \mathrm{cm}^{2}$ carbon foils that convert a fraction of ENAs to positive ions (Funsten et al. 1993; McComas et al. 2004). Ionized ENAs then enter the Bundt-pan-shaped ESA Subsystem and enter the Detection Subsystem, which consists of inner and outer serrated plates shaped like a Bundt pan. They are independently biased to select a specific energy passband of ionized ENAs that can successfully pass to the Detector Subsystem, which consists of two sequential TOF stages that measure ENA speed. The combination of energy and speed measurements enables identification of ENA mass.

IMAP-Hi makes several key improvements compared to IBEX-Hi that enable superior performance. First, to remove charged particles before they can enter the collimator, the Entrance Subsystem utilizes bipolar $( \pm 5 \mathrm{kV})$, cylindrical deflection plates with ribs that prevent scattering of deflected ambient ions or electrons into the collimator. This enables IMAP-Hi to observe ENAs to $>15.6 \mathrm{keV}$ while eliminating the so-called "ion gun effect" that created some IBEX-Hi backgrounds when IBEX was in the magnetosheath and hot solar wind (Funsten et al. 2009a). Second, each ESA plate can be independently biased, enabling precise control of the energy passband central energy and width. Third, the Detector 


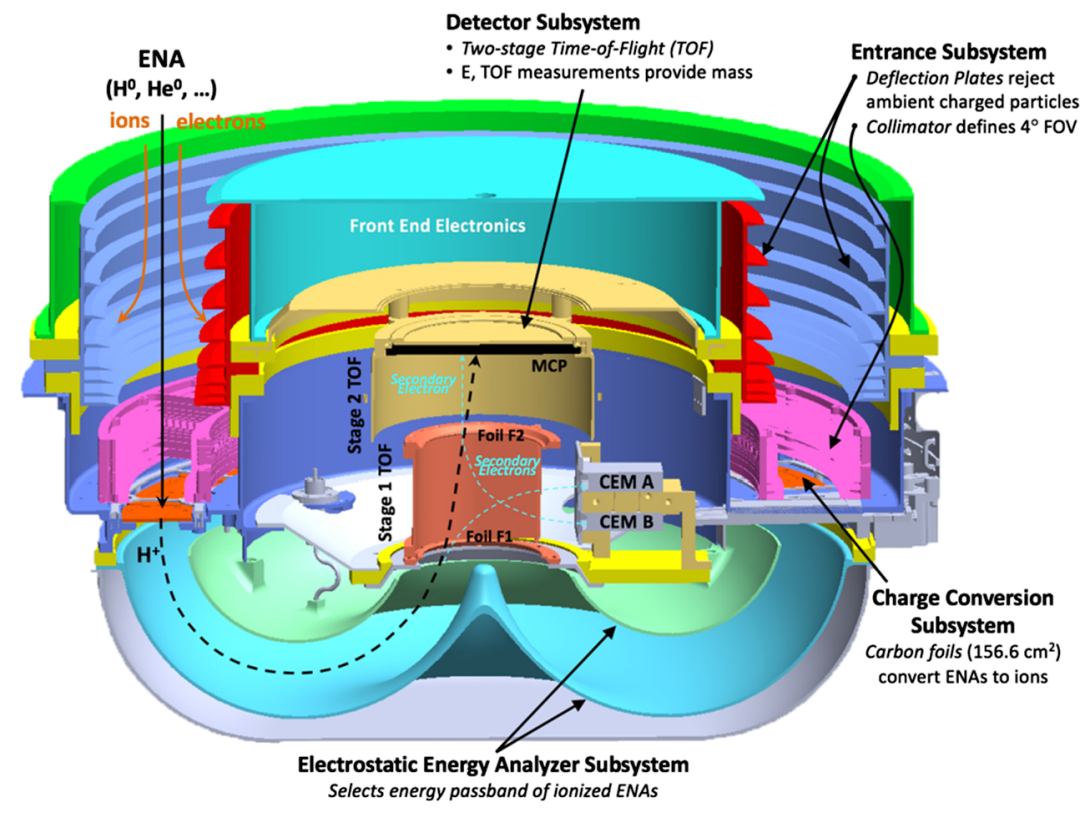

Fig. 10 Cross-sectional view of an IMAP-Hi Imager, with black trajectories that highlight the path followed by ENAs. Ambient ions and electrons are deflected before they enter the collimator

Fig. 11 Each IMAP-Hi imager has two TOF stages in series to provide species identification and to minimize noise. A prototype of the first TOF stage (Stage 1) designed after the HOPE mass spectrometer on Van Allen Probes (Funsten et al. 2013), shows well-resolved $\mathrm{H}, \mathrm{He}$, and heavy ions. This will provide unprecedented insight into the origin and dynamics of the ribbon and globally distributed ENA fluxes

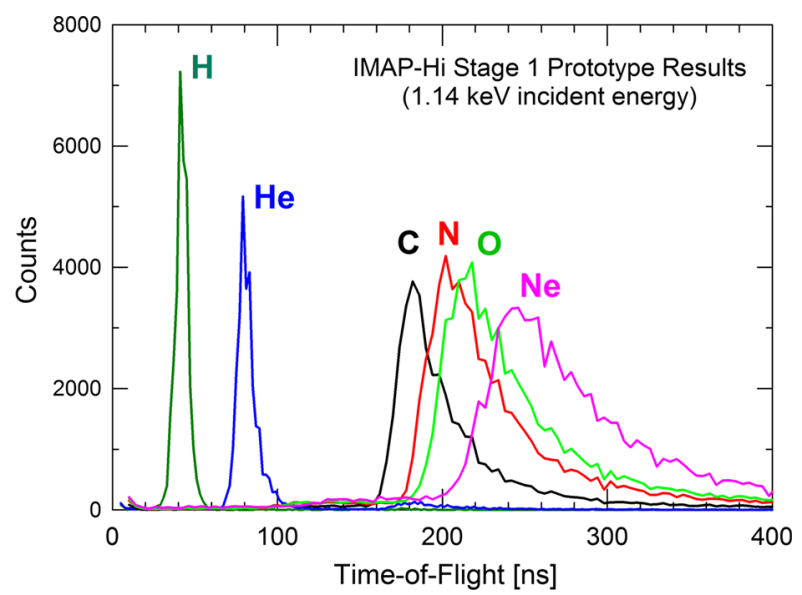

Subsystem consists of two linear, foil-based TOF stages; Stage 1 uses channel electron multiplier detectors and incorporates the electro-optic geometry developed and flown on Van Allen Probes/HOPE (Funsten et al. 2013), while Stage 2 uses a MCP detector to detect the primary ENA and secondary electrons from a carbon foil with multi-hit capability. A single ENA can generate up to four correlated events in Stages 1 and 2; event combinations are prioritized and reported, providing robust composition information and high noise rejection, especially from cosmic rays that generated significant background in the IBEX-Hi coincidence detector. Figure 11 shows the laboratory results of a TOF spectrum for a prototype of Stage 1, demonstrating the ability to separate $\mathrm{H}, \mathrm{He}$, and heavy ENAs. 


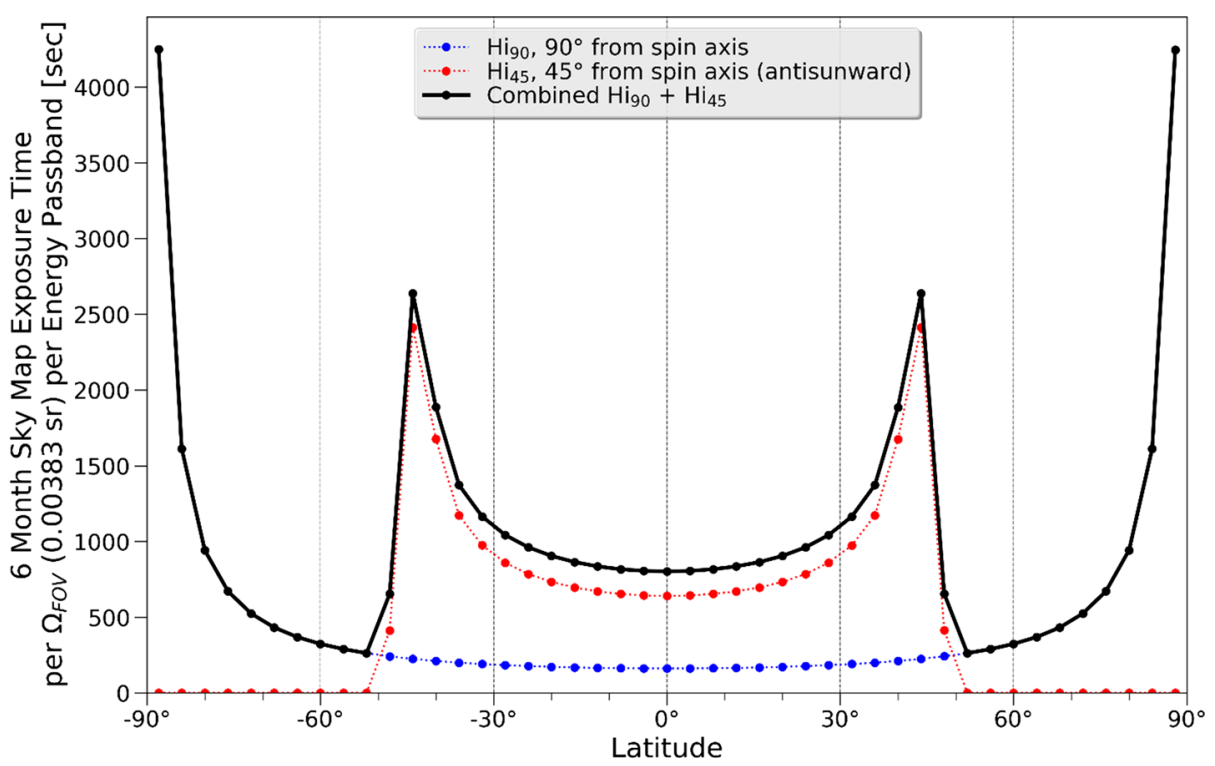

Fig. 12 The latitude dependence of the time exposure for each FOV pixel ( $\Omega_{\mathrm{FOV}} \sim 0.0038 \mathrm{sr}$ ) for a 6-month sky map at each energy passband is shown for the $\mathrm{Hi}_{45}$ imager (red line), Hig0 imager (blue line), and combined $\mathrm{Hi}_{45}$ and $\mathrm{Hi}_{90}$ imagers (black line). The Hig0 imager covers the entire sky each map, while the $\mathrm{Hi}_{45}$ imager provides extended viewing of the lower latitude emission regions that are under sampled by the Higo imager and includes the nose, heliotail, and a majority of the ribbon and belt

The two IMAP-Hi imagers are mounted $90^{\circ}\left(\mathrm{Hi}_{90}\right.$ imager $)$ and $45^{\circ}\left(\mathrm{Hi}_{45}\right.$ imager $)$ antisunward relative to the spacecraft spin axis, respectively. Each spin, the $\mathrm{Hi}_{90}$ imager views the ecliptic poles, while the combined $\mathrm{Hi}_{90}$ and $\mathrm{Hi}_{45}$ imagers sample the ecliptic plane in four different locations. Also, the $\mathrm{Hi}_{90}$ and $\mathrm{Hi}_{45}$ imagers enable viewing the same low-latitude regions twice within 3 months to probe rapid ENA time variability. As shown in Fig. 12, the $\mathrm{Hi}_{45}$ imager has increased exposure of a factor of $\times 3.9$ at the equator and nearly $\times 10$ at $45^{\circ}$ latitude, a band that contains ENA emission from the heliospheric nose and tail, as well as most of the Ribbon and Belt.

\subsection{IMAP-Ultra}

The IMAP-Ultra instrument (Ultra) images the emission of ENAs produced in the heliosheath and beyond, primarily in $\mathrm{H}$ atoms between $\sim 3$ and $300 \mathrm{keV}$, but also sensitive to contributions from He and O. Ultra is nearly identical to the Jupiter Energetic Neutral Imager (JENI; Mitchell et al. 2016), in development for flight on the European Space Agency's JUICE mission to Jupiter and Ganymede. Ultra's primary differences from JENI include (1) the use of 2 identical copies, one mounted perpendicular to the IMAP spin axis (Ultra 90 ) and one mounted at 45 degrees from the anti-sunward spin axis (Ultra 45 ) for better sky coverage and more uniform sensitivity over the heliosphere, similar to IMAP-Hi, and (2) the use of slightly thicker, UV-filtering foils covering the back plane MCPs to reduce backgrounds associated with interstellar Lyman- $\alpha$ photons. High resolution images covering over $50 \%$ of the heliosphere are produced every $\sim 3$ months, contributing to the understanding of the particle spectra and pressures in and beyond the heliosheath, and detecting changes in the spatial distribution of those particles on time scales sufficient to track both solar cycle as 
Table 7 IMAP-Ultra Instrument Performance

Fig. 13 IMAP-Ultra cutaway showing major elements: charged-particle deflection plates; front MCP assembly with two 1D start anodes servicing two entrance slits, and one 1D coincidence anode; variable aperture mechanisms servicing each entrance slit (detailed in inset); two stop MCPs imaged using 2D anodes; and a strip of SSD detectors between the two stop MCPs

\begin{tabular}{|c|c|}
\hline Parameter & Performance \\
\hline Instrument Type & Slit-Based ENA Imager \\
\hline Species & Neutrals, ions, $\mathrm{e}^{-}$ \\
\hline FOV & $90^{\circ} \times 120^{\circ}(\mathrm{MCPs}) ; 70^{\circ} \times 120^{\circ}(\mathrm{SSDs})$ \\
\hline Angular Resolution & $2^{\circ}(\mathrm{H}>10 \mathrm{keV}) ; 10^{\circ}(\mathrm{O}>30 \mathrm{keV})$ \\
\hline Energy Range & $\begin{array}{l}3-300 \mathrm{keV}(\mathrm{H}), 5 \mathrm{MeV} \text { (ions) [TOF-only]; } \\
35-x(\mathrm{H}), 60-x(\mathrm{He}), 0.08-x(\mathrm{O})[T O F \times E] \\
\text { where } x=300 \mathrm{keV} / \mathrm{q} \text { neutrals, } 5 \mathrm{MeV} \text { ions; } \\
30-700 \mathrm{keV} \text { (electrons, SSD-only) }\end{array}$ \\
\hline Energy Resolution & $\leq 14 \%(>10 \mathrm{keV})$ \\
\hline$G F\left(\mathrm{~cm}^{2} \mathrm{sr}\right)$ & $\begin{array}{l}0.012-0.31 \text { (high res, low background) } \\
1.22 \text { (high sensitivity) }\end{array}$ \\
\hline SNR & Function of energy: $\sim 10$ to 1000 \\
\hline Mass Resolution & $\begin{array}{l}\text { MCP-based: } \mathrm{H} \text { (lights), } \mathrm{O} \text { (Heavies) } \\
\text { SSD-based: } \mathrm{H}, \mathrm{He}, \mathrm{O}, \mathrm{Ne}, \mathrm{Fe}(\mathrm{M} / \Delta \mathrm{M} \sim 2)\end{array}$ \\
\hline
\end{tabular}

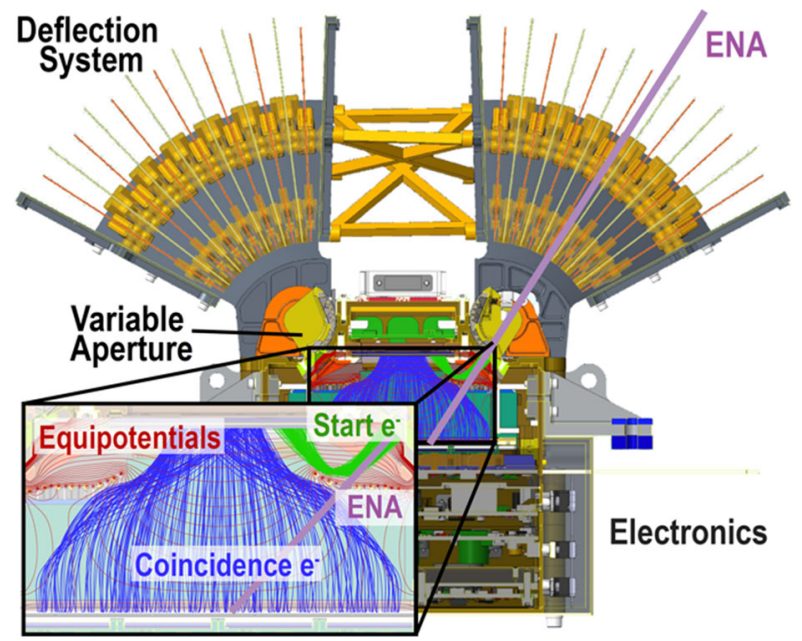

well as major active region dependencies. Ultra's performance parameters are summarized in Table 7.

Each Ultra (Fig. 13) has two identical entrances composed of a deflection system, variable aperture (VA), slit, and start foil. Particles (Fig. 13, purple trajectory) enter the deflection system, where high voltage (HV) plates deflect the charged component into the serrated plate surfaces, rejecting ions (electrons) by factors of $\sim 10^{4}(\sim 100)$ below $300 \mathrm{keV} / \mathrm{q}$, allowing ENAs access to the sensor. The VA provides choices for sensitivity, angular resolution, and UV filtering. The ENA penetrates the Start foil, providing a position and start time for measuring TOF. The ENA continues to a stop foil, penetrates that, and hits the stop MCP where a stop position, stop time, and MCP pulse height are recorded. Secondary electrons from the impact side of the stop foil are guided to a coincidence area on the front MCP, providing a position that must match the stop position, as well as a tight timing window of $\sim 4$ ns. These coincidence criteria greatly reduce backgrounds from UV and cosmic rays. 
Table 8 MAG instrument performance

\begin{tabular}{ll}
\hline Parameter & Performance \\
\hline Instrument Type & Vector Triaxial Fluxgate Magnetometer \\
Sample Rates & $2 \mathrm{~Hz} ; 128 \mathrm{~Hz}$ \\
Dynamic Range & $\pm 500 \mathrm{nT}$ \\
Resolution & $10 \mathrm{pT}$ \\
Zero-Level Stability & $<0.1 \mathrm{nT}$ baseline drift per month \\
\hline
\end{tabular}

In this way the sensor measures the velocity, trajectory and pulse heights for the ENA, and bins the event appropriately in an accumulated image.

Ultra achieves $<2^{\circ}$ angular resolution for $>10 \mathrm{keV} \mathrm{H}$, degrading to $\sim 8^{\circ}$ angular resolution at $3 \mathrm{keV}$. For a given measured velocity, species (primarily $\mathrm{H}$ and $\mathrm{O}$ ) are determined from the Stop MCP pulse height. Above $\sim 30 \mathrm{keV}$ for $\mathrm{H}$, and $100 \mathrm{keV}$ for O, Ultra provides more definitive composition using its strip of solid state detectors (SSDs) to measure the ion energy together with its velocity.

Ultra will complement the measurements of IMAP-Lo and IMAP-Hi, providing overlap in energy with IMAP-Hi for heliospheric energetic $\mathrm{H}$, with comparable or better angular resolution, and extending the measurements through the range covered previously by Cassini/INCA with much improved sensitivity, angular resolution, and background rejection (see panels $\mathrm{C}$ and $\mathrm{F}$ in Fig. 5).

\subsection{MAG}

The IMAP magnetometer (MAG) consists of a pair of identical triaxial fluxgate magnetometers based on the Magnetospheric Multiscale (MMS) magnetometers (Russell et al. 2016), for which UCLA built 4 electronics units and 9 fluxgate sensors. On IMAP, the magnetometers are both mounted on a $1.8 \mathrm{~m}$ boom to reduce the impact of the magnetometer on spacecraft design, and through gradiometry to reduce the effect of spacecraft magnetic fields on the measurements of the instrument by dynamically removing the spacecraft field. This technique was tested and proven valuable on the Venus Express mission with only a $1 \mathrm{~m}$ boom and no magnetic cleanliness program (Russell et al. 2013a). Even the signals of the Venus Express spinning magnetic reaction wheels were removed. The gradiometer not only maintains an accurate baseline for space weather applications, but also allows new insight into waves and turbulence in the solar wind to frequencies near the electron gyrofrequency. MAG's performance parameters are summarized in Table 8 .

Each magnetometer resembles the complete assembly with cable shown in the inset in Fig. 14. Assembly is straightforward with the main frame bottom supporting the cable and triaxial sensor. The sensor's printed circuit board sits in the main frame bottom and the Z core assembly lies on top of the board. The XY core assembly is a single core wrapped to measure two components ( $\mathrm{X}$ and $\mathrm{Y}$ ) of the magnetic field. The assembly is capped with the main frame top which also contains a second printed circuit board. The three orthogonal pairs of nulling coils wrap around the outside of the main frame. This sensor design has been successfully used for nearly 4 years in space at this writing on 8 instruments on the 4 MMS spacecraft.

The magnetometer uses sigma-delta digitization integrated in the measurement loop to generate values along each axis that are sent continuously to the processor board. The processor provides two output streams, one $128 \mathrm{~Hz}$ for scientific studies of the local magnetized plasma and one at $2 \mathrm{~Hz}$ to serve the real-time space weather monitoring system. The 
Fig. 14 Major components and assembly of MAG

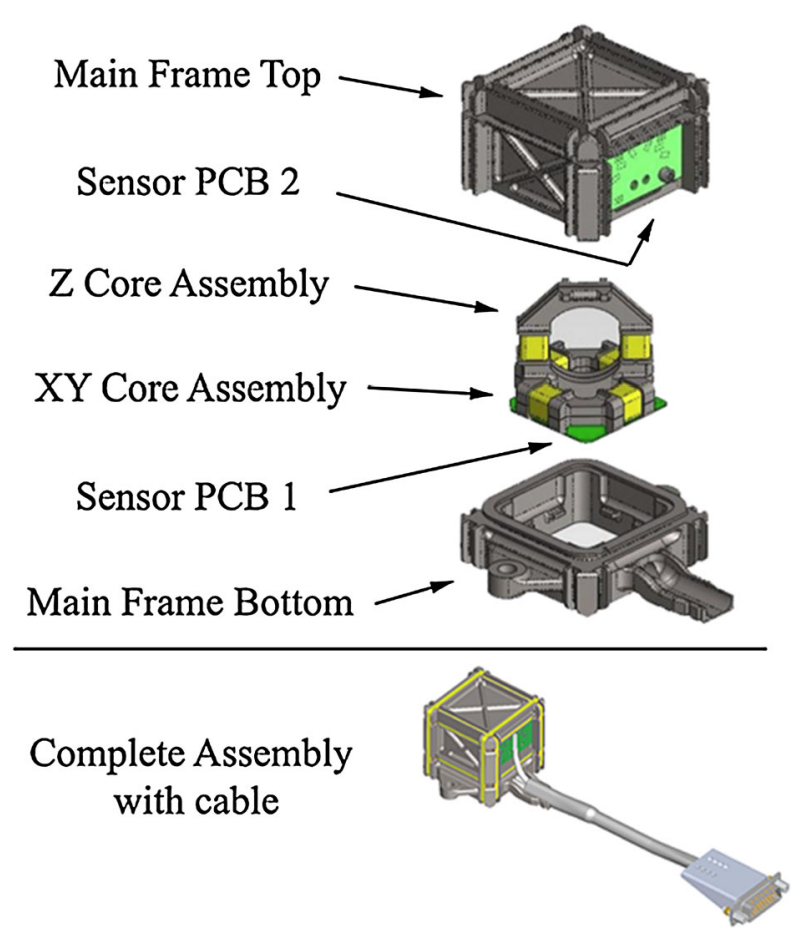

dynamic range is $\pm 500 \mathrm{nT}$ that easily envelops the largest interplanetary magnetic field ever recorded (Russell et al. 2013b) and are returned to Earth as three 24 bit components. The high stability of the zero levels of these cores together with the real-time operation in the Alfvenic fluctuations in the solar wind allow the baseline zero level to be maintained to $\pm 0.1 \mathrm{nT}$.

\subsection{SWE}

The Solar Wind Electron (SWE) instrument measures the 3D distribution of SW thermal and suprathermal electrons from $1 \mathrm{eV}$ to $5 \mathrm{keV}$. SWE is based on the heritage Ulysses/SWOOPS (Bame et al. 1983, 1992), ACE/SWEPAM (McComas et al. 1998) and Genesis/GEM (Barraclough et al. 2003) instruments, with updated electronics based on Van Allen Probes/HOPE (Funsten et al. 2013). SWE is optimized to measure in situ solar wind electrons at L1 to provide context for the ENA measurements and perform the in situ solar wind observations necessary to understand the local structures that can affect acceleration and transport. SWE instrument performance parameters are summarized in Table 9.

SWE consists of a spherical section ESA followed by seven channel electron multiplier (CEM) detectors (Fig. 15). Electrons enter through an aperture pointed normal to the spacecraft spin axis. A positive HV on the inner ESA plate biases the analyzer such that only electrons within a narrow range of energies and azimuthal angles pass through and are detected by the CEMs. Stepping this voltage provides coverage of the full energy range. Electrons entering the instrument at different angles relative to the aperture normal are detected in different CEMs, yielding $21^{\circ}$ polar angle resolution within the fan-shaped FOV. As the IMAP spacecraft spins, the SWE fan-shaped FOV sweeps out $>95 \%$ of $4 \pi \mathrm{sr}$, missing only small conical holes centered parallel and antiparallel to the spin axis. The curved-plate ESA is 
Table 9 SWE instrument performance

\begin{tabular}{ll}
\hline Parameter & Performance \\
\hline Instrument Type & SW Electron ESA \\
FOV & $160^{\circ} \times 12^{\circ}$ instantaneous \\
Angular Range \& & Polar angle range $10-170^{\circ}, 21^{\circ}$ resolution \\
Resolution & Azimuthal range $0-360^{\circ}$ over spin, $\sim 12^{\circ}$ \\
& intrinsic resolution (varies w/ polar angle) \\
Energy Range & $0.001-5 \mathrm{keV}$ \\
Energy Resolution & $12 \%$ \\
$G E\left(\mathrm{~cm}^{2}\right.$ sreV/eV) & $2-7 \times 10^{-4}$ per pixel \\
Dynamic Range & $\mathrm{Up} \mathrm{to} 500 \mathrm{kHz}$ \\
Time Resolution & $15 \mathrm{~s}$ \\
\hline
\end{tabular}

composed of nested $120^{\circ}$ spherical grooved and blackened $\mathrm{Al}$ alloy plates. The plates have a gap width of $3.5 \mathrm{~mm}$ and an average radius of $41.9 \mathrm{~mm}$.

SWE measurements are made by each of the seven CEM detectors as a function of spin angle and energy step. The $\sim 12 \%$ energy resolution and $\sim 12^{\circ} \times 21^{\circ}$ angular resolution allow full characterization of the solar wind electron distributions, including moments and energy and angle distributions of the thermal, suprathermal, and strahl populations. The extension of the energy range to $5 \mathrm{keV}$ also permits characterization of the superhalo population. SWE, together with SWAPI and MAG, also provide 1 AU context for IMAP ENA observations, including measurement of solar wind structures and dynamics.

\subsection{SWAPI}

The Solar Wind and Pickup Ion (SWAPI) instrument measures solar wind $\mathrm{H}^{+}$and $\mathrm{He}^{++}$ and interstellar $\mathrm{He}^{+}$and $\mathrm{H}^{+}$PUIs. SWAPI is nearly identical to the NH Solar Wind Around Pluto (SWAP) instrument (McComas et al. 2008), which has over a decade of flight-proven observations of solar wind and PUI distributions (e.g., McComas et al. 2017b). SWAPI is a simplification of SWAP, and by removal of SWAP's retarding potential analyzer, significantly increases transmission and improves sensitivity, further enhancing PUI observations.

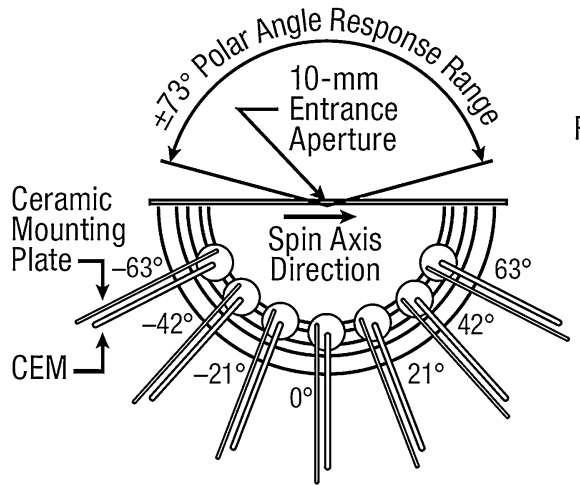

Back View

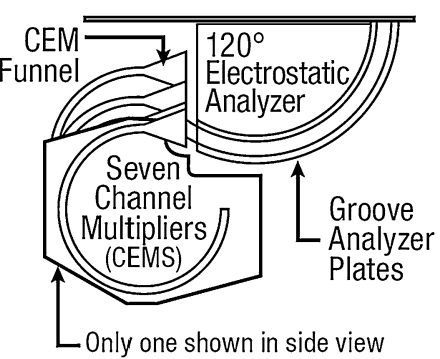

Cross-Sectional Side View

Fig. 15 IMAP-SWE schematic 
Table 10 SWAPI instrument performance

Fig. 16 Cross-sectional view of the SWAPI sensor with blue trajectories that highlight the incident solar wind and green that highlight PUIs. Yellow trajectories show the path of the secondary electrons generated from the foil to provide the coincidence timing

\begin{tabular}{ll}
\hline Parameter & Performance \\
\hline Instrument Type & SW/PUI Spectrometer ESA \\
Species & SW $\mathrm{H}^{+}$and $\mathrm{He}^{++}$; Interstellar $\mathrm{He}^{+}$and $\mathrm{H}^{+}$ \\
FOV & Sun-viewing: $20^{\circ} \times 10^{\circ}$ \\
& Non-Sun-viewing: $256^{\circ} \times 10^{\circ}$ \\
SNR & Min. SNR of 1 @ $0.07 \mathrm{~Hz}$ coincident count rate \\
Energy Range & $0.1-20 \mathrm{keV} / \mathrm{q}$ \\
Energy Resolution & $8.5 \%$ \\
GE $\left(\mathrm{cm}{ }^{2}\right.$ sreV/eV) & SW Sun-viewing: $1.1 \times 10^{-7}$ for SW protons \\
& PUI Non-Sun-viewing: $1.6 \times 10^{-2}$ for PUI He \\
Dynamic Range & $2 \mathrm{MHz}$ to $0.07 \mathrm{~Hz}$ counting rates \\
Time Resolution & $15 \mathrm{~s}$ \\
\hline
\end{tabular}

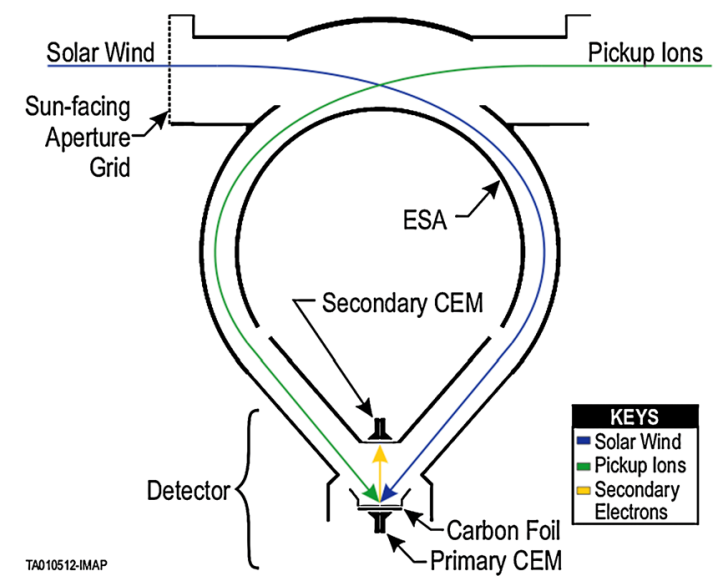

Detailed measurements of these populations are fundamental to understanding the solar wind context, sources and acceleration of particles, PUIs, and the physical processes regulating the global heliosphere. SWAPI delivers the high time and energy resolution required to identify local acceleration processes. SWAPI's instrument performance parameters are summarized in Table 10.

The SWAPI electro-optics (Fig. 16) consists of a grounded aperture grid that attenuates the solar wind beam without reducing PUIs from all other directions, an ESA for energyper-charge (E/q) selection, a field-free flight path, and a coincidence detector section. These elements select the energies and separate geometric factors for solar wind and PUIs. Transmitted ions are post-accelerated into the detector section that employs an ultrathin carbon foil (McComas et al. 2004) and two CEMs to make a coincidence measurement when both the primary particle and front-side secondary electrons are detected within a $100 \mathrm{~ns}$ window.

By properly masking the sunward direction, SWAPI makes critical measurements of the solar wind conditions at $1 \mathrm{AU}$, including $\mathrm{H}^{+}, \mathrm{He}^{++}$, and heavier ions during cold solar wind conditions such as in many interplanetary coronal mass ejections (ICMEs). Away from the solar wind direction, there is no aperture reduction, increasing PUI sensitivity by $5 \times$ compared to SWAP. The SWAPI energy range is extended up to $20 \mathrm{keV} / \mathrm{q}$ to capture the $1 \mathrm{D} \mathrm{He}^{+}$and $\mathrm{H}^{+}$PUI distributions and cutoffs, as well as both the solar wind $\mathrm{H}^{+}$and $\mathrm{He}^{++}$ 
Fig. 17 PUI and solar wind counts and uncertainties for $15 \mathrm{~s}$ (grey) and $60 \mathrm{~s}$ (green) accumulation times, scaled through a model from actual SWAP observations at $26 \mathrm{AU}$. SWAPI will accurately measure the broader solar wind $\mathrm{H}^{+}$and $\mathrm{He}^{++}$peaks and the $\mathrm{He}^{+}$PUIs above the $\mathrm{H}^{+}$PUI cutoff

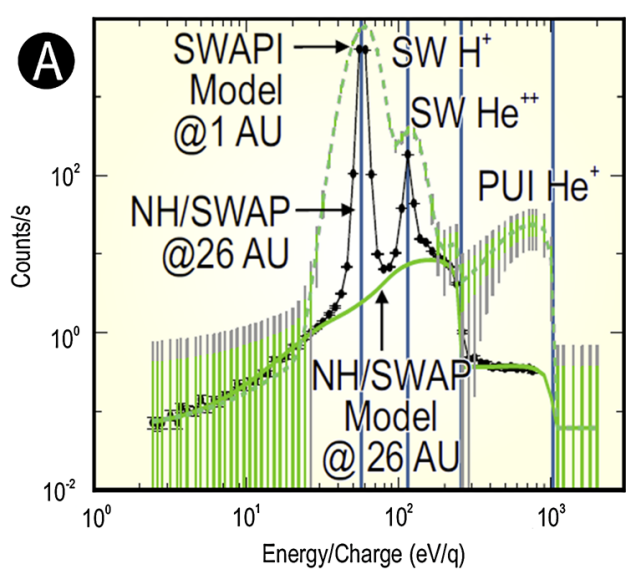

distributions. In addition to providing outstanding solar wind observations, SWAPI's rapid measurements of PUI $\mathrm{He}^{+}$enable critical new understanding of ion pickup and acceleration processes. Figure 17 shows an example of a coincidence sweep of SWAP observations at $\sim 26 \mathrm{AU}$ (black) and modeled measurements at $1 \mathrm{AU}$ from SWAPI (green/grey).

\subsection{CoDICE}

The Compact Dual Ion Composition Experiment (CoDICE) combines an ESA with a common TOF versus energy (TOF/E) subsystem to simultaneously measure (1) the 3D velocity distribution functions (VDFs) and ionic charge state and mass composition of $\sim 0.5-80 \mathrm{keV} / \mathrm{q}$ ions in $\mathrm{CoDICE}_{L o}$, and (2) the mass composition and arrival direction of $\sim 0.03-5 \mathrm{MeV} /$ nuc ions in $\mathrm{CoDICE}_{H i}$ (Desai et al. 2015, 2016b, 2017). CoDICE $\mathrm{Hi}_{\mathrm{i}}$ also measures $\sim 20-600 \mathrm{keV}$ electrons. These measurements, summarized in Table 11, are required to (1) determine the LISM composition and flow properties and (2) discover the origin of the enigmatic suprathermal tails and advance understanding of the acceleration of particles in the heliosphere. Figure 18 shows the overall design of CoDICE.

$\mathrm{CoDICE}_{L o}$ measures ions between $\sim 0.5$ and $80 \mathrm{keV} / \mathrm{q}$ that enter the collimated aperture (blue trajectories in Fig. 18) and are selected and focused according to their E/q into the Start TOF subassembly. The E/q sweeping steps up to the maximum voltage for the next stepping cycle when solar wind $\mathrm{H}^{+}$count rates near $\sim 1 \mathrm{keV} / \mathrm{q}$ exceed a predefined threshold rate. The Start subassembly, biased at $-15 \mathrm{kV}$, accelerates the incoming ions before they strike a thin carbon foil $\left(\sim 1 \mu \mathrm{g} / \mathrm{cm}^{2}\right)$ at the entrance of the TOF/E subsystem. This interaction produces secondary electrons (red trajectories) that are guided to the outer annulus of the Start MCP. The mostly neutralized ions (black trajectories) traverse a $9.7 \mathrm{~cm}$ flight path and strike one of 24 Avalanche Photodiodes (APDs; Ogasawara et al. 2016) where they generate secondary electrons that are guided to the center of the Stop MCP (purple trajectories). The TOF between the Start and Stop MCP signals yields the ion velocity. The residual energy (E) of the neutralized ion is measured by one of the 24 azimuthally distributed APDs that collectively provide $6^{\circ} \times 360^{\circ}$ instantaneous FOV and cover $\sim 2.7 \pi \mathrm{sr}$ in a spacecraft spin. The combined measurements, E/q, TOF, and E, determine the mass (M), charge state $(\mathrm{q})$, and $\mathrm{M} / \mathrm{q}$.

$\mathrm{CoDICE}_{\mathrm{Hi}}$ measures ions between $\sim 0.03$ and $5 \mathrm{MeV} /$ nuc (orange trajectories) that enter the common TOF/E subsystem through 12 separate $12^{\circ} \times 7^{\circ}$ FOV collimators and $100 \mathrm{~nm}$ 


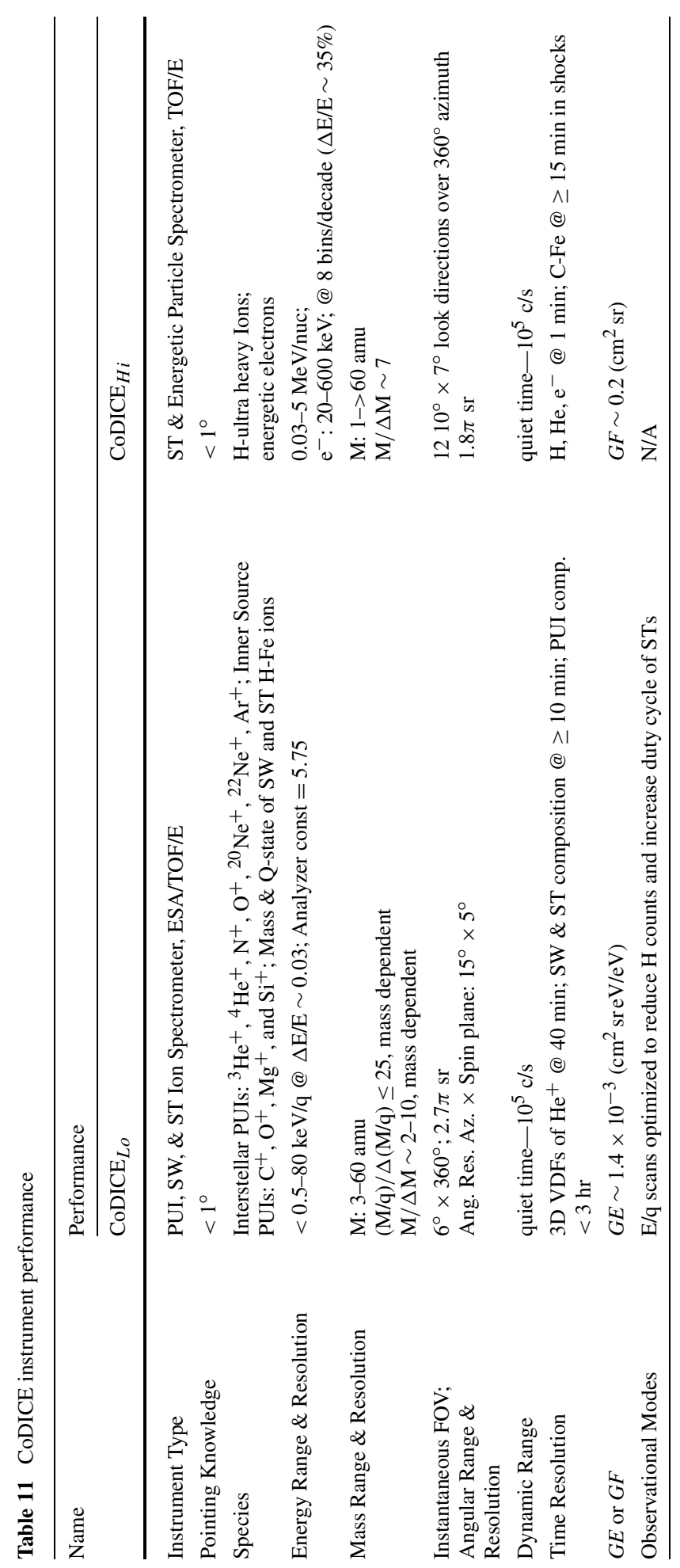


Fig. 18 CoDICE cross-section showing electro-optical design and simulations of particle trajectories through the ESA and $\mathrm{TOF} / \mathrm{E}$ subsystems

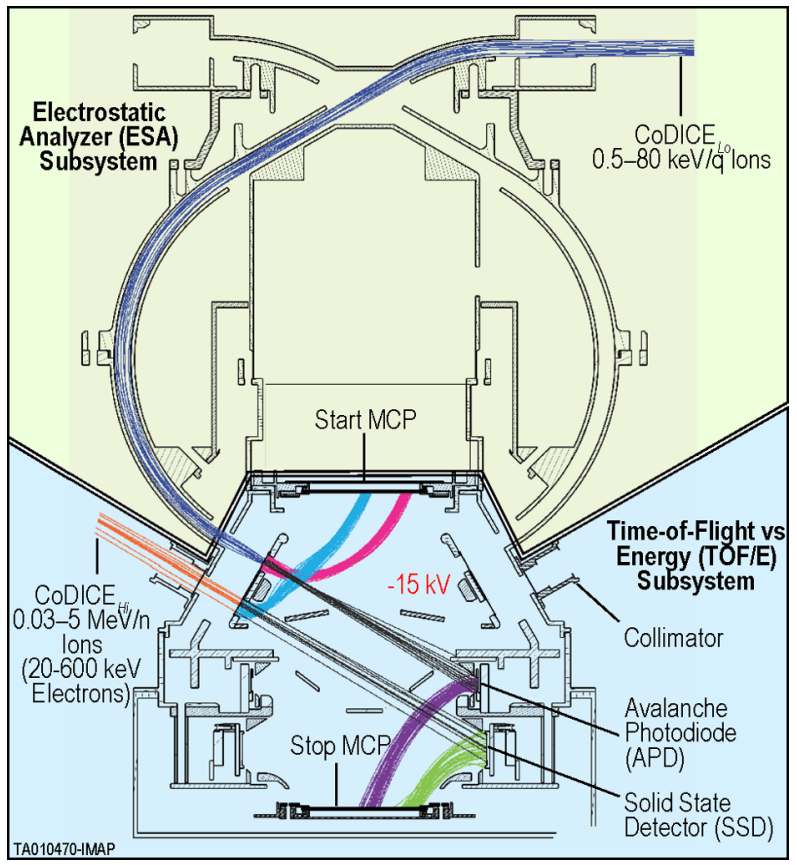

Al-C-Si foils that attenuate UV light to $<0.1 \%$ and stop low-energy $(<10 \mathrm{keV})$ protons. Start and Stop MCP signals are generated by secondary electrons (light blue and green trajectories) that are produced as the ions traverse a thin $\sim 1 \mu \mathrm{g} / \mathrm{cm}^{2} \mathrm{C}$ foil located at the $\mathrm{TOF} / \mathrm{E}$ entrance and strike one of $12 \mathrm{SSDs}$ distributed over $360^{\circ}$ azimuth, respectively. Each $\mathrm{SSD}, 700 \mu \mathrm{m}$ thick with an active area of $15 \times 15 \mathrm{~mm}^{2}$, determines the ion's E. This, along with the TOF, yields the ion velocity and provides the ion mass. The secondary electrons are detected by the same MCPs shared with $\mathrm{CoDICE}_{L o}$, but in separate areas; Hi-Start electrons (light blue trajectories) are guided toward the center portion of Start MCP, and Hi-Stop electrons (green trajectories) are guided toward the outer annulus of Stop MCP. Electrons $>20 \mathrm{keV}$ are identified by deposited energy and $<2$ ns TOFs. In addition, two partially segmented SSDs at $45^{\circ}$ to Sun-Earth line are flashed with Al to block low-energy ions. CoDICE $_{H i}$ covers $1.8 \pi$ sr in each spacecraft spin.

Figure 19 summarizes the prototype test results for $<300 \mathrm{keV}$ ion beams, confirming the power-law relationship between TOF and $\mathrm{E}$ and validating CoDICE performance for species separation.

\subsection{HIT}

The High-energy Ion Telescope (HIT) measures the elemental composition, energy spectra, angular distributions, and arrival times of $\mathrm{H}$ to $\mathrm{Ni}$ ions over a species-dependent energy range from $\sim 2$ to $\sim 40 \mathrm{MeV} /$ nuc. HIT, heavily based on the Low Energy Telescope (LET) on the Solar Terrestrial Relations Observatory (STEREO) (Mewaldt et al. 2008), delivers full-sky coverage from a large GF instrument to enable 1 min resolution of ion anisotropy measurements, observing the ramps of local shocks, anchoring the high-energy SEP ion spectra, and resolving particle transport in the heliosphere. HIT's instrument performance parameters are summarized in Table 12. 

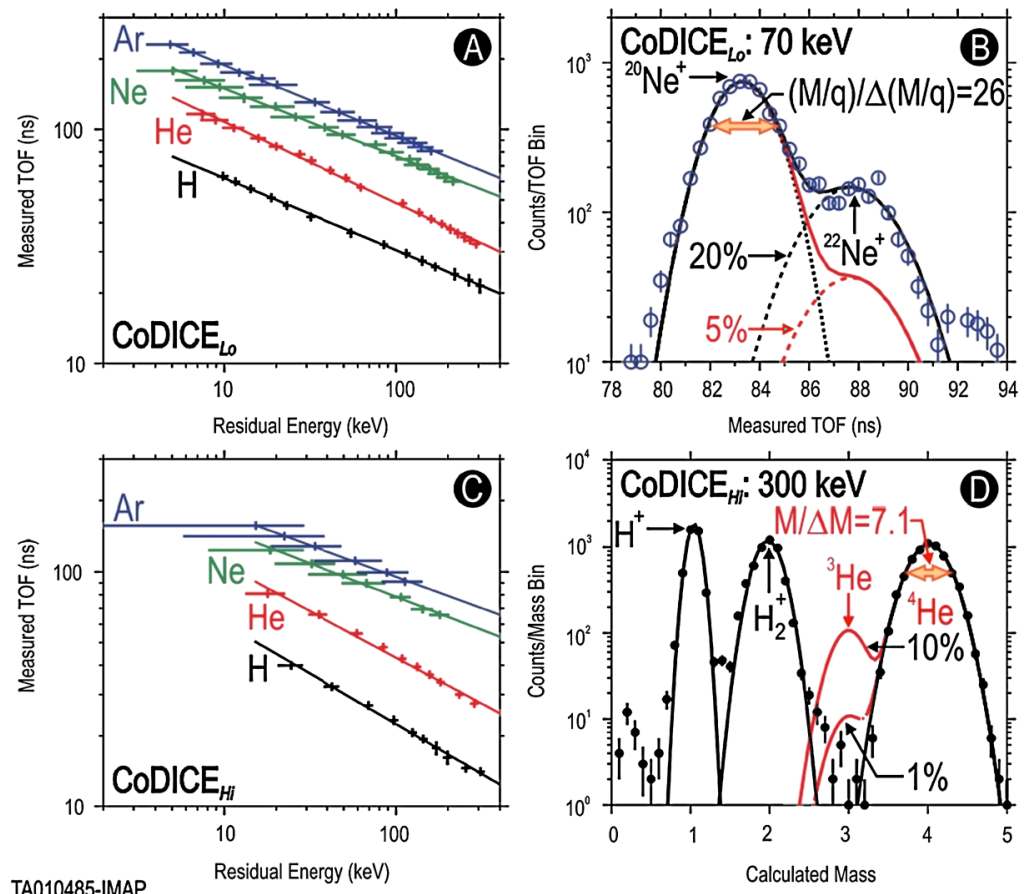

Fig. 19 The CoDICE prototype performance demonstrates the expected TOF/E relationship (A, C) and excellent M/Q and $\mathrm{M}$ resolution $(\mathbf{B}, \mathbf{D})$ for $\mathrm{CoDICE}_{L o}$ and $\mathrm{CoDICE}_{H i}$, respectively. $\mathrm{CoDICE}_{L o}$ APDs achieve excellent energy resolution at residual energies below $10 \mathrm{keV}$, enabling triple-coincidence measurements in all types of solar wind flow speeds with only $-15 \mathrm{kV}$ bias for post-acceleration. (B, D) $\operatorname{CoDICE}_{L o}$ has $\mathrm{M} / \mathrm{Q} / \Delta(\mathrm{M} / \mathrm{Q}) \sim 26$, allowing separation of all major and rare interstellar PUI species and isotopes $\left({ }^{22} \mathrm{Ne}^{+}\right.$ and ${ }^{3} \mathrm{He}^{+}$). With $\mathrm{M} / \Delta \mathrm{M} \sim 7$, CoDICE $\mathrm{Hi}$ resolves ${ }^{3} \mathrm{He}$ from ${ }^{4} \mathrm{He}$ even when the corresponding ratio falls below $\sim 10 \%$, as it often does in large SEP events $(\mathbf{D})$

Table 12 HIT instrument performance

\begin{tabular}{ll}
\hline Parameter & Performance \\
\hline $\begin{array}{l}\text { Instrument Type } \\
\text { Species }\end{array}$ & $\begin{array}{l}\text { Energetic Particle Spectrometer, dE/dx vs E } \\
\mathrm{H}-\mathrm{Fe}, \mathrm{e}^{-} \\
\text {Energy Range } \\
2-40 \mathrm{MeV} / \text { nuc, species dependent (higher } \\
\text { energy not needed locally), } 6 \text { energy bins/decade }\end{array}$ \\
$\begin{array}{l}30^{\circ} \times 30^{\circ} \text { bins, all sky }(4 \pi) \\
\text { Resolution }\end{array}$ \\
$\begin{array}{l}\text { Time Resolution } \\
\text { GF \& SNR } \\
\begin{array}{l}\text { Mass Range \& } \\
\text { Resolution } \\
\text { Dynamic Range }\end{array}\end{array}$ & $\begin{array}{l}G F=4 \mathrm{~cm}^{2} \text { sr \& SNR }>100 \\
\left.\mathrm{M} / \Delta \mathrm{M} \geq 7 \text { (needed for }{ }^{3} \mathrm{He} /{ }^{4} \mathrm{He}\right)\end{array}$ \\
\hline
\end{tabular}

HIT (Fig. 20) is a standard $\mathrm{dE} / \mathrm{dx}$ versus $\mathrm{E}_{\text {tot }}$ spectrometer, where ionization losses, as a charged particle passes through multiple silicon SSDs, are used to characterize a particle's nuclear charge, energy, and mass. The instrument consists of a sensor head and electronics box, separated by a mechanical bracket. 
Fig. 20 Drawing of the HIT sensor head. HIT has ten L1 SSDs that define the instrument apertures. The particles stop in two L2 and two L3 SSDs at the center of the telescope

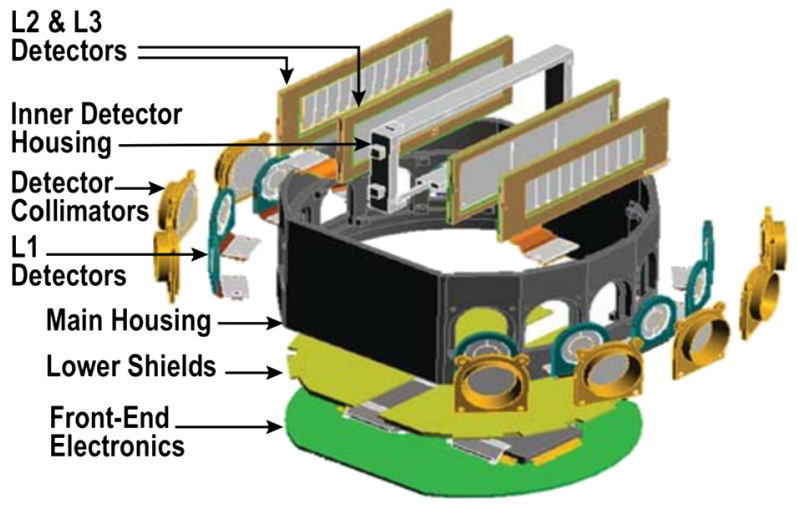

Fig. 21 STEREO/LET flight data showing a clear separation of all the major ion species in the SEPs

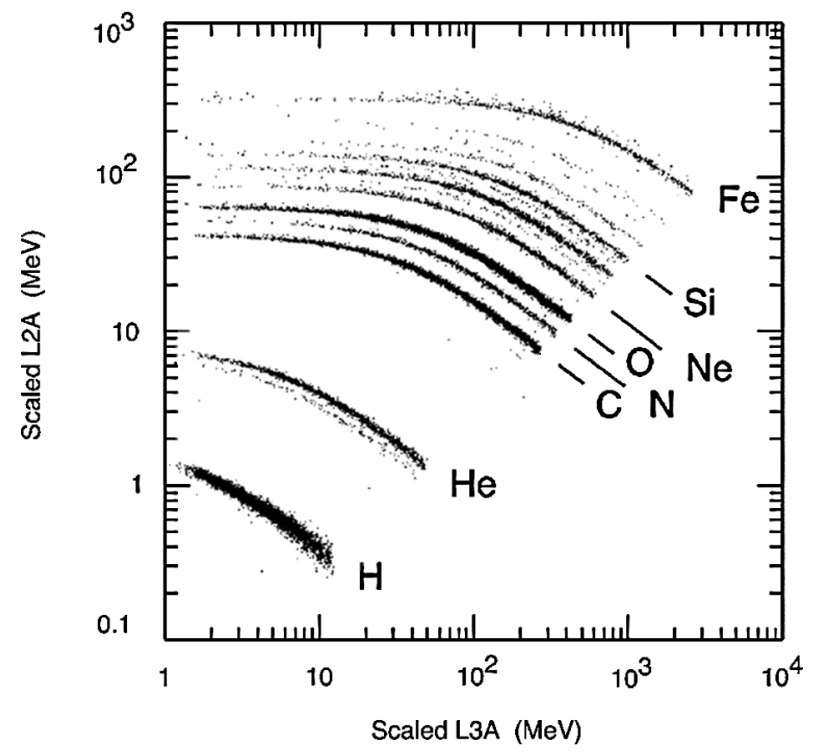

The L1 detectors have an active area of $2 \mathrm{~cm}^{2}$ split into three segments. Eight of the L1s are $24 \mu \mathrm{m}$ thick, as on STEREO/LET. These eight apertures, oriented perpendicular to the spin axis, sweep out a $4 \pi$, full-sky coverage. Two L1s, pointed in the sunward and anti-sunward directions are $1000 \mu \mathrm{m}$ thick, so that they are sensitive to electrons. These two apertures are used for the I-ALiRT space weather data stream, reporting electron fluxes from $\sim 0.5-1.0 \mathrm{MeV}$, which can be used as a predictor of SEP proton intensities that arrive later in an event.

The two L2 detectors are $50 \mu \mathrm{m}$ thick with an active area of $10.2 \mathrm{~cm}^{2}$ across 10 segments and the L3 detectors are $1000 \mu \mathrm{m}$ thick with an active area of $15.6 \mathrm{~cm}^{2}$ in two segments. The active area segmentation enables the measurement of arrival direction, which is used for angular path-length corrections and to observe SEP anisotropies. Figure 21 shows the ion resolution obtained with the LET instrument on STEREO-Ahead, demonstrating that the HIT design will meet the IMAP requirements. 
Table 13 IDEX instrument performance

Fig. 22 Cutaway diagram of the IDEX sensor head, showing the principle of operation

\begin{tabular}{ll}
\hline Parameter & Performance \\
\hline Instrument Type & Time-of-Flight Dust Spectrometer \\
FOV & $\pm 50^{\circ}$ \\
Mass Range, Res & $1-500 \mathrm{amu}, \mathrm{m} / \Delta \mathrm{m} \geq 200$ \\
\hline
\end{tabular}

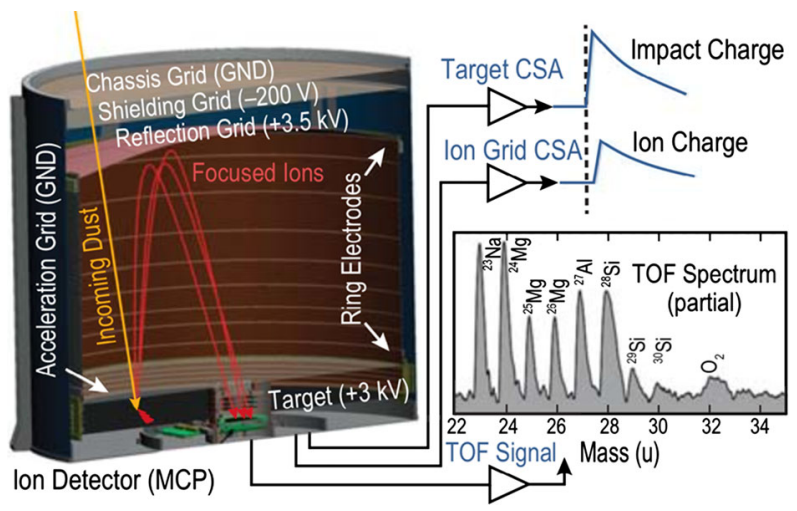

\subsection{IDEX}

The Interstellar Dust Experiment (IDEX) is a high-resolution dust analyzer that provides the elemental composition, speed and mass distributions of ISD particles (Altobelli et al. 2016). IDEX links the interstellar gas phase composition, as obtained with IMAP-Lo and through PUIs with CoDICE and SWAPI, with the makeup of ISD particles. IDEX's instrument performance parameters are summarized in Table 13.

IDEX has two components, an electronics enclosure and a sensor head with a large effective target area $\left(700 \mathrm{~cm}^{2}\right)$ to collect a statistically significant number of dust impacts (> 100/year), based on Ulysses-derived fluxes (Landgraf et al. 2003). The IDEX low-mass mechanical design consists of a simple Al shell structure that provides support for the biased electrodes of the TOF ion mass analyzer and the grids over its aperture. The centrally-located ion detector and the front-end charge sensitive amplifier are integrated into the bottom of the instrument.

A mass spectrum is obtained for each particle measured from the TOF analysis of the impact generated atomic and molecular ions (Srama et al. 2004; Sternovsky et al. 2007). Individual dust particles entering the instrument pass through a set of grid electrodes and impact the target (Fig. 22). The target is biased at $+3 \mathrm{kV}$ to provide positive ion acceleration and reflectron-type ion optics are used to generate TOF compositional mass spectra. An electrostatic field, shaped by a set of biased rings and a curved grid electrode, provides spatial and temporal focusing of the accelerated ions onto the central detector. IDEX's wide mass range of 1-500 amu is designed to identify the elemental makeup of impacting particles with selected mineralogical information. Organic molecules can be identified as well, if present.

Figure 22 also shows a mass spectrum from a prototype instrument and analog mineralogical dust particles obtained at a dust accelerator. The large ISD speeds increase the impact charge, that combined with IDEX geometry, provide a higher sensitivity than previous instruments, and an accurate dust mass estimate $(M / \Delta M>2)$ (Horanyi et al. 2014). 
Table 14 GLOWS instrument performance

\begin{tabular}{ll}
\hline Parameter & Performance \\
\hline Species & Hydrogen, Helium \\
Wavelengths & H: $121.6 \mathrm{~nm}$, He: $58.4 \mathrm{~nm}$; monochromatic \\
Angular Range \& Resolution & Almost-full-sky map per 6 mo $($ Ly- $\alpha) ; \pm 20^{\circ} \times 360^{\circ} ;$ Belt around ecliptic \\
& in He I $58.4 \mathrm{~nm}$ per 6 mo \\
Time Resolution & L $\alpha \mathrm{D}: 2 \mathrm{hr}$ for $4^{\circ}$ Belt, almost great circle Sun-centered; \\
& HeD: 2 hr for $4^{\circ}$ Belt, $40^{\circ}$ in diameter Sun-centered \\
SNR & L $\alpha \mathrm{D}:>100$ \\
& HeD: $>50$ after integration over a day \\
G-Factor & L $\alpha \mathrm{D}: 2$ counts/s/R \\
& HeD: 5 counts/s/R \\
Dynamic Range & H: max-to-min ratio $\sim 70$ \\
& He: max-to-min ratio up to 12 \\
& Dynamic range of instrument $>100$ \\
\end{tabular}

IDEX also enables excellent ion mass resolution $(\mathrm{m} / \Delta \mathrm{m}>200)$. The impact velocity of ISD particles is determined from orbital mechanics, combining IMAP motion around the Sun with ISD inflow. IDEX will be tested and calibrated at the dust accelerator of the University of Colorado (Shu et al. 2012). The interpretation of the measured ISD impact spectra is supported by continued laboratory calibration measurements using analog dust sample materials (Altobelli et al. 2016; Fiege et al. 2014; Hillier et al. 2012, 2018).

\subsection{GLOWS}

The GLObal solar Wind Structure (GLOWS) instrument measures the heliospheric resonant backscatter glow of hydrogen (the Lyman- $\alpha$ line at $121.6 \mathrm{~nm}$ ) and helium (at $58.4 \mathrm{~nm}$ ). GLOWS consists of two separate detectors: $\mathrm{L} \alpha \mathrm{D}$ and HeD for the two spectral channels, with the lines of sight directed at different angles with respect to the IMAP rotation axis, and a common on-board computer and power supply. The detectors are very similar in design, based on the successful LaD detector operating on the TWINS mission. The Lyman- $\alpha$ detector $\mathrm{L} \alpha \mathrm{D}$ is almost identical to LaD on TWINS, and HeD only has a new monochromator unit, which is a reflective grating behind a specially designed collimator baffle. GLOWS's instrument performance parameters are summarized in Table 14 and the basic scheme of the $\mathrm{HeD}$ detector is presented in Fig. 23.

GLOWS measures the modulation of the backscatter glow as a function of IMAP spin angle and its evolution during the Earth's travel around the Sun and with the changing solar activity (Fig. 24). The angular radii of the scanning circles of the two detectors are carefully selected so that the lines of sight intersect the solar polar axis in the geometric location where the respective maximum emissivity regions for the Lyman- $\alpha$ and He $58.4 \mathrm{~nm}$ glows are located. The distribution of the Lyman- $\alpha$ glow around the Sun reflects the latitudinal structure of the solar wind, existing because of the strong dependence of the charge-exchange ionization rate on the solar wind flux, which varies with heliolatitude (Bzowski et al. 2013b; Sokół et al. 2013). The distribution of the helium glow within $\sim 30^{\circ}$ from the Sun reflects ionization of ISN He by solar wind electron impact. With the solar wind structure known from Lyman- $\alpha$ measurements, observations of the modulation of the He $58.4 \mathrm{~nm}$ signal for the first time establish the temperature of 


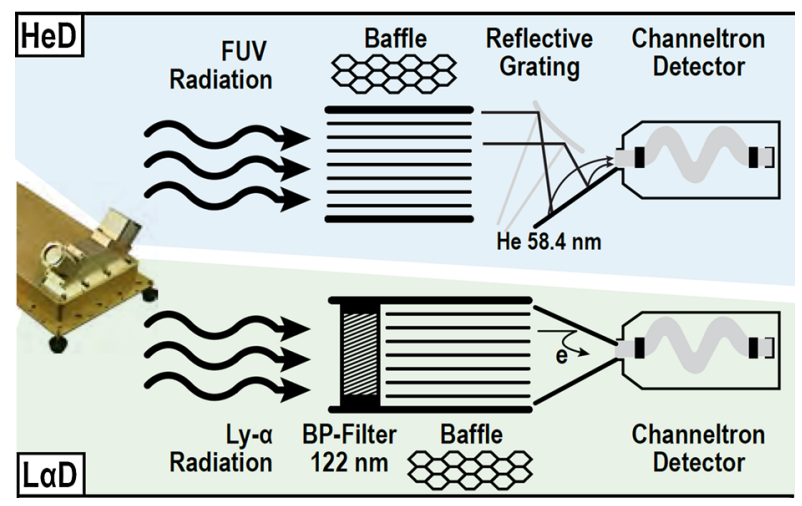

Fig. 23 Basic principles of the GLOWS HeD detector. The light enters the instrument through the baffle that defines the field of view and cuts off stray light. Inside, the light is spectrally split by the reflective grating and the $58.4 \mathrm{~nm}$ wavelength is directed to the channeltron detector. The pulses from the electrons emitted by the electrode due to the impinging extreme ultraviolet (EUV) photons are amplified and counted by the electronics. $\mathrm{L} \alpha \mathrm{D}$ is very similar but it features a filter in front of the baffle instead of the reflective grating behind the baffle

solar wind electrons close to the Sun at all heliolatitudes, which is fundamental to understanding the energy transport in the electron fluid near the Sun. In addition, observation of the helium glow, especially in March and September, establish the latitude of the flow direction of ISN helium because of the heliotail visibility (e.g., Vallerga et al. 2004; Lallement et al. 2004b), without interference from the Earth's exospheric glow.

The $\mathrm{L} \alpha \mathrm{D}$ and $\mathrm{HeD}$ detectors operate in synergy and provide a sufficient angular and time resolution to observe weekly variations of the solar wind structure and electron temperature. They also assist the determination of the interstellar He flow direction through the heliosphere. The extra-heliospheric (astrophysical) light sources will enable in-flight calibration and the inevitable sensitivity reduction tracing.

\section{Mission and Spacecraft}

IMAP operates in an L1 $10^{\circ} \times 5^{\circ}$ Lissajous orbit (similar to ACE). As on IBEX, the observatory is spin-stabilized at $4 \mathrm{rpm}$ with the spin axis biased on average several degrees to the right of the Sun, along the nominal incoming aberrated solar wind direction. This IMAP orbit, spin-rate, and pointing approach provide ideal conditions to perform both the required in situ and heliospheric interaction measurements. The primary in situ instruments (MAG, SWE, SWAPI, CoDICE, and HIT) measure the solar wind ions, PUIs, and EPs, continuously making measurements within the required two correlation lengths $(\sim 300,000 \mathrm{~km})$ from the Earth-Sun line throughout the orbit.

Heliospheric interaction instruments (IMAP-Lo, IMAP-Hi, IMAP-Ultra, IDEX, and GLOWS) also benefit from continuously operating at L1 and outside Earth's magnetosphere, significantly reducing measurement noise and foreground. ENA instruments (IMAP-Lo, -Hi, and -Ultra) are mounted to the observatory radially about the spin plane with complementing FOVs that are pointed at different angles away from the spin axis. These instruments as well as IDEX are shaded by the spacecraft. As the observatory completes a revolution, the ENA instruments measure a complete ring of the sky the width of their FOV. As the observatory repoints $\sim 1^{\circ} /$ day, the measurement rings fill the sky and complete a global 


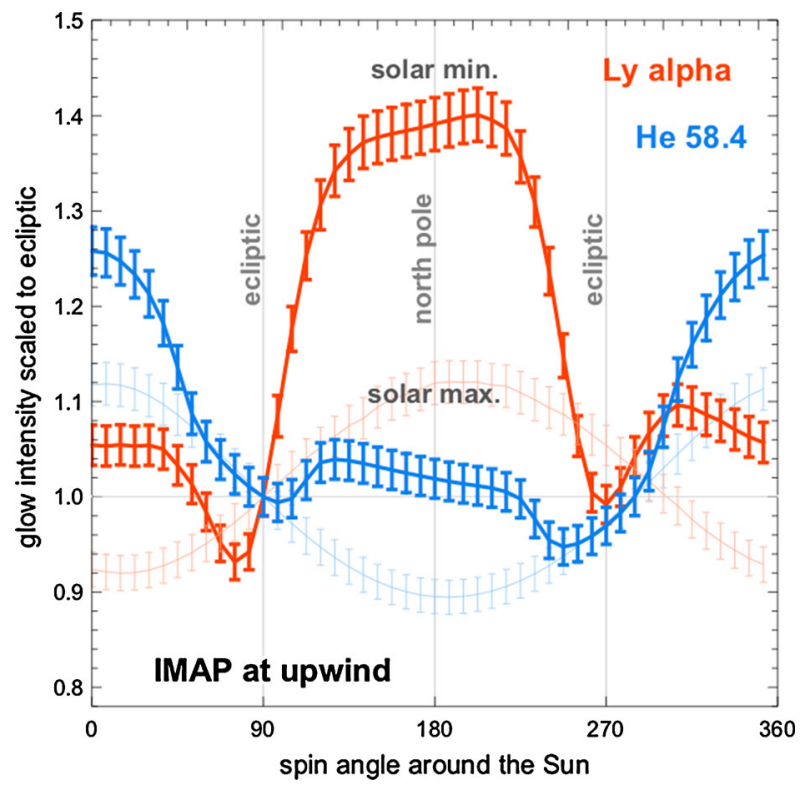

Fig. 24 Representative example of simulated measurements of the Lyman- $\alpha$ and He 58.4 nm backscatter glows, for IMAP sitting precisely at the upwind longitude. The intensities of the Ly- $\alpha$ (red) and He 58.4 (blue) glows, scaled to their respective values in the ecliptic plane, are shown for solar minimum (bold colors) and solar maximum conditions (pale colors). Error bars represent the expected magnitude of statistical scatter and background. The main source of the scatter for $\mathrm{L} \alpha \mathrm{D}$ are dim unresolved stars, for HeD the statistical scatter and penetrating radiation. Scanning of the glows in spin angle, with the spin axis pointing to the Sun, is equivalent to scanning them in heliolatitude. The signal modulation is due to (1) the small inclination of the ISN gas inflow direction to ecliptic (as seen for the solar maximum conditions, when solar wind is nearly spherically symmetric) and (2) due to the latitudinal structure of solar wind flux (Ly- $\alpha$ ) and the solar wind electron density and temperature (He 58.4), expected during solar minimum conditions. For observations from downwind, the plot will be mirror-reversed in spin angle. Measuring the latitude of the ISN gas inflow is best done using the He 58.4 glow from the crosswind position (not shown); the latitude is obtained from the spin angle of the maximum signal, which corresponds to the axis of the ISN He cone

map each six months, as on IBEX. Because IMAP-Hi and IMAP-Ultra have two sensors mounted at different angles, they cover more of the sky per revolution. IMAP-Lo adjusts its viewing direction with a pivot platform. The pivoting FOV allows us to simultaneously optimize IMAP-Lo for global heliospheric imaging and tracking the flow of ISN atoms. For IMAP-Lo, we plan to focus the first year on ENA viewing of the tail region $(10 \mathrm{eV}-1 \mathrm{keV})$, first global low-energy ENA maps $(0.1-1 \mathrm{keV})$, and the ISN He and O peak. In the second year, we plan to focus on the possible fine structure in ENAs $(0.1-1 \mathrm{keV})$ for portions of the Ribbon, and by following the flux peak of ISN D and Ne, provide the first accurate determinations of the $\mathrm{D} / \mathrm{H}$ and $\mathrm{Ne} / \mathrm{O}$ ratios.

Periodic, in-space cross-calibrations occur between IMAP-Lo, -Hi, and -Ultra using the overlapping energy ranges and sky coverage. Additional IMAP-Lo and IMAP-Hi in-flight cross-calibration is done with IBEX-Lo and IBEX-Hi during the 6 months after first data collection and continuously thereafter for as long as IBEX lasts. CoDICE-Lo and SWAPI and CoDICE-Hi and HIT are cross-calibrated routinely in flight. GLOWS is calibrated in flight by measuring EUV bright stars (standard candles). The baseline science phase of two years is sufficient to produce four six-month, full-sky maps from $100 \mathrm{eV}$ to $300 \mathrm{keV}$. 
Table 15 ACE-heritage real-time parameters at higher cadence

\begin{tabular}{llll}
\hline Product & $\begin{array}{l}\text { IMAP } \\
\text { Source }\end{array}$ & $\begin{array}{l}\text { ACE Real-Time } \\
\text { Cadence }\end{array}$ & $\begin{array}{l}\text { IMAP I-ALiRT } \\
\text { Cadence }\end{array}$ \\
\hline Mag Field & MAG & $60 \mathrm{~s}$ & $3 \mathrm{~s}$ \\
SW Thermal Plasma & SWAPI & $64 \mathrm{~s}$ & $15 \mathrm{~s}$ \\
Suprathermal Ions & CoDICE $_{H i}$ & $5 \mathrm{~min}$ & $1 \mathrm{~min}$ \\
Relativistic Protons & HIT & $5 \mathrm{~min}$ & $1 \mathrm{~min}$ \\
\hline
\end{tabular}

Spacecraft resources are provided to enable an extended mission to at least five years and likely much longer.

IMAP has two 4-hr telemetry contacts per week, which satisfies NAV requirements and meets commanding needs as well as downlinking all science and housekeeping data. IMAP also continuously broadcasts a small subset of the science data for I-ALiRT (500 bps) to supporting ground stations around the world when not in contact with the Deep Space Network (DSN). These stations transmit the data to the IMAP Science Operations Center (SOC) to process and disseminate to the space weather community.

The IMAP SOC at LASP is responsible for all aspects of instrument operations: planning, commanding, health and status monitoring, anomaly response, and sustaining engineering for the instruments. The SOC also handles science data processing (including data calibration, validation and preliminary analysis), distribution, archiving, and maintaining the IMAP data management plan. Science data are produced centrally using algorithms, software, and calibration data provided and managed by each instrument team. The SOC maintains a version-controlled but flexible, shareable software library, and higher-level science data products are produced in machine-independent self-describing formats (CDFs, FITS).

The NASA Space Physics Data Facility (SPDF) is the final archive for IMAP, with the SOC being responsible for archiving, and levels of data products are transferred to the SPDF regularly and made available through their Coordinated Data Analysis Web (CDAWeb) site with final versions archived at mission closeout. IDEX data are also archived in the Planetary Data System (PDS). The IMAP team shares all science and other data with the heliophysics community as rapidly as practical with an open data policy compliant with the NASA Heliophysics Science Data Management Policy.

\section{Real-Time Space Weather}

IMAP enhances critical real-time space weather data through its "IMAP Active Link for Real-Time" or I-ALiRT. Data include all of the important parameters currently provided by ACE, but at significantly higher cadence (Table 15), and also include several new key parameters. Continuation of the standard ACE data helps boost understanding of the science of space weather and makes progress in the understanding of the heliophysics system more quantifiable over time through forecasting metrics. Especially with the transition in 2016 of the Geospace Model from the University of Michigan's Space Weather Modeling Framework to NOAA, real-time data from L1 now drive operational, real-time space weather models directly. Even one bad solar wind density or velocity data point can result in errors, so IMAP's higher-cadence data will be useful for identifying and eliminating outliers and improving overall reliability of the predictions as well as providing more precise timing. 
Table 16 New space weather products available from IMAP

\begin{tabular}{lllr}
\hline Product & Description & Source & Cadence \\
\hline High-Energy Electron Fluxes & $\begin{array}{l}\sim 0.5-1.0 \mathrm{MeV}, \text { sunward and } \\
\text { anti-sunward } \\
\text { SW Charge-State Ratios }\end{array}$ & HIT & $1 \mathrm{~min}$ \\
& $\begin{array}{l}\mathrm{C}^{6+} / \mathrm{C}^{5+}, \mathrm{O}^{7+} / \mathrm{O}^{6+}, \mathrm{Mg} / \mathrm{O}, \mathrm{Fe} / \mathrm{O} \\
\mathrm{Fe}(\text { high Q }) / \mathrm{Fe}(\mathrm{low} \mathrm{Q})\end{array}$ & $\mathrm{CoDICE}_{L o}$ & $4 \mathrm{~min}$ \\
\hline
\end{tabular}

Equally important as extending the ACE real-time solar wind observations, is the testing of new space weather forecasting techniques driven by previously unavailable real-time data. Table 16 shows new space weather data from IMAP that may greatly enhance real-time forecasting. Enhanced electron fluxes associated with large solar events typically arrive well before the damaging protons and ions. Therefore, increases in electron flux can be used to predict the arrival time and intensity of solar energetic ions (Posner 2007). The solar wind $\mathrm{C}^{6+} / \mathrm{C}^{5+}, \mathrm{O}^{7+} / \mathrm{O}^{6+}, \mathrm{Mg} / \mathrm{O}, \mathrm{Fe} / \mathrm{O}$, and $\mathrm{Fe}$ charge-state ratios can also be used by forecasters for situational awareness. Together with other data, they allow identification of what types of solar wind are arriving at Earth: fast versus slow solar wind, ICME, CIR, or solar wind plasma sheet.

I-ALiRT follows the ACE, STEREO, and DSCOVR approach to provide continuous realtime products. Between DSN tracks, the flight system sends this low-rate stream to the SOC via a network of antenna partners located around the globe (e.g., Zwickl et al. 1998; Biesecker et al. 2008). During DSN tracks, the flight system includes the space weather data in the full-rate science data stream, which the Mission Operations Center (MOC) receives from the DSN and forwards to the SOC. In either case, the SOC processes this low-rate measurement data to create the space weather data products and posts them for users to retrieve. To provide data to forecasters in time for users to take protective action, I-ALiRT provides an end-to-end latency of no more than five minutes from the time of last measurement until the corresponding data product is available in the operational database.

\section{Heliophysics Community Engagement}

While the IMAP team already draws widely from across many of the leading Heliophysics institutions (Table 2), we seek even broader participation from the entire community. Thus, we included multiple opportunities for additional participation:

(1) We welcome outside scientists into the IMAP Science Team discussions. We are now holding these discussions jointly with the IBEX Science Team. Anyone interested in joining should contact the PI-Dave McComas (dmccomas at princeton.edu).

(2) We set aside $\$ 2 \mathrm{M}$ from our proposed cost cap for a Guest Investigator (GI) program. NASA will independently select IMAP GIs in Phase E; these GIs will participate as full IMAP team members, as the equivalent GIs did on IBEX.

(3) We set aside significant spacecraft resources for a TDO instrument for NASA to select separately. We plan to study the accommodation options and possibility of including a NASA-selected TDO on IMAP over Phase A.

(4) We have an inclusive Student Collaboration (Sect. 7.1).

(5) We are establishing and implementing an innovative Heliophysics Future Leaders (HFL) program (Sect. 7.2). 


\subsection{Student Collaboration}

The planned IMAP Student Collaboration augments the mission's science return, develops hands-on research experience for students, at least half of whom are women and/or members of underrepresented minorities, and contributes to diversifying space science. This program compliments the rest of the IMAP mission scientifically and provides students of diverse backgrounds with hands-on experience designing and building a CubeSat and two scientific instruments and with scientific involvement in studying the origin of EPs in the innermost magnetosphere.

Undergraduate, graduate, and high school students will design, develop, build, and launch a small satellite (a 6U CubeSat) with the science objective to determine how particles get injected into and accelerated in Earth's magnetosphere. This augments the fourth science objective of IMAP (O4) of "identifying and advancing understanding of particle injection and acceleration processes near the Sun, in the heliosphere, and in the heliosheath." The students will analyze the measurements of the CubeSat in combination with observations by IMAP and the rest of NASA's HSO to determine the relative importance of particles accelerated locally, particles accelerated in the corona and precipitating into the auroral regions, and particles accelerated at Earth's bow shock.

The IMAP Magnetospheric CubeSat (IMC) and instruments will be designed, developed, built, and operated by students at the IMAP student collaboration institutions, which include UNH, University of Colorado at Boulder (CU), Howard University (HU), a Historically Black College and University, and in partnership with chapters of the Society of Women Engineers at UNH and CU. Other participating institutions include APL, Morgan State University, and DC-area and female only high-schools. The inclusion of more partners will be pursued in Phase A to work on joint investigations with IMAP and IMC. The IMC carries two student-built instruments: (1) REPTile2, an EP detector (proton and electron) built at CU to detect injection and loss of EPs in Earth's magnetosphere and (2) an ESA built at UNH to determine the low-energy population of protons and positive ions in the inner magnetosphere.

\subsection{Heliophysics Future Leaders (HFL) Program}

To help address the ongoing lack of diversity in Heliophysics instrument and mission leadership, we look to the future and seek ways to develop and promote a far more diverse set of future leaders. IMAP science mentors pair with a set of diverse and high-achieving graduate students and postdocs throughout the IMAP mission development and science phases. HFL participants are chosen from a highly qualified pool of students and early career scientists identified both by team members and through solicitation of nearby colleges, especially those serving underrepresented groups.

Mentors help HFLs to understand mission design principles, requirements, and how to address relevant scientific topics in their particular areas of involvement. Five to ten future leaders comprise a peer cohort big enough to support each other in addition to the support given by the IMAP team. HFLs attend IMAP team meetings and are expected to present the work that they have been doing with their mentor. Specific skills that HFLs develop also include communication, both verbal and written, as well as time management, scientific team building, and networking.

Acknowledgements We are deeply indebted to all the other outstanding IMAP team members at all of our institutions that supported our proposal and continue to support development of this revolutionary mission. We also thank NASA and their review teams for their careful evaluation of our proposal in response to AO NNH17ZDA007O, as well as supporting the publication of this paper. 
Open Access This article is distributed under the terms of the Creative Commons Attribution 4.0 International License (http://creativecommons.org/licenses/by/4.0/), which permits unrestricted use, distribution, and reproduction in any medium, provided you give appropriate credit to the original author(s) and the source, provide a link to the Creative Commons license, and indicate if changes were made.

\section{References}

M.R. Aellig, H. Grünwaldt, P. Bochsler, S. Hefti, P. Wurz, R. Kallenbach, F.M. Ipavich, D. Hovestadt, M. Hilchenbach (the CELIAS Team), in Proc. 31st ESLAB Symposium, ESTEC, Noordwijk, The Netherlands. ESA, vol. SP-415 (1998a), pp. 27-31

M.R. Aellig, H. Grünwaldt, P. Bochsler, P. Wurz, S. Hefti, R. Kallenbach, W.I. Axford, H. Balsiger, A. Bürgi, M.A. Coplan, A.B. Galvin, J. Geiss, F. Gliem, G. Gloeckler, M. Hilchenbach, D. Hovestadt, K.C. Hsieh, F.M. Ipavich, B. Klecker, M.A. Lee, G.G. Managadze, E. Marsch, E. Möbius, M. Neugebauer, K.-U. Reiche, M. Scholer, M.I. Verigin, B. Wilken, J. Geophys. Res. 103, 17215 (1998b)

N. Altobelli, F. Postberg, K. Fiege, M. Trieloff, H. Kimura, V.J. Sterken, H.-W. Hsu, J. Hillier, N. Khawaja, G. Moragas-Klostermeyer, J. Blum, M. Burton, R. Srama, S. Kempf, E. Gruen, Science 352, 312 (2016)

F. Bagenal, P.A. Delamere, H.A. Elliott, M.E. Hill, C.M. Lisse, D.J. McComas, R.J. McNutt Jr., J.D. Richardson, C.W. Smith, D.F. Strobel, J. Geophys. Res. 120, 1497 (2015)

S.J. Bame, J.P. Glore, D.J. McComas, K.R. Moore, J.C. Chavez, T.J. Ellis, G.R. Peterson, J.H. Temple, F.J. Wymer, The ISPM Solar-Wind Plasma Experiment: European Space Agency Special Publication. ESA, vol. SP-1050 (European Space Agency, Paris, 1983), pp. 47-73

S.J. Bame, D.J. McComas, B.L. Barraclough, J.L. Phillips, K.J. Sofaly, J.C. Chavez, B.E. Goldstein, R.K. Sakurai, Astron. Astrophys. Suppl. Ser. 92, 237 (1992)

K. Bamert, R.F. Wimmer-Schweingruber, R. Kallenbach, M. Hilchenbach, B. Klecker, A. Bogdanov, P. Wurz, J. Geophys. Res. 107, 1130 (2002). https://doi.org/10.1029/2001JA900173

K. Bamert, R. Kallenbach, J.A. le Roux, M. Hilchenbach, C.W. Smith, P. Wurz, Astrophys. J. Lett. 675, L45 (2008)

B.L. Barraclough, E.E. Dors, R.A. Abeyta, J.F. Alexander, F.P. Ameduri, J.R. Baldonado, S.J. Bame, P.J. Casey, G. Dirks, D.T. Everett, J.T. Gosling, K.M. Grace, D.R. Guerrero, J.D. Kolar, J.L. Kroesche Jr., W.L. Lockhart, D.J. McComas, D.E. Mietz, J. Roese, J. Sanders, J.T. Steinberg, R.L. Tokar, C. Urdiales, R.C. Wiens, Space Sci. Rev. 105, 627 (2003)

J.L. Bertaux, J.E. Blamont, Astron. Astrophys. 11, 200 (1971)

D.A. Biesecker, D.F. Webb, O.C. St. Cyr, Space Sci. Rev. 136, 45 (2008)

P. Bochsler, L. Petersen, E. Möbius, N.A. Schwadron, P. Wurz, J.A. Scheer, S.A. Fuselier, D.J. McComas, M. Bzowski, P.C. Frisch, Astrophys. J. Suppl. Ser. 198, 13 (2012)

S.N. Borovikov, N.V. Pogorelov, Astrophys. J. Lett. 783, L16 (2014)

R. Bruno, V. Carbone, Turbulence in the Solar Wind (Springer, Berlin, 2016)

J.L. Burch, T.E. Moore, R.B. Torbert, B.L. Giles, Space Sci. Rev. 199, 5 (2016a)

J.L. Burch, R.B. Torbert, T.D. Phan, L.-J. Chen, T.E. Moore, R.E. Ergun, J.P. Eastwood, D.J. Gershman, P.A. Cassak, M.R. Argall, S. Wang, M. Hesse, C.J. Pollock, B.L. Giles, R. Nakamura, B.H. Mauk, S.A. Fuselier, C.T. Russell, R.J. Strangeway, J.F. Drake, M.A. Shay, Y.V. Khotyaintsev, P.-A. Lindqvist, G. Marklund, F.D. Wilder, D.T. Young, K. Torkar, J. Goldstein, J.C. Dorelli, L.A. Avanov, M. Oka, D.N. Baker, A.N. Jaynes, K.A. Goodrich, I.J. Cohen, D.L. Turner, J.F. Fennell, J.B. Blake, J. Clemmons, M. Goldman, D. Newman, S.M. Petrinec, K.J. Trattner, B. Lavraud, P.H. Reiff, W. Baumjohann, W. Magnes, M. Steller, W. Lewis, Y. Saito, V. Coffey, M. Chandler, Science 352, aaf2939 (2016b)

L.F. Burlaga, N.F. Ness, J.W. Belcher, Y.C. Whang, J. Geophys. Res. 101, 15523 (1996)

M. Bzowski, M.A. Kubiak, E. Moebius, P. Bochsler, T. Leonard, D. Heirtzler, H. Kucharek, J.M. Sokol, M. Hlond, G.B. Crew, N.A. Schwadron, S.A. Fuselier, D.J. McComas, Astrophys. J. Suppl. Ser. 198, 12 (2012)

M. Bzowski, J.M. Sokół, M.A. Kubiak, H. Kucharek, Astron. Astrophys. 557, A50 (2013a). https://doi.org/ 10.1051/0004-6361/201321700

M. Bzowski, J.M. Sokół, M. Tokumaru, K. Fujiki, E. Quémerais, R. Lallement, S. Ferron, P. Bochsler, D.J. McComas, in Cross-Calibration of Far UV Spectra of Solar System Objects and the Heliosphere, ed. by E. Quémerais, M. Snow, R.-M. Bonnet. ISSI Scientific Report Series, vol. 13 (Springer, New York, 2013b), p. 67

M. Bzowski, M.A. Kubiak, M. Hłond, J.M. Sokół, M. Banaszkiewicz, M. Witte, Astron. Astrophys. 569, A8 (2014)

M. Bzowski, P. Swaczyna, M.A. Kubiak, J.M. Sokół, S.A. Fuselier, A. Galli, D. Heirtzler, H. Kucharek, T.W. Leonard, D.J. McComas, E. Möbius, N.A. Schwadron, P. Wurz, Astrophys. J. Suppl. Ser. 220, 28 (2015) 
M. Bzowski, M.A. Kubiak, A. Czechowski, J. Grygorczuk, Astrophys. J. 845, 15 (2017)

S.V. Chalov, D.B. Alexashov, D. McComas, V.V. Izmodenov, Y.G. Malama, N. Schwadron, Astrophys. J. Lett. 716, L99 (2010)

A. Chasapis, W.H. Matthaeus, T.N. Parashar, S.A. Fuselier, B.A. Maruca, T.D. Phan, J.L. Burch, T.E. Moore, C.J. Pollock, D.J. Gershman, R.B. Torbert, C.T. Russell, R.J. Strangeway, Astrophys. J. Lett. 844, L9 (2017)

E. Chassefière, J.L. Bertaux, R. Lallement, V.G. Kurt, Astron. Astrophys. 160, 229 (1986)

M.A. Dayeh, M.I. Desai, J.R. Dwyer, H.K. Rassoul, G.M. Mason, J.E. Mazur, Astrophys. J. 693, 1588 (2009)

M.A. Dayeh, M.I. Desai, G.M. Mason, R.W. Ebert, A. Farahat, Astrophys. J. 835, 155 (2017)

M.I. Desai, G.M. Mason, J.E. Mazur, J.R. Dwyer, Astrophys. J. 649, 470 (2006)

M.I. Desai, F.A. Allegrini, M. Bzowski, M.A. Dayeh, H. Funsten, S.A. Fuselier, J. Heerikhuisen, M.A. Kubiak, D.J. McComas, N.V. Pogorelov, N.A. Schwadron, J.M. Sokół, G.P. Zank, E.J. Zirnstein, Astrophys. J. 780, 98 (2014)

M.I. Desai, K. Ogasawara, R.W. Ebert, D.J. McComas, F. Allegrini, S.E. Weidner, N. Alexander, S.A. Livi, Rev. Sci. Instrum. 856, 054501 (2015). https://doi.org/10.1063/1.4921706

M.I. Desai, M.A. Dayeh, F. Allegrini, D.J. McComas, H. Funsten, J. Heerikhuisen, S.A. Fuselier, N. Pogorelov, N.A. Schwadron, G.P. Zank, E.J. Zirnstein, P. Janzen, D.B. Reisenfeld, Astrophys. J. 832, 116 (2016a)

M.I. Desai, K. Ogasawara, R.W. Ebert, F. Allegrini, D.J. McComas, S. Livi, S.E. Weidner, J. Geophys. Res. 121, 6632 (2016b). https://doi.org/10.1002/2016JA022387

M.I. Desai, K. Ogasawara, D.J. McComas, S.A. Livi, Compact Dual Ion Composition Instrument. United States Patent No. 9,613,789 B1 (United States Patent and Trademark Office, Alexandria, VA, 2017)

K. Dialynas, S.M. Krimigis, D.G. Mitchell, R.B. Decker, E.C. Roelof, Nat. Astron. 1, 0115 (2017a)

K. Dialynas, S.M. Krimigis, D.G. Mitchell, R.B. Decker, E.C. Roelof, J. Phys. Conf. Ser. 900, 012005 (2017b). https://doi.org/10.1088/1742-6596/900/1/012005

J. Drake, M. Swisdak, M. Opher, Astrophys. J. Lett. 808, L44 (2015)

C. Drews, L. Berger, R.F. Wimmer-Schweingruber, P. Bochsler, A.B. Galvin, B. Klecker, E. Möbius, J. Geophys. Res. 117, A09106 (2012)

C.P. Escoubet, M.G.G.T. Taylor, A. Masson, H. Laakso, J. Volpp, M. Hapgood, M.L. Goldstein, Ann. Geophys. 31, 1045 (2013)

H.-J. Fahr, M. Siewert, D.J. McComas, N.A. Schwadron, Astron. Astrophys. 531, A77 (2011)

H. Fichtner, K. Scherer, F. Effenberger, J. Zönnchen, N. Schwadron, D.J. McComas, Astron. Astrophys. 561, A74 (2014)

K. Fiege, M. Trieloff, J.K. Hillier, M. Guglielmino, F. Postberg, R. Srama, S. Kempf, J. Blum, Icarus 241, 336 (2014)

L.A. Fisk, G. Gloeckler, Astrophys. J. 640, L79 (2006)

L.A. Fisk, G. Gloeckler, Space Sci. Rev. 173, 433 (2012)

L.A. Fisk, G. Gloeckler, J. Geophys. Res. 119, 1 (2014)

P.C. Frisch, Solar Journey: The Significance of Our Galactic Environment for the Heliosphere and Earth (Springer, Dordrecht, 2006)

P.C. Frisch, J.D. Slavin, Bull. Am. Astron. Soc. 34, 1281 (2002)

P.C. Frisch, S. Redfield, J.D. Slavin, Annu. Rev. Astron. Astrophys. 49, 237 (2011)

H.O. Funsten, D.J. McComas, B.L. Barraclough, Opt. Eng. 32, 3090 (1993)

H.O. Funsten, F. Allegrini, P. Bochsler, G. Dunn, S. Ellis, D. Everett, M.J. Fagan, S.A. Fuselier, M. Granoff, M. Gruntman, A.A. Guthrie, J. Hanley, R.W. Harper, D. Heirtzler, P. Janzen, K.H. Kihara, B. King, H. Kucharek, M.P. Manzo, M. Maple, K. Mashburn, D.J. McComas, E. Moebius, J. Nolin, D. Piazza, S. Pope, D.B. Reisenfeld, B. Rodriguez, E.C. Roelof, L. Saul, S. Turco, P. Valek, S. Weidner, P. Wurz, S. Zaffke, Space Sci. Rev. 146, 75 (2009a). https://doi.org/10.1007/s11214-009-9504-y

H.O. Funsten, F. Allegrini, G.B. Crew, R. DeMajistre, P.C. Frisch, S.A. Fuselier, M. Gruntman, P. Janzen, D.J. McComas, E. Möbius, B. Randol, D.B. Reisenfeld, E.C. Roelof, N.A. Schwadron, Science 326, 964 (2009b). https://doi.org/10.1126/science.1180927

H.O. Funsten, R.M. Skoug, A.A. Guthrie, E.A. MacDonald, J.R. Baldonado, R.W. Harper, K.C. Henderson, K.H. Kihara, J.E. Lake, B.A. Larsen, A.D. Puckett, V.J. Vigil, R.H. Friedel, M.G. Henderson, J.T. Niehof, G.D. Reeves, M.F. Thomsen, J.J. Hanley, D.E. George, J.-M. Jahn, S. Cortinas, A. De Los Santos, G. Dunn, E. Edlund, M. Ferris, M. Freeman, M. Maple, C. Nunez, T. Taylor, W. Toczynski, C. Urdiales, H.E. Spence, J.A. Cravens, L.L. Suther, J. Chen, Space Sci. Rev. 179, 423 (2013). https://doi.org/10.1007/s11214-013-9968-7

S.A. Fuselier, F. Allegrini, H.O. Funsten, A.G. Ghielmetti, D. Heirtzler, H. Kucharek, O.W. Lennartsson, D.J. McComas, E. Möbius, T.E. Moore, S.M. Petrinec, L.A. Saul, J.A. Scheer, N. Schwadron, P. Wurz, Science 326, 962 (2009a). https://doi.org/10.1126/science.1180981 
S.A. Fuselier, P. Bochsler, D. Chornay, G. Clark, G.B. Crew, G. Dunn, S. Ellis, T. Friedmann, H.O. Funsten, A.G. Ghielmetti, J. Googins, M.S. Granoff, J.W. Hamilton, J. Hanley, D. Heirtzler, E. Hertzberg, D. Isaac, B. King, U. Knauss, H. Kucharek, F. Kudirka, S. Livi, J. Lobell, S. Longworth, K. Mashburn, D.J. McComas, E. Möbius, A.S. Moore, T.E. Moore, R.J. Nemanich, J. Nolin, M. O’Neal, D. Piazza, L. Peterson, S.E. Pope, P. Rosmarynowski, L.A. Saul, J.R. Scherrer, J.A. Scheer, C. Schlemm, N.A. Schwadron, C. Tillier, S. Turco, J. Tyler, M. Vosbury, M. Wieser, P. Wurz, S. Zaffke, Space Sci. Rev. 146, 117 (2009b). https://doi.org/10.1007/s11214-009-9495-8

S.A. Fuselier, F. Allegrini, M. Bzowski, H.O. Funsten, A.G. Ghielmetti, G. Gloeckler, D. Heirtzler, P. Janzen, M. Kubiak, H. Kucharek, D.J. McComas, E. Möbius, T.E. Moore, S.M. Petrinec, M. Quinn, D. Reisenfeld, L.A. Saul, J.A. Scheer, N. Schwadron, K.J. Trattner, R. Vanderspek, P. Wurz, Astrophys. J. 754, 14 (2012)

K. Gamayunov, M. Zhang, H. Rassoul, Astrophys. J. 725, 2251 (2010)

K.V. Gamayunov, J. Heerikhuisen, H. Rassoul, Astrophys. J. 845, 63 (2017)

J. Giacalone, Astrophys. J. 609, 452 (2004)

J. Giacalone, Astrophys. J. 761, 28 (2012)

J. Giacalone, J.R. Jokipii, Astrophys. J. Lett. 812, L9 (2015)

J. Giacalone, D. Burgess, S.J. Schwartz, D.C. Ellison, Astrophys. J. 402, 550 (1993)

G. Gloeckler, L.A. Fisk, Astrophys. J. 806, L27 (2015)

G. Gloeckler, J. Geiss, Space Sci. Rev. 84, 275 (1998)

G. Gloeckler, J. Geiss, H. Balsiger, L.A. Fisk, A.B. Galvin, F.M. Ipavich, K.W. Ogilvie, R. von Steiger, B. Wilken, Science 261, 70 (1993)

G. Gloeckler, E. Möbius, J. Geiss, M. Bzowski, H. Noda, T. Terasawa, M. Oka, D. McMullin, S. Chalov, H. Fahr, D. Rucinski, R. von Steiger, A. Yamazaki, T. Zurbuchen, Astron. Astrophys. 426, 845 (2004)

J.T. Gosling, J.R. Asbridge, S.J. Bame, G. Paschmann, N. Sckopke, Geophys. Res. Lett. 5, 957 (1978)

S. Grzedzielski, M. Bzowski, A. Czechowski, H.O. Funsten, D.J. McComas, N.A. Schwadron, Astrophys. J. Lett. 715, L84 (2010)

J. Heerikhuisen, N.V. Pogorelov, G.P. Zank, G.B. Crew, P.C. Frisch, H.O. Funsten, P.H. Janzen, D.J. McComas, D.B. Reisenfeld, N.A. Schwadron, Astrophys. J. Lett. 708, L126 (2010)

J. Heerikhuisen, E.J. Zirnstein, H.O. Funsten, N.V. Pogorelov, G.P. Zank, Astrophys. J. 784, 73 (2014)

S. Hefti, H. Grünwaldt, F.M. Ipavich, P. Bochsler, D. Hovestadt, M.R. Aellig, M. Hilchenbach, R. Kallenbach, A.B. Galvin, J. Geiss, F. Gliem, G. Gloeckler, B. Klecker, E. Marsch, E. Möbius, M. Neugebauer, P. Wurz, J. Geophys. Res. 103, 29697 (1998)

J.K. Hillier, F. Postberg, S. Sestak, R. Srama, S. Kempf, M. Trieloff, Z. Sternovsky, S.F. Green, J. Geophys. Res. 117, E09002 (2012). https://doi.org/10.1029/2012JE004077

J.K. Hillier, Z. Sternovsky, S. Kempf, M. Trieloff, M. Guglielmino, F. Postberg, M.C. Price, Planet. Space Sci. 156, 96 (2018)

M. Hlond, M. Bzowski, E. Möbius, H. Kucharek, D. Heirtzler, N.A. Schwadron, M.E. O’Neill, G. Clark, G.B. Crew, S. Fuselier, D.J. McComas, Astrophys. J. Suppl. Ser. 198, 9 (2012). https://doi.org/10.1088/ 0067-0049/198/2/9

M. Horanyi, Z. Sternovsky, M. Lankton, C. Dumont, S. Gagnard, D. Gathright, E. Grün, D. Hansen, D. James, S. Kempf, B. Lamprecht, R. Srama, J.R. Szalay, G. Wright, Space Sci. Rev. 185, 93 (2014). https://doi.org/10.1007/s11214-014-0118-7

P.A. Isenberg, Astrophys. J. 787, 76 (2014)

E.B. Jenkins, Astrophys. J. 700, 1299 (2009)

A. Johlander, S.J. Schwartz, A. Vaivads, Yu.V. Khotyaintsev, I. Gingell, I.B. Peng, S. Markidis, P.-A. Lindqvist, R.E. Ergun, G.T. Marklund, F. Plaschke, W. Magnes, R.J. Strangeway, C.T. Russell, H. Wei, R.B. Torbert, W.R. Paterson, D.J. Gershman, J.C. Dorelli, L.A. Avanov, B. Lavraud, Y. Saito, B.L. Giles, C.J. Pollock, J.L. Burch, Phys. Rev. Lett. 117, 165101 (2016)

R. Kallenbach, A. Czechowski, M. Hilchenbach, P. Wurz, ISSI Sci. Rep. 5, 203 (2006)

O.V. Khabarova, G.P. Zank, Astrophys. J. 843, 4 (2017)

O.V. Khabarova, G.P. Zank, G. Li, O.E. Malandraki, J.A. le Roux, G.M. Webb, Astrophys. J. 827, 122 (2016)

O.V. Khabarova, G.P. Zank, O.E. Malandraki, G. Li, J.A. le Roux, G.M. Webb, Sun Geosph. 12, 23 (2017)

S.M. Krimigis, D.G. Mitchell, D.C. Hamilton, S. Livi, J. Dandouras, S. Jaskulek, T.P. Armstrong, J.D. Boldt, A.F. Cheng, G. Gloeckler, J.R. Hayes, K.C. Hsieh, W.-H. Ip, E.P. Keath, E. Kirsch, N. Krupp, L.J. Lanzerotti, R. Lundgren, B.H. Mauk, R.W. McEntire, E.C. Roelof, C.E. Schlemm, B.E. Tossman, B. Wilken, D.J. Williams, Space Sci. Rev. 114, 233 (2004)

S.M. Krimigis, D.G. Mitchell, E.C. Roelof, K.C. Hsieh, D.J. McComas, Science 326, 971 (2009)

M.A. Kubiak, M. Bzowski, J.M. Sokół, P. Swaczyna, S. Grzedzielski, D.B. Alexashov, V.V. Izmodenov, E. Möbius, T. Leonard, S.A. Fuselier, P. Wurz, D.J. McComas, Astrophys. J. Suppl. Ser. 213, 29 (2014)

M.A. Kubiak, P. Swaczyna, M. Bzowski, J.M. Sokół, S.A. Fuselier, A. Galli, D. Heirtzler, H. Kucharek, T.W. Leonard, D.J. McComas, E. Moebius, J. Park, N.A. Schwadron, P. Wurz, Astrophys. J. Suppl. Ser. 223, 25 (2016) 
H. Kucharek, S.A. Fuselier, P. Wurz, N. Pogorelov, S. Borovikov, M.A. Lee, E. Moebius, D. Reisenfeld, H. Funsten, N. Schwadron, D. McComas, Astrophys. J. 776, 109 (2013)

R. Kumar, E.J. Zirnstein, A. Spitkovsky, Astrophys. J. 860, 156 (2018)

R. Lallement, J.C. Raymond, J.-L. Bertaux, E. Quémerais, Y.-K. Ko, M. Uzzo, D. McMullin, D. Rucinski, Astron. Astrophys. 426, 867 (2004a)

R. Lallement, J.C. Raymond, J. Vallerga, M. Lemoine, F. Dalaudier, J.L. Bertaux, Astron. Astrophys. 426, $875(2004 b)$

R. Lallement, E. Quémerais, J.L. Bertaux, S. Ferron, D. Koutroumpa, R. Pellinen, Science 307, 1447 (2005)

M. Landgraf, H. Krüger, N. Altobelli, E. Grün, J. Geophys. Res. 108, 8030 (2003). https://doi.org/10.1029/ 2003JA009872

J.L. Linsky, Space Sci. Rev. 106, 49 (2003)

J.L. Linsky, Space Sci. Rev. 130, 367 (2007)

J.L. Linsky, B.T. Draine, H.W. Moos, E.B. Jenkins, B.E. Wood, C. Oliveira, W.P. Blair, S.D. Friedman, C. Gry, D. Knauth, J.W. Kruk, S. Lacour, N. Lehner, S. Redfield, J.M. Shull, G. Sonneborn, G.M. Williger, Astrophys. J. 647, 1106 (2006)

G. Livadiotis, D.J. McComas, J. Geophys. Res. 114, A11105 (2009)

G. Livadiotis, D.J. McComas, Space Sci. Rev. 175, 183 (2013)

W.H. Matthaeus, M. Velli, Space Sci. Rev. 160, 145 (2011)

B.H. Mauk, J.B. Blake, D.N. Baker, J.H. Clemmons, G.D. Reeves, H.E. Spence, S.E. Jaskulek, C.E. Schlemm, L.E. Brown, S.A. Cooper, J.V. Craft, J.F. Fennell, R.S. Gurnee, C.M. Hammock, J.R. Hayes, P.A. Hill, G.C. Ho, J.C. Hutcheson, A.D. Jacques, S. Kerem, D.G. Mitchell, K.S. Nelson, N.P. Paschalidis, E. Rossano, M.R. Stokes, J.H. Westlake, Space Sci. Rev. 199, 471 (2016)

C. Mazelle, K. Meziane, D. Le Quéau, M. Wilber, J.P. Eastwood, H. Rème, J.A. Sauvaud, J.M. Bosqued, I. Dandouras, M. McCarthy, L.M. Kistler, B. Klecker, A. Korth, M.B. Bavassano-Cattaneo, G. Pallocchia, R. Lundin, A. Balogh, Planet. Space Sci. 51, 785 (2003)

D.J. McComas, S.J. Bame, P. Barker, W.C. Feldman, J.L. Phillips, P. Riley, J.W. Griffee, Space Sci. Rev. 86, 563 (1998)

D.J. McComas, F. Allegrini, C.J. Pollock, H.O. Funsten, S. Ritzau, G. Gloeckler, Rev. Sci. Instrum. 75, 4863 (2004)

D. McComas, F. Allegrini, F. Bagenal, P. Casey, P. Delamere, D. Demkee, G. Dunn, H. Elliott, J. Hanley, K. Johnson, J. Langle, G. Miller, S. Pope, M. Reno, B. Rodriguez, N. Schwadron, P. Valek, S. Weidner, Space Sci. Rev. 140, 261 (2008)

D.J. McComas, F. Allegrini, P. Bochsler, M. Bzowski, M. Collier, H. Fahr, H. Fichtner, P. Frisch, H.O. Funsten, S.A. Fuselier, G. Gloeckler, M. Gruntman, V. Izmodenov, P. Knappenberger, M. Lee, S. Livi, D. Mitchell, E. Möbius, T. Moore, S. Pope, D. Reisenfeld, E. Roelof, J. Scherrer, N. Schwadron, R. Tyler, M. Wieser, M. Witte, P. Wurz, G. Zank, Space Sci. Rev. 146, 11 (2009a)

D.J. McComas, F. Allegrini, P. Bochsler, M. Bzowski, E.R. Christian, G.B. Crew, R. DeMajistre, H. Fahr, H. Fichtner, P.C. Frisch, H.O. Funsten, S.A. Fuselier, G. Gloeckler, M. Gruntman, J. Heerikhuisen, V. Izmodenov, P. Janzen, P. Knappenberger, S. Krimigis, H. Kucharek, M. Lee, G. Livadiotis, S. Livi, R.J. MacDowall, D. Mitchell, E. Möbius, T. Moore, N.V. Pogorelov, D. Reisenfeld, E. Roelof, L. Saul, N.A. Schwadron, P.W. Valek, R. Vanderspek, P. Wurz, G.P. Zank, Science 326, 959 (2009b)

D.J. McComas, D. Alexashov, M. Bzowski, H. Fahr, J. Heerikhuisen, V. Izmodenov, M.A. Lee, E. Möbius, N. Pogorelov, N.A. Schwadron, G.P. Zank, Science 336, 1291 (2012)

D.J. McComas, M.A. Dayeh, H.O. Funsten, G. Livadiotis, N.A. Schwadron, Astrophys. J. 771, 77 (2013)

D.J. McComas, W.S. Lewis, N.A. Schwadron, Rev. Geophys. 52, 118 (2014)

D.J. McComas, M. Bzowski, S.A. Fuselier, P.C. Frisch, A. Galli, V.V. Izmodenov, O.A. Katushkina, M.A. Kubiak, M.A. Lee, T.W. Leonard, E. Möbius, J. Park, N.A. Schwadron, J.M. Sokół, P. Swaczyna, B.E. Wood, P. Wurz, Astrophys. J. Suppl. Ser. 220, 22 (2015a)

D.J. McComas, M. Bzowski, P. Frisch, S.A. Fuselier, M.A. Kubiak, H. Kucharek, T. Leonard, E. Möbius, N.A. Schwadron, J.M. Sokół, P. Swaczyna, M. Witte, Astrophys. J. 801, 28 (2015b)

D.J. McComas, E.J. Zirnstein, M. Bzowski, M.A. Dayeh, H.O. Funsten, S.A. Fuselier, P.H. Janzen, M.A. Kubiak, H. Kucharek, E. Möbius, D.B. Reisenfeld, N.A. Schwadron, J.M. Sokół, J.R. Szalay, M. Tokumaru, Astrophys. J. Suppl. Ser. 229, 41 (2017a)

D.J. McComas, E.J. Zirnstein, M. Bzowski, H.A. Elliott, B. Randol, N.A. Schwadron, J.M. Sokól, J.R. Szalay, C. Olkin, J. Spencer, A. Stern, H. Weaver, Astrophys. J. Suppl. Ser. 233, 8 (2017b)

D.J. McComas, M.A. Dayeh, H.O. Funsten, J. Heerikhuisen, P.H. Janzen, D.B. Reisenfeld, N.A. Schwadron, J.R. Szalay, E.J. Zirnstein, Astrophys. J. Lett. 856, L10 (2018)

R.A. Mewaldt, G.M. Mason, G. Gloeckler, E.R. Christian, C.M.S. Cohen, A.C. Cummings, A.J. Davis, J.R. Dwyer, R.E. Gold, S.M. Krimigis, R.A. Leske, J.E. Mazur, E.C. Stone, T.T. von Rosenvinge, M.E. Wiedenbeck, T.H. Zurbuchen, AIP Conf. Proc. 598, 165 (2001). https://doi.org/10.1063/1.1433995 
R.A. Mewaldt, C.M.S. Cohen, W.R. Cook, A.C. Cummings, A.J. Davis, S. Geier, B. Kecman, J. Klemic, A.W. Labrador, R.A. Leske, H. Miyasaka, V. Nguyen, R.C. Ogliore, E.C. Stone, R.G. Radocinski, M.E. Wiedenbeck, J. Hawk, S. Shuman, T.T. von Rosenvinge, K. Wortman, Space Sci. Rev. 136, 285 (2008)

K. Meziane, M. Wilber, A.M. Hamza, C. Mazelle, G.K. Parks, H. Rème, E.A. Lucek, J. Geophys. Res. Space Phys. 112, 2156 (2007)

A.H. Minter, S.R. Spangler, Astrophys. J. 458, 194 (1996)

D.G. Mitchell, P.C. Brandt, J.H. Westlake, S.E. Jaskulek, G.B. Andrews, K.S. Nelson, J. Geophys. Res. 121, 8804 (2016). https://doi.org/10.1002/2016JA022586

E. Möbius, D. Hovestadt, B. Klecker, M. Scholer, G. Gloeckler, F.M. Ipavich, Nature 318, 426 (1985)

E. Möbius, B. Klecker, D. Hovestadt, M. Scholer, Astrophys. Space Sci. 144, 487 (1988)

E. Möbius, S. Fuselier, M. Granoff, E. Hertzberg, B. King, H. Kucharek, S. Livi, S. Longworth, N. Paschalidis, L. Saul, J. Scheer, C. Schlemm, M. Wieser, P. Wurz, in Proc. of the 30th Int. Cosmic Ray Conf., vol. 1, Universidad Nacional Autónoma de México, Mexico City, Mexico (2008), pp. 841-844

E. Möbius, P. Bochsler, M. Bzowski, G.B. Crew, H.O. Funsten, S.A. Fuselier, A. Ghielmetti, D. Heirtzler, V.V. Izmodenov, M. Kubiak, H. Kucharek, M.A. Lee, T. Leonard, D.J. McComas, L. Petersen, L. Saul, J.A. Scheer, N. Schwadron, M. Witte, P. Wurz, Science 326, 969 (2009)

E. Möbius, P. Bochsler, M. Bzowski, D. Heirtzler, M.A. Kubiak, H. Kucharek, M.A. Lee, T. Leonard, N.A. Schwadron, X. Wu, S.A. Fuselier, G. Crew, D.J. McComas, L. Petersen, L. Saul, D. Valovcin, R. Vanderspek, P. Wurz, Astrophys. J. Suppl. Ser. 198, 11 (2012)

E. Möbius, M. Bzowski, P.C. Frisch, S.A. Fuselier, D. Heirtzler, M.A. Kubiak, H. Kucharek, M.A. Lee, T. Leonard, D.J. McComas, N.A. Schwadron, J.M. Sokół, P. Swaczyna, P. Wurz, Astrophys. J. Suppl. Ser. 220, 24 (2015a)

E. Möbius, M.A. Lee, C. Drews, Astrophys. J. 815, 20 (2015b)

National Research Council, Division on Engineering and Physical Sciences, Space Studies Board, Aeronautics and Space Engineering Board, Committee on a Decadal Strategy for Solar and Space Physics (Heliophysics), Science for a Technological Society: The 2013-2022 Decadal Survey in Solar and Space Physics (National Academies Press, Washington, DC, 2013). https://doi.org/10.17226/13060

K. Ogasawara, S.A. Livi, F. Allegrini, T.W. Broiles, M.A. Dayeh, M.I. Desai, R.W. Ebert, K. Llera, S.K. Vines, D.J. McComas, J. Geophys. Res. 121, 6075 (2016). https://doi.org/10.1002/2016JA022559

M. Opher, J.F. Drake, B. Zieger, T.I. Gombosi, Astrophys. J. Lett. 800, L28 (2015)

J. Park, H. Kucharek, E. Möbius, T. Leonard, M. Bzowski, J.M. Sokół, M.A. Kubiak, S.A. Fuselier, D.J. McComas, Astrophys. J. 795, 97 (2014)

J. Park, H. Kucharek, E. Möbius, A. Galli, M.A. Kubiak, M. Bzowski, D.J. McComas, Astrophys. J. 833, 130 (2016)

E.N. Parker, Astrophys. J. 128, 664 (1958)

E.N. Parker, Astrophys. J. 134, 20 (1961)

N.V. Pogorelov, S.N. Borovikov, J. Heerikhuisen, M. Zhang, Astrophys. J. Lett. 812, L6 (2015)

N.V. Pogorelov, H. Fichtner, A. Czechowski, A. Lazarian, B. Lembege, J.A. Le Roux, M.S. Potgieter, K. Scherer, E.C. Stone, R.D. Strauss, T. Wiengarten, P. Wurz, G.P. Zank, M. Zhang, Space Sci. Rev. 212, 193 (2017a)

N.V. Pogorelov, J. Heerikhuisen, V. Roytershteyn, L.F. Burlaga, D.A. Gurnett, W.S. Kurth, Astrophys. J. 845, 9 (2017b)

A. Posner, Space Weather 5, S05001 (2007). https://doi.org/10.1029/2006SW000268

R.A. Rhode, R.A. Muller, Nature 434, 208 (2005). https://doi.org/10.1038/nature03339

D.F. Rodríguez Moreno, P. Wurz, L. Saul, M. Bzowski, M.A. Kubiak, J.M. Sokół, P. Frisch, S.A. Fuselier, D.J. McComas, E. Möbius, N. Schwadron, Astron. Astrophys. 557, A125 (2013)

D. Rodríguez Moreno, P. Wurz, L. Saul, M. Bzowski, M. Kubiak, J. Sokół, P. Frisch, S. Fuselier, D. McComas, E. Möbius, N. Schwadron, Entropy 16, 1134 (2014)

D. Ruciński, H.J. Fahr, Ann. Geophys. 9, 102 (1991)

C.T. Russell, M.M. Hoppe, W.A. Livesey, J.T. Gosling, S.J. Bame, Geophys. Res. Lett. 9, 1171 (1982)

C.T. Russell, H.K. Leinweber, R.A. Hart, H.Y. Wei, R.J. Strangeway, T.L. Zhang, Icarus 226, 1527 (2013a). https://doi.org/10.1016/j.icarus.2013.08.019

C.T. Russell, R.A. Mewaldt, J.G. Luhmann, G.M. Mason, T.T. von Rosenvinge, C.M.S. Cohen, R.A. Leske, R. Gomez-Herrero, A. Klassen, A.B. Galvin, K.D.C. Simunac, Astrophys. J. 770, 38 (2013b). https://doi.org/10.1088/0004-637x/770/1/38

C.T. Russell, B.J. Anderson, W. Baumjohann, K.R. Bromund, D. Dearborn, D. Fischer, G. Le, H. Leinweber, D. Leneman, W. Magnes, J.D. Means, M.B. Moldwin, R. Nakamura, D. Pierce, F. Plaschke, K.M. Rowe, J.A. Slavin, R.J. Strangeway, R. Torbert, C. Hagen, I. Jernej, A. Valavanoglou, I. Richter, Space Sci. Rev. 199, 189 (2016). https://doi.org/10.1007/s11214-014-0057-3

J.A. Scheer, M. Wieser, P. Wurz, P. Bochsler, E. Hertzberg, S.A. Fuselier, F.A. Koeck, R.J. Nemanich, M. Schleberger, Nucl. Instrum. Methods Phys. Res., Sect. B 230, 330 (2005). https://doi.org/10.1016/ j.nimb.2004.12.063 
J.A. Scheer, M. Wieser, P. Wurz, P. Bochsler, E. Hertzberg, S.A. Fuselier, F.A. Koeck, R.J. Nemanich, M. Schleberger, Adv. Space Res. 38, 664 (2006)

K. Scherer, H. Fichtner, O. Stawicki, J. Atmos. Sol.-Terr. Phys. 64, 795 (2002). https://doi.org/10.1016/ S1364-6826(02)00078-0

M. Scholer, M.F. Thomsen, D. Burgess, S.D. Bale, M.A. Balikhin, A. Balogh, T.S. Horbury, V.V. Krasnoselskikh, H. Kucharek, E.A. Lucek, B. Lembège, E. Möbius, S.J. Schwartz, S.N. Walker, Space Sci. Rev. 118, 223 (2005)

N.A. Schwadron, M. Bzowski, Astrophys. J. 862, 11 (2018)

N.A. Schwadron, D.J. McComas, Astrophys. J. 764, 92 (2013)

N.A. Schwadron, L.A. Fisk, G. Gloeckler, Geophys. Res. Lett. 23, 2871 (1996)

N.A. Schwadron, M. Bzowski, G.B. Crew, M. Gruntman, H. Fahr, H. Fichtner, P.C. Frisch, H.O. Funsten, S. Fuselier, J. Heerikhuisen, V. Izmodenov, H. Kucharek, M. Lee, G. Livadiotis, D.J. McComas, E. Moebius, T. Moore, J. Mukherjee, N.V. Pogorelov, C. Prested, D. Reisenfeld, E. Roelof, G.P. Zank, Science 326, 966 (2009)

N.A. Schwadron, L.A. Fisk, G. Gloeckler, Astrophys. J. 713, 1386 (2010)

N.A. Schwadron, H.E. Spence, R. Came, Eos 92, 297 (2011)

N.A. Schwadron, E. Moebius, H. Kucharek, M.A. Lee, J. French, L. Saul, P. Wurz, M. Bzowski, S.A. Fuselier, G. Livadiotis, D.J. McComas, P. Frisch, M. Gruntman, H.R. Mueller, Astrophys. J. 775, 86 (2013)

N.A. Schwadron, J.B. Blake, A.W. Case, C.J. Joyce, J. Kasper, J. Mazur, N. Petro, M. Quinn, J.A. Porter, C.W. Smith, S. Smith, H.E. Spence, L.W. Townsend, R. Turner, J.K. Wilson, C. Zeitlin, Space Weather 12, 622 (2014a)

N.A. Schwadron, E. Moebius, S.A. Fuselier, D.J. McComas, H.O. Funsten, P. Janzen, D. Reisenfeld, H. Kucharek, M.A. Lee, K. Fairchild, F. Allegrini, M. Dayeh, G. Livadiotis, M. Reno, M. Bzowski, J.M. Sokół, M.A. Kubiak, E.R. Christian, R. DeMajistre, P. Frisch, A. Galli, P. Wurz, M. Gruntman, Astrophys. J. Suppl. Ser. 215, 13 (2014b)

N.A. Schwadron, E. Möbius, T. Leonard, S.A. Fuselier, D.J. McComas, D. Heirtzler, H. Kucharek, F. Rahmanifard, M. Bzowski, M.A. Kubiak, J.M. Sokół, P. Swaczyna, P. Frisch, Astrophys. J. Suppl. Ser. 220, 25 (2015)

N.A. Schwadron, E. Möbius, D.J. McComas, P. Bochsler, M. Bzowski, S.A. Fuselier, G. Livadiotis, P. Frisch, H.-R. Müller, D. Heirtzler, H. Kucharek, M.A. Lee, Astrophys. J. 828, 81 (2016a)

N.A. Schwadron, M. Opher, J. Kasper, R. Mewaldt, E. Moebius, H.E. Spence, T.H. Zurbuchen, J. Phys. Conf. Ser. 767, 012025 (2016b)

N.J. Shaviv, J. Veizer, GSA Today 13, 4 (2003). https://doi.org/10.1130/1052-5173(2003)013<0004: CDOPC $>2.0 . \mathrm{CO} ; 2$

A. Shu, A. Collette, K. Drake, E. Grün, M. Horányi, S. Kempf, A. Mocker, T. Munsat, P. Northway, R. Srama, Z. Sternovsky, E. Thomas, Rev. Sci. Instrum. 83, 075108 (2012)

M. Siewert, H.-J. Fahr, D.J. McComas, N.A. Schwadron, Astron. Astrophys. 539, A75 (2012)

M. Siewert, H.-J. Fahr, D.J. McComas, N.A. Schwadron, Astron. Astrophys. 551, A58 (2013)

J.D. Slavin, P.C. Frisch, Astron. Astrophys. 491, 53 (2008)

J.M. Sokół, M. Bzowski, M. Tokumaru, K. Fujiki, D.J. McComas, Sol. Phys. 285, 167 (2013). https://doi.org/ 10.1007/s11207-012-9993-9

J.M. Sokół, M. Bzowski, M.A. Kubiak, P. Swaczyna, A. Galli, P. Wurz, E. Möbius, H. Kucharek, S.A. Fuselier, D.J. McComas, Astrophys. J. Suppl. Ser. 220, 29 (2015a)

J.M. Sokół, M.A. Kubiak, M. Bzowski, P. Swaczyna, Astrophys. J. Suppl. Ser. 220, 27 (2015b). https:// doi.org/10.1088/0067-0049/220/2/27

J.M. Sokół, M. Bzowski, M.A. Kubiak, E. Möbius, Mon. Not. R. Astron. Soc. 458, 3691 (2016)

R. Srama, T.J. Ahrens, N. Altobelli, S. Auer, J.G. Bradley, M. Burton, V.V. Dikarev, T. Economou, H. Fechtig, M. Gorlich, M. Grande, A. Graps, E. Grün, O. Havnes, S. Helfert, M. Horanyi, E. Igenbergs, E.K. Jessberger, T.V. Johnson, S. Kempf, A.V. Krivov, H. Krüger, A. Mocker-Ahlreep, G. MoragasKlostermeyer, P. Lamy, M. Landgraf, D. Linkert, G. Linkert, F. Lura, J.A.M. McDonnell, D. Mohlmann, G.E. Morfill, M. Muller, M. Roy, G. Schafer, G. Schlotzhauer, G.H. Schwehm, F. Spahn, M. Stubig, J. Svestka, V. Tschernjawski, A.J. Tuzzolino, R. Wasch, H.A. Zook, Space Sci. Rev. 114, 465 (2004). https://doi.org/10.1007/s11214-004-1435-Z

Z. Sternovsky, K. Amyx, G. Bano, M. Landgraf, M. Horányi, S. Knappmiller, S. Robertson, E. Grün, R. Srama, S. Auer, Rev. Sci. Instrum. 78, 014501 (2007)

P. Swaczyna, M. Bzowski, Astrophys. J. 846, 128 (2017)

P. Swaczyna, M. Bzowski, M.A. Kubiak, J.M. Sokół, S.A. Fuselier, D. Heirtzler, H. Kucharek, T.W. Leonard, D.J. McComas, E. Möbius, N.A. Schwadron, Astrophys. J. Suppl. Ser. 220, 26 (2015)

P. Swaczyna, S. Grzedzielski, M. Bzowski, Astrophys. J. 840, 75 (2017)

J.A. Tessein, W.H. Matthaeus, M. Wan, K.T. Osman, D. Ruffolo, J. Giacalone, Astrophys. J. Lett. 776, L8 (2013) 
G.E. Thomas, R.F. Krassa, Astron. Astrophys. 11, 218 (1971)

P. Todd, Adv. Space Res. 14, 305 (1994). https://doi.org/10.1016/0273-1177(94)90483-9

J. Vallerga, R. Lallement, J. Raymond, M. Lemoine, B. Flynn, F. Dalaudier, D. McMullin, Astron. Astrophys. 426, 855 (2004)

C.S. Weller, R.R. Meier, Astrophys. J. 193, 471 (1974)

A.J. Westphal, R.M. Stroud, H.A. Bechtel, F.E. Brenker, A.L. Butterworth, G.J. Flynn, D.R. Frank, Z. Gainsforth, J.K. Hillier, F. Postberg, A.S. Simionovici, V.J. Sterken, L.R. Nittler, C. Allen, D. Anderson, A. Ansari, S. Bajt, R.K. Bastien, N. Bassim, J. Bridges, D.E. Brownlee, M. Burchell, M. Burghammer, H. Changela, P. Cloetens, A.M. Davis, R. Doll, C. Floss, E. Grün, P.R. Heck, P. Hoppe, B. Hudson, J. Huth, A. Kearsley, A.J. King, B. Lai, J. Leitner, L. Lemelle, A. Leonard, H. Leroux, R. Lettieri, W. Marchant, R. Ogliore, W.J. Ong, M.C. Price, S.A. Sandford, J.-A.S. Tresseras, S. Schmitz, T. Schoonjans, K. Schreiber, G. Silversmit, V.A. Solé, R. Srama, F. Stadermann, T. Stephan, J. Stodolna, S. Sutton, M. Trieloff, P. Tsou, T. Tyliszczak, B. Vekemans, L. Vincze, J. Von Korff, N. Wordsworth, D. Zevin, M.E. Zolensky, Science 345, 786 (2014)

M. Wieser, P. Wurz, E. Möbius, S.A. Fuselier, E. Hertzberg, D.J. McComas, Rev. Sci. Instrum. 78, 124502-1 (2007)

M. Witte, Astron. Astrophys. 426, 835 (2004)

B.E. Wood, H.-R. Müller, M. Bzowski, J.M. Sokół, E. Möbius, M. Witte, D.J. McComas, Astrophys. J. Suppl. Ser. 220, 31 (2015)

P. Wurz, R. Schletti, M.R. Aellig, Surf. Sci. 373, 56 (1997)

P. Wurz, J. Scheer, M. Wieser, E-J. Surf. Sci. Nanotechnol. 4, 394 (2006)

P. Wurz, S.A. Fuselier, E. Möbius, H.O. Funsten, P.C. Brandt, F. Allegrini, A.G. Ghielmetti, R. Harper, E. Hertzberg, P. Janzen, H. Kucharek, D.J. McComas, E.C. Roelof, L. Saul, J.A. Scheer, M. Wieser, Y. Zheng, Space Sci. Rev. 146, 173 (2009)

G.P. Zank, Space Sci. Rev. 89, 413 (1999a)

G.P. Zank, AIP Conf. Proc. 471, 783 (1999b)

G.P. Zank, Annu. Rev. Astron. Astrophys. 53, 449 (2015)

G.P. Zank, Geosci. Lett. 3, 22 (2016)

G.P. Zank, P.C. Frisch, Astrophys. J. 518, 965 (1999). https://doi.org/10.1086/307320

G.P. Zank, H.L. Pauls, I.H. Cairns, G.M. Webb, J. Geophys. Res. 101, 457 (1996)

G.P. Zank, G. Li, O. Verkhoglyadova, Space Sci. Rev. 130, 255 (2007)

G.P. Zank, J. Heerikhuisen, B.E. Wood, N.V. Pogorelov, E. Zirnstein, D.J. McComas, Astrophys. J. 763, 20 (2013)

G.P. Zank, P. Hunana, P. Mostafavi, M.L. Goldstein, Astrophys. J. 797, 87 (2014)

G.P. Zank, S. Du, P. Hunana, Astrophys. J. 842, 114 (2017)

E.J. Zirnstein, J. Heerikhuisen, G.P. Zank, N.V. Pogorelov, D.J. McComas, M.I. Desai, Astrophys. J. 783, 129 (2014)

E.J. Zirnstein, J. Heerikhuisen, D.J. McComas, Astrophys. J. Lett. 804, L22 (2015a)

E.J. Zirnstein, J. Heerikhuisen, N.V. Pogorelov, D.J. McComas, M.A. Dayeh, Astrophys. J. 804, 5 (2015b)

E.J. Zirnstein, H.O. Funsten, J. Heerikhuisen, D.J. McComas, N.A. Schwadron, G.P. Zank, Astrophys. J. 826, 58 (2016a)

E.J. Zirnstein, J. Heerikhuisen, H.O. Funsten, G. Livadiotis, D.J. McComas, N.V. Pogorelov, Astrophys. J. Lett. 818, L18 (2016b)

E.J. Zirnstein, J. Heerikhuisen, G.P. Zank, N.V. Pogorelov, H.O. Funsten, D.J. McComas, D.B. Reisenfeld, N.A. Schwadron, Astrophys. J. 836, 238 (2017)

E.J. Zirnstein, J. Heerikhuisen, D.J. McComas, N.V. Pogorelov, D.B. Reisenfeld, J.R. Szalay, Astrophys. J. 859, 104 (2018a)

E.J. Zirnstein, R. Kumar, J. Heerikhuisen, D.J. McComas, A. Galli, Astrophys. J. 860, 170 (2018b)

E.J. Zirnstein, D.J. McComas, R. Kumar, H.A. Elliott, J.R. Szalay, C.B. Olkin, J. Spencer, S.A. Stern, L.A. Young, Phys. Rev. Lett. 121, 075102 (2018c). https://doi.org/10.1103/PhysRevLett.121.075102

R.D. Zwickl, K.A. Doggett, S. Sahm, W.P. Barrett, R.N. Grubb, T.R. Detman, V.J. Raben, C.W. Smith, P. Riley, R.E. Gold, R.A. Mewaldt, T. Maruyama, Space Sci. Rev. 86, 633 (1998) 